\title{
A paradigm for higher-order polygonal elements in finite elasticity using a gradient correction scheme
}

\author{
Heng Chi ${ }^{\mathrm{a}}$, Cameron Talischi ${ }^{\mathrm{b}}$, Oscar Lopez-Pamies ${ }^{\mathrm{b}}$, Glaucio H. Paulino ${ }^{\mathrm{a}}$ \\ ${ }^{a}$ School of Civil and Environmental Engineering, Georgia Institute of Technology, 790 Atlantic Drive, Atlanta, \\ GA, 30332, USA \\ ${ }^{b}$ Department of Civil and Environmental Engineering, University of Illinois at Urbana-Champaign, 205 North \\ Mathews Ave., Urbana, IL, 61801, USA
}

\begin{abstract}
Recent studies have demonstrated that polygonal elements possess great potential in the study of nonlinear elastic materials under finite deformations. On the one hand, these elements are well suited to model complex microstructures (e.g. particulate microstructures and microstructures involving different length scales) and incorporating periodic boundary conditions. On the other hand, polygonal elements are found to be more tolerant to large localized deformations than the standard finite elements, and to produce more accurate results in bending and shear than the standard finite elements. With mixed formulations, lower order mixed polygonal elements are also shown to be numerically stable on Voronoi-type meshes without any additional stabilization treatments. However, polygonal elements generally suffer from persistent consistency errors under mesh refinement with the commonly used numerical integration schemes. As a result, non-convergent finite element results typically occur, which severely limit their applications. In this work, a general gradient correction scheme is adopted that restores the polynomial consistency by adding a minimal perturbation to the gradient of the displacement field. With the correction scheme, the recovery of the optimal convergence for solutions of displacement-based and mixed formulations with both linear and quadratic displacement interpolants is confirmed by numerical studies of several boundary value problems in finite elasticity. In addition, for mixed polygonal elements, the various choices of the pressure field approximations are discussed, and their performance on stability and accuracy are numerically investigated. We present applications of those elements in physically-based examples including a study of filled elastomers with interphasial effect and a qualitative comparison with cavitation experiments for fiber reinforced elastomers.
\end{abstract}

Keywords: Gradient correction; finite elasticity; quadratic polygonal element; mixed variational principle; filled elastomers; cavitation

Email addresses: hchi6@gatech.edu (Heng Chi), talischi@gmail.com (Cameron Talischi), pamies@illinois.edu (Oscar Lopez-Pamies), paulino@gatech.edu (Glaucio H. Paulino) 


\section{Introduction}

The finite element space for polygonal elements contains non-polynomial (e.g. rational) functions and thereby the existing quadratures schemes, typically designed for integration of polynomial functions, will lead to persistent consistency errors that do not vanish with mesh refinement $[1,2]$. As a direct consequence, the so-called patch test, which provides a measure of polynomial consistency of conforming discretizations, is not passed on general polygonal meshes, even in an asymptotic sense. Moreover, the persistence of the errors in turn renders the finite element method suboptimally convergent or even non-convergent under mesh refinement. In practice, using sufficiently large number of integration points can lower the consistency error. For linear polygonal elements in two dimension (2D), a triangulation scheme with three integration points per triangle is shown to be sufficiently accurate for practical problems and mesh sizes $[1,3]$. However, for higher-order polygonal elements, for instance, quadratic elements that will be discussed in this paper, the number of integration points of such a scheme can become prohibitively large. This is also the case for polyhedral elements in three dimensions (3D). For example, maintaining optimal convergence rates with a linear polyhedral discretization for practical levels of mesh refinement may require several hundred integration points per element $[2,4]$. As the number of elements increase, the associated computational cost on a polyhedral discretization can be too expensive for practical applications.

Several attempts have been made in the literature to address this issue. For example, in the context of scalar diffusion problems, inspired by the virtual element method (VEM) $[5,6,7]$, Talischi et al. [1] has proposed a polynomial projection approach to ensure the polynomial consistency of the bilinear form, and thereby ensure the satisfaction of the patch test and optimal convergence for both linear and quadratic polygonal elements. A similar approach is also adopted by Manzini et al. [2] to solve Poisson problems on polyhedral meshes. However, those approaches require the existence of a bilinear form, and therefore extension to general nonlinear problem is non-trivial and is still an open question. Borrowing the idea of pseudo-derivatives in the meshless literature [8], Bishop has proposed an approach to correct the derivatives of the shape functions to enforce the linear consistency property on general

polygonal and polyhedral meshes $[9,10]$. With the correction, the linear patch test is passed and optimal convergence is achieved. Although being applicable for general nonlinear cases, extension to higher order cases(e.g. quadratic polygonal finite elements) is not trivially implied.

More recently, Talischi et al. [4] have proposed a general gradient correction scheme that is applicable to both linear and nonlinear problems on polygonal and polyhedral elements with arbitrary orders. In essence, the scheme corrects the gradient field at the element level with a minimal perturbation such that the discrete divergence theorem is satisfied against polynomial 
functions of suitable orders. With a minimum accuracy requirement of the numerical integration scheme, the correction scheme has been previously shown to restore optimal convergence for linear diffusion and nonlinear Forchheimer flow problems [4]. In this work, we adopt the gradient correction scheme in two dimensional finite elasticity problems and apply it to linear and quadratic polygonal elements. As we will see, the gradient correction scheme renders both linear and quadratic polygonal elements optimally convergent.

To enable the modeling of materials with a full range of compressibility, this work considers displacement-based as well as two-field mixed polygonal elements, the latter of which contains an additional discrete pressure field. For mixed finite elements, the numerical stability is a critical issue to ensure convergence and therefore has been subjected to extensive studies in the finite element literature. Generally, the stability condition is described by the well-known inf-sup condition $[11,12,13]$, which, in essence, implies a balance between the discrete spaces for displacement field and pressure field [14]. Many of the classical mixed finite elements are known to be unstable (see, for instance, summaries in $[15,16]$ ). As a result, some post-processing procedures or stabilization methods are needed for those elements (see, for instance, $[17,18,19,20])$. In contrast, some recent contributions have suggested that linear mixed polygonal elements coupled with element-wise constant pressure field are numerically stable on Voronoi-type meshes in both linear and nonlinear problems if every node/vertex in the mesh is connected to at most three edges $[14,21,3]$. Furthermore, with the availability of higher order displacement interpolants (see, e.g. [22, 23]), various mixed approximations for higher order polygonal elements featuring more enriched pressure spaces are made possible. For example, for quadratic mixed elements, together with quadratic interpolation of the displacement field, the pressure field can be approximated by either discontinuous piecewise-linear or continuous linearly complete functions. However, their stability, convergence and accuracy is still an open problem and has not been fully explored in the literature. In this paper, the performance of quadratic mixed polygonal elements with different choices of pressure approximations is presented and studied for the first time with thorough numerical assessments. As a direct observation, the quadratic mixed polygonal elements also seem to be stable with both discontinuous piecewise-linear and continuous linearly complete interpolations of the pressure field on Voronoi-type meshes for linear elasticity problems. Intuitively, this stability results from the larger displacement space for polygonal finite elements when compared with the classical triangular and quadrilateral elements.

The remainder of the paper is organized as follows. Displacement and mixed variational formulations of finite elasticity are briefly recalled in Section 2. In Section 3, finite element spaces and approximations are presented and numerical integration issues are discussed, including a review of the gradient correction scheme. Section 4 presents a numerical study of the convergence, accuracy, and stability for both linear and quadratic, displacement-based and 
mixed polygonal elements. In Section 5, two examples of practical relevance are studied with polygonal finite elements: $(i)$ the nonlinear elastic response of a filled elastomer reinforced with a random isotropic distribution of circular particles bonded through finite-size interphases, and (ii) the onset of cavitation in a fiber reinforced elastomer. Finally, some concluding remarks are recorded in Section 6.

We briefly and partially introduce the notation adopted in this paper. For any functional $f(x)$ that depends on field $x$, we write $\mathrm{D}^{x} f(x) \cdot y$ as the variation of $f(x)$ with respect to $x$, where $y$ is a trial field. Moreover, for any subset $E$ of the given domain $\Omega, E \subset \Omega$, we denote by $|E|$ its area or volume and $\langle\cdot\rangle_{E}$ the average operator:

$$
\langle\cdot\rangle_{E} \doteq \frac{1}{|E|} \int_{E}(\cdot) \mathrm{d} \mathbf{X}
$$

If the average is taken for the whole domain $\Omega$, we denote the operator as $\langle\cdot\rangle$ with the subscript $\Omega$ omitted. We shall also $\|\cdot\|_{\mathcal{L}^{2}(E)}$ to denote as the standard $\mathcal{L}^{2}$-norm over $E$ and $\|\cdot\|$ as the standard $\mathcal{L}^{2}$-norm over $\Omega$.

\section{Finite elasticity formulations}

In this section, for later use, two variational formulations of elastostatics are recalled, including the classical displacement-based formulation and a general two-field mixed formulation [24, 25, 26, 27]. Throughout, a Langranian description of the fields is adopted.

Consider a body in its stress-free, undeformed configuration that occupies a domain $\Omega$ with boundary $\partial \Omega$. On its boundary, it is subjected to a prescribed displacement field $\mathbf{u}^{0}$ on $\Gamma^{\mathbf{X}}$ and prescribed surface traction $\mathbf{t}$ (per unit undeformed surface) on $\Gamma^{\mathbf{t}}$, such that $\Gamma^{\mathbf{X}} \cup \Gamma^{\mathbf{t}}=\partial \Omega$ and $\Gamma^{\mathbf{X}} \cap \Gamma^{\mathbf{t}}=\varnothing$. Moreover, it is also assumed to be subjected to a body-force $\mathbf{f}$ (per unit undeformed volume) in $\Omega$. A stored-energy function $W$ is used to characterize the constitutive behavior of the body, which is assumed to be an objective function of the deformation gradient F. In terms of $W$, the first Piola-Kirchhoff stress tensor $\mathbf{P}$ at each material point $\mathbf{X} \in \Omega$ is given by the following relation:

$$
\mathbf{P}(\mathbf{X})=\frac{\partial W}{\partial \mathbf{F}}(\mathbf{X}, \mathbf{F}),
$$

which is used as the stress measure of choice in this paper.

\subsection{Displacement-based formulation}

The displacement-based formulation considers the displacement field $\mathbf{u}$ as the only independent field. The deformation gradient $\mathbf{F}$ is then assumed to be a function of $\mathbf{u}$ given by $\mathbf{F}(\mathbf{u})=\mathbf{I}+\nabla \mathbf{u}$, where $\nabla$ denotes the gradient operator with respect to the undeformed configuration and $\mathbf{I}$ is the identity in the space of second order tensors. According to the principle 
of minimum potential energy, the unknown equilibrium displacement field $\mathbf{u}$ is the one that minimizes the potential energy $\Pi$ among the set of all kinematically admissible displacements v:

$$
\Pi(\mathbf{u})=\min _{\mathbf{v} \in \mathcal{K}} \Pi(\mathbf{v})
$$

with

$$
\Pi(\mathbf{v})=\int_{\Omega} W(\mathbf{X}, \mathbf{F}(\mathbf{v})) \mathrm{d} \mathbf{X}-\int_{\Omega} \mathbf{f} \cdot \mathbf{v} \mathrm{d} \mathbf{X}-\int_{\Gamma^{\mathbf{t}}} \mathbf{t} \cdot \mathbf{v} \mathrm{d} S
$$

where $\mathcal{K}$ stands for the set of kinematically admissible displacements such that $\mathbf{v}=\mathbf{u}^{0}$ on $\Gamma^{\mathbf{X}}$.

The weak form of the Euler-Lagrange equations associated with the variational principle (3) is given by:

$G(\mathbf{u}, \mathbf{v})=\mathrm{D}^{\mathbf{u}} \Pi(\mathbf{u}) \cdot \mathbf{v}=\int_{\Omega} \frac{\partial W}{\partial \mathbf{F}}(\mathbf{X}, \mathbf{F}(\mathbf{u})): \nabla \mathbf{v d} \mathbf{X}-\int_{\Omega} \mathbf{f} \cdot \mathbf{v d} \mathbf{X}-\int_{\Gamma^{\mathbf{t}}} \mathbf{t} \cdot \mathbf{v} \mathrm{d} S=0 \quad \forall \mathbf{v} \in \mathcal{K}_{0}$,

where $\mathcal{K}_{0}$ denotes the set of all the kinematically admissible displacement fields that vanish on $\Gamma^{\mathbf{X}}$.

\subsection{A general two-field mixed variational formulation}

The displacement-based formulation is known to perform poorly with standard finite elements when the material under consideration is nearly or purely incompressible. Mixed variational principles are typically adopted as the formulation of choice for such problems instead. Recently, a new two-field mixed variational principle has been put forth by Chi et al. [3], which, unlike the commonly used mixed formulations in the finite element literature, does not require the multiplicative decomposition of the deformation gradient tensor $\mathbf{F}$ [3]. For the reminder of the paper, the formulation of Chi et al. [3] is referred to as the $\mathbf{F}$-formulation, whereas the commonly used formulation making use of the multiplicative decomposition of $\mathbf{F}$ into a deviatoric part, $\overline{\mathbf{F}}=(\operatorname{det} \mathbf{F})^{-\frac{1}{3}} \mathbf{F}$, and a hydrostatic part, $(\operatorname{det} \mathbf{F})^{\frac{1}{3}} \mathbf{I}$, is referred to as the $\overline{\mathbf{F}}$-formulation. In this section, the $\mathbf{F}$-formulation is briefly reviewed. For the $\overline{\mathbf{F}}$-formulation, the interested reader is referred to $[28,29,30,31,32,33,34,3]$ and references therein.

The $\mathbf{F}$-formulation consists of finding the equilibrium $(\mathbf{u}, \widehat{p}) \in \mathcal{K} \times \mathcal{Q}$, such that,

$$
\widehat{\Pi}(\mathbf{u}, \widehat{p})=\min _{\mathbf{v} \in \mathcal{K}} \max _{\widehat{q} \in \mathcal{Q}} \widehat{\Pi}(\mathbf{v}, \widehat{q})
$$

with

$$
\widehat{\Pi}(\mathbf{v}, \widehat{q})=\int_{\Omega}\left\{-\widehat{W}^{*}(\mathbf{X}, \mathbf{F}(\mathbf{v}), \widehat{q})+\widehat{q}[\operatorname{det} \mathbf{F}(\mathbf{v})-1]\right\} \mathrm{d} \mathbf{X}-\int_{\Omega} \mathbf{f} \cdot \mathbf{v d} \mathbf{X}-\int_{\Gamma^{\mathbf{t}}} \mathbf{t} \cdot \mathbf{v d} S
$$

In the above variational principle, $\mathcal{Q}$ denotes the set of square-integrable scalar functions and 
the "complementary" stored-energy function $\widehat{W}^{*}(\mathbf{X}, \mathbf{F}, \widehat{q})$ is defined by partial Legendre transformation:

$$
\widehat{W}^{*}(\mathbf{X}, \mathbf{F}, \widehat{q})=\max _{J}\{\widehat{q}(J-1)-\widehat{W}(\mathbf{X}, \mathbf{F}, J)\}
$$

where $\widehat{W}$ is defined such that $\widehat{W}(\mathbf{X}, \mathbf{F}, J)=W(\mathbf{X}, \mathbf{F})$ when $J=\operatorname{det} \mathbf{F}$.

Unlike the $\overline{\mathbf{F}}$-formulation, in which the second independent field agrees precisely with the Cauchy hydrostatic pressure field $p \doteq \operatorname{tr} \boldsymbol{\sigma}$ with $\boldsymbol{\sigma}=J^{-1} \mathbf{P F}^{T}$, the maximizing scalar field $\widehat{p}$ is a pressure-like field, henceforth referred to as the pressure field, relates to the hydrostatic pressure field $p$ via the relation

$$
p=\widehat{p}-\frac{1}{3 \operatorname{det} \mathbf{F}} \frac{\partial \widehat{W}^{*}}{\partial \mathbf{F}}(\mathbf{X}, \mathbf{F}, \widehat{p}): \mathbf{F} .
$$

The weak form of the Euler-Lagrange equations associated with (6)-(7) reads as

$$
\begin{array}{r}
G^{\mathbf{u}}(\mathbf{u}, \widehat{p}, \mathbf{v})=\mathrm{D}^{\mathbf{u}} \widehat{\Pi}(\mathbf{u}, \widehat{p}) \cdot \mathbf{v}=\int_{\Omega}\left[-\frac{\partial \widehat{W}^{*}}{\partial \mathbf{F}}(\mathbf{X}, \mathbf{F}(\mathbf{u}), \widehat{p})+\widehat{p} \operatorname{adj}(\mathbf{F}(\mathbf{u}))\right]: \nabla \mathbf{v d} \mathbf{X} \\
-\int_{\Omega} \mathbf{f} \cdot \mathbf{v d} \mathbf{X}-\int_{\Gamma^{\mathbf{t}}} \mathbf{t} \cdot \mathbf{v d} S=0 \quad \forall \mathbf{v} \in \mathcal{K}^{0}, \\
G^{\widehat{p}}(\mathbf{u}, \widehat{p}, \widehat{q})=\mathrm{D}^{\widehat{p}} \widehat{\Pi}(\mathbf{u}, \widehat{p}) \cdot \widehat{q}=\int_{\Omega}\left[\operatorname{det} \mathbf{F}(\mathbf{u})-1-\frac{\partial \widehat{W}^{*}}{\partial \widehat{p}}(\mathbf{X}, \mathbf{F}(\mathbf{u}), \widehat{p})\right] \widehat{q} \mathrm{~d} \mathbf{X}=0 \quad \forall \widehat{q} \in \mathcal{Q},
\end{array}
$$

where $\operatorname{adj}(\cdot)$ stands for the adjugate operator.

\section{Polygonal finite elements approximations}

This section addresses issues concerning polygonal finite element approximations. In particular, the constructions of conforming finite dimensional displacement space and pressure spaces, for both linear and quadratic polygonal finite elements, are presented. Furthermore, the gradient correction scheme of Talischi et al. [4] is reviewed and the numerical integration schemes used in this work are discussed. Finally, we record Galerkin approximations of the weak form of the Euler-Lagrange equations of the variational principles discussed in the preceding section including the incorporation of the gradient correction scheme for displacement-based and mixed formulations, and demonstrate the polynomial consistency of the proposed approximations.

\subsection{Finite element spaces}

Consider $\Omega_{h}$ to be a finite element decomposition of the domain $\Omega$ into non-overlapping polygons, where $h$ is the maximum element size. The boundary of the mesh, denoted as $\Gamma_{h}$ is assumed to be compatible with the applied boundary condition, that is, $\Gamma_{h}^{\mathbf{t}}$ and $\Gamma_{h}^{\mathbf{X}}$ are 
both unions of edges of the mesh. We also denote $E \in \Omega_{h}$ as the element of the mesh. The displacement space associated with the discretization is a conforming finite dimensional space $\mathcal{K}_{h, k}$ that defined as:

$$
\mathcal{K}_{h, k}=\left\{\mathbf{v}_{h} \in\left[C^{0}(\Omega)\right]^{2} \cap \mathcal{K}:\left.\mathbf{v}_{h}\right|_{E} \in\left[\mathcal{M}_{k}(E)\right]^{2}, \forall E \in \Omega_{h}\right\}
$$

where $k$ is the order of the discretization. In the above definition, $\mathcal{M}_{k}(E)$ is a finite dimensional space defined over each element $E$ whose basis functions are denoted as $\varphi_{i}^{k}$ henceforth. In this paper, we consider linear and serendipity quadratic elements, corresponding to the case with $k=1$ and $k=2$, respectively.

For a linear polygonal element $E$ with $n$ edges, the space $\mathcal{M}_{1}(E)$ is a $n$ dimensional space, with the degrees of freedom at each vertex of $E$, as shown in Fig. 1 (a), which can be defined by a set of generalized barycentric coordinates $\varphi_{i}^{1}$. Quite a few barycentric coordinates can be found in the literature [35, 36, 37, 38, 39, 40, 41, 42], among which the Mean Value coordinates [43] are adopted in this paper. Over element E, the Mean Value coordinate associated with

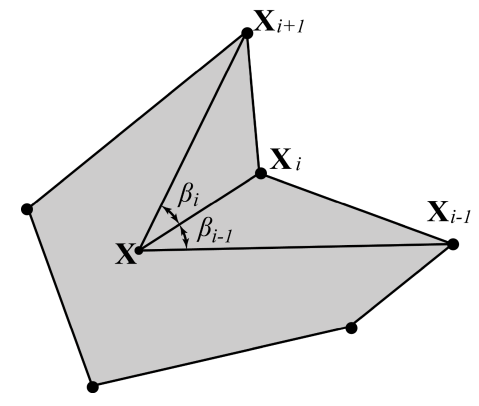

(a)

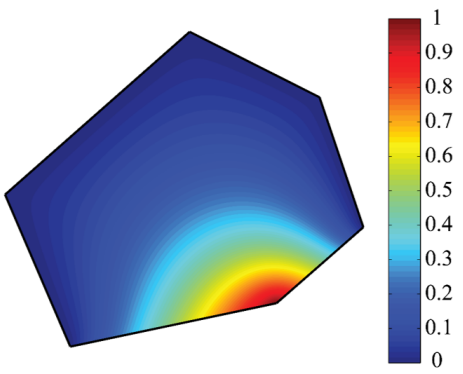

(b)

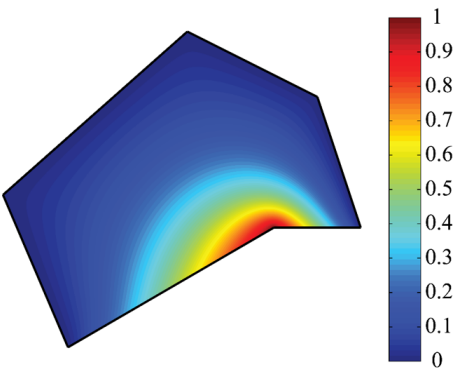

(c)

Figure 1: (a) Illustration of angles $\beta_{i}$ defined in Mean Value Coordinates interpolant $w_{i}$. (b) Contour plot of a Mean Value basis vector $\varphi_{i}^{1}$ over a convex polygon. (c) Contour plot of a Mean Value basis vector $\varphi_{i}^{1}$ over a concave polygon.

vertex $i$ is defined as [43]:

$$
\varphi_{i}^{1}(\mathbf{X})=\frac{w_{i}(\mathbf{X})}{\sum_{j=1}^{n} w_{j}(\mathbf{X})}
$$

with $w_{i}$ given by

$$
w_{i}(\mathbf{X})=\frac{\tan \left[\frac{\beta_{i-1}(\mathbf{X})}{2}\right]+\tan \left[\frac{\beta_{i}(\mathbf{X})}{2}\right]}{\left\|\mathbf{X}-\mathbf{X}_{i}\right\|},
$$

where $\mathbf{X}_{i}$ is the position vector of vertex $i$ and $t_{i}$ follows the definition $t_{i}=\tan \left(\beta_{i} / 2\right)$ in which $\beta_{i}$ is the angle defined in Fig. 1(a). By defining

$$
\mathbf{c}_{i}=\frac{\mathbf{X}_{i}-\mathbf{X}}{\left\|\mathbf{X}_{i}-\mathbf{X}\right\|^{2}}-\frac{\mathbf{X}_{i+1}-\mathbf{X}}{\left\|\mathbf{X}_{i+1}-\mathbf{X}\right\|^{2}},
$$


the ratio $\mathbf{R}_{i} \doteq \nabla w_{i} / w_{i}$ is expressed as

$$
\mathbf{R}_{i}=\left(\frac{t_{i-1}}{t_{i-1}+t_{i}}\right) \frac{\mathbf{c}_{i-1}^{\perp}}{\sin \beta_{i-1}}+\left(\frac{t_{i}}{t_{i-1}+t_{i}}\right) \frac{\mathbf{c}_{i}^{\perp}}{\sin \beta_{i}}+\frac{\mathbf{X}_{i}-\mathbf{X}}{\left\|\mathbf{X}_{i}-\mathbf{X}\right\|^{2}}
$$

In the above expression, we have made use of the notation $\mathbf{a}^{\perp}=\left\{-a_{y}, a_{x}\right\}$ to denote the $90^{\circ}$ counterclockwise rotation of a given vector $\mathbf{a}=\left\{a_{x}, a_{y}\right\} \in \mathbb{R}^{2}$, and $\|\mathbf{a}\|$ to denote the its norm. As a result, the gradients of the Mean Value coordinates are given by the following $[44,45]$ :

$$
\nabla \varphi_{i}^{1}=\varphi_{i}^{1}\left(\mathbf{R}_{i}-\sum_{j=1}^{n} \varphi_{j}^{1} \mathbf{R}_{j}\right)
$$

Unlike the Wachspress coordinates [44], the gradients of the Mean Value coordinates are shown to stay bounded as the interior angles approaching $\pi[46]$, meaning that the Mean Value coordinates are able to handle polygons with collinear vertices. Furthermore, the Mean Value coordinates are shown to be well defined for concave polygons [47]. Examples of contour plots of the Mean Value coordinates on a convex and concave polygons are shown in Fig. 1 (b) and (c).

For a serendipity quadratic elements $(k=2), \mathcal{M}_{2}(E)$ is a $2 n$ dimensional space having the degrees of freedom at the each vertex of $E$, as well as the mid-point of each edge, as shown in Figs. 2 (a) and (d). According to Rand et al [22], such a space can be constructed from linear combination of pairwise products of the barycentric coordinates $\varphi_{i}^{1}$. Its interpolants $\varphi_{i}^{2}$ are expressed as:

$$
\varphi_{i}^{2}(\mathbf{X})=\sum_{j=1}^{n} \sum_{l=1}^{n} c_{j l}^{i} \varphi_{j}^{1}(\mathbf{X}) \varphi_{l}^{1}(\mathbf{X}), \quad i=1, \cdots, 2 n .
$$

Here, $\varphi_{j}^{1}$ are the barycentric coordinates for $E$, which are the Mean Value coordinates in this work. The coefficients $c_{j l}^{i}$ are computed such that any quadratic functions can be interpolated exactly by $\varphi_{i}^{2}$ :

$$
p(\mathbf{X})=\sum_{i=1}^{n}\left[p\left(\mathbf{X}_{i}\right) \varphi_{i}^{2}(\mathbf{X})+p\left(\widehat{\mathbf{X}}_{i}\right) \varphi_{i+n}^{2}(\mathbf{X})\right], \quad \forall p \in \mathcal{P}_{2}(E)
$$

where, $\widehat{\mathbf{X}}_{i}=\left(\mathbf{X}_{i}+\mathbf{X}_{i+1}\right) / 2$, are the positions of the mid-side nodes. By definition, the coefficients $c_{j l}^{i}$ depend only on the coordinates of the vertices of $E$. Therefore, the gradients of the interpolants $\varphi_{i}^{2}$ are obtained as:

$$
\nabla \varphi_{i}^{2}=\sum_{j=1}^{n} \sum_{l=1}^{n}\left(c_{j l}^{i}+c_{l j}^{i}\right) \nabla \varphi_{j}^{1}(\mathbf{X}) \varphi_{l}^{1}(\mathbf{X})
$$


Although the construction above is derived assuming that the polygonal elements are strictly convex [22], we find it also seems to be valid for several cases of concave polygons provided that the barycentric coordinates $\varphi_{j}^{1}$ in Eq.(18) are well defined over concave polygons, which holds for Mean Value coordinates. In fact, our numerical assessments in Section 4 suggest that the finite element solutions with certain non-convex polygonal elements indeed converge with their optimal rates. Examples of the basis function constructed using this approach is shown in Figs. 2 (b), (c), (e) and (f) on both convex and concave polygons.

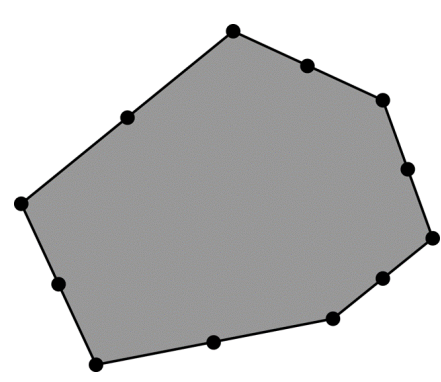

(a)

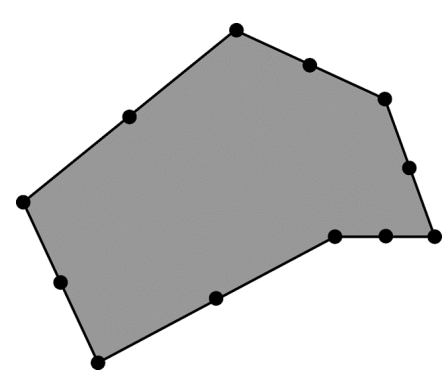

(d)

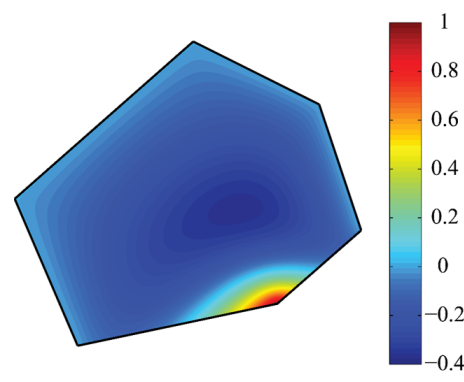

(b)

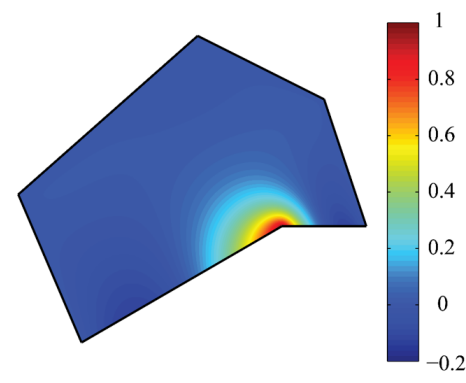

(e)

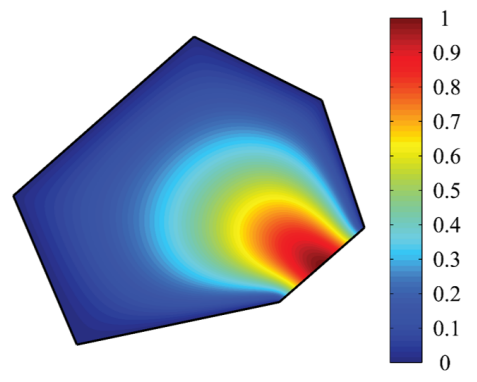

(c)

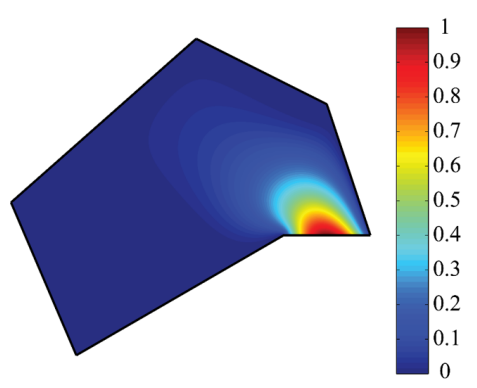

(f)

Figure 2: (a) Illustration of the vertex and mid-edge degrees of freedom of a convex polygon. (b) Contour plot of a Mean Value basis $\varphi_{i}^{2}$ associated with a vertex over a convex polygon. (c) Contour plot of a Mean Value basis $\varphi_{i}^{2}$ associated with a mid-edge node over a convex polygon.(d) Illustration of the vertex and mid-edge degrees of freedom of a concave polygon. (e) Contour plot of a Mean Value basis $\varphi_{i}^{2}$ associated with a vertex over a concave polygon. (f) Contour plot of a Mean Value basis $\varphi_{i}^{2}$ associated with a mid-edge node over a concave polygon.

Following their definitions, the spaces $\mathcal{M}_{1}(E)$ and $\mathcal{M}_{2}(E)$ contain all the polynomial functions of order $k$ over $E$, namely,

$$
\mathcal{P}_{k}(E) \subseteq \mathcal{M}_{k}(E), \quad \forall E \in \Omega_{h}, k=1,2,
$$

where $\mathcal{P}_{k}(E)$ is the space of polynomial functions of order $k$. Their basis functions satisfy the Kronecker-delta property, that is $\varphi_{i}^{k}\left(\mathbf{X}_{j}\right)=\delta_{i j}$ with $k=1,2$. In addition, any functions in $\mathcal{M}_{k}(E)$ posses $k$ th order polynomial variations on $\partial E$.

Regarding the two-field mixed finite elements, approximation of the additional pressure field is needed. As conforming approximations of $\mathcal{Q}$, either discontinuous or continuous approxima- 
tions can be adopted. For discontinuous approximations, the discrete pressure space $\mathcal{Q}_{h, k-1}^{D}$ can be defined as:

$$
\mathcal{Q}_{h, k-1}^{D}=\left\{q_{h} \in \mathcal{Q}:\left.q_{h}\right|_{E} \in \mathcal{P}_{k-1}(E), \forall E \in \Omega_{h}\right\}
$$

where $k$ is the order of the element. As implied by the above definition, the approximated pressure field may be discontinuous across element boundaries. This type of mixed elements is similar to the Crouzeix-Raviart (C-R) elements in fluid problems[48]. For the remainder of the paper, we denote this family of mixed element as $\mathcal{M}_{k}-\mathcal{P}_{k-1}^{D}$ elements, where " $\mathcal{M}$ " denotes the Barycentric Mean Value spaces, " $\mathcal{P}^{D}$ " stands for polynomial spaces which are discontinuous across element boundaries, and $k$ is the order of the element. For instance, the pressure space of the $\mathcal{M}_{1}-\mathcal{P}_{0}^{D}$ element consists of piecewise constant functions, which are constant over each element. Similarly, the pressure space of the $\mathcal{M}_{2}-\mathcal{P}_{1}^{D}$ element contains piecewise linear functions that vary linearly over each element.

Alternatively, a continuous approximation of the pressure space can be defined in the following manner:

$$
\mathcal{Q}_{h, k-1}^{C}=\left\{q_{h} \in C^{0}(\Omega):\left.q_{h}\right|_{E} \in \mathcal{M}_{k-1}(E), \forall E \in \Omega_{h}\right\}
$$

This class of elements resembles the Taylor-Hood (T-H) elements in fluid problems[49] and they are denoted as the $\mathcal{M}_{k}-\mathcal{M}_{k-1}$ elements for the remainder of the paper. For convention, we define $\mathcal{M}_{0}=\mathcal{P}_{0}$. As a result, the $\mathcal{M}_{k}-\mathcal{M}_{k-1}$ elements coincide with the the $\mathcal{M}_{k}-\mathcal{P}_{k-1}^{D}$ elements for the linear case, i.e. $k=1$.

In this paper, we will consider both types of mixed polygonal finite elements up to quadratic order. As an illustration, the degrees of freedom (DOFs) of the displacement field and pressure field for those mixed polygonal elements are shown in Fig. 3.

\subsection{Numerical integration and gradient correction scheme}

Because of the non-polynomial nature of the space defined by the barycentric coordinates, commonly used quadrature rules for polynomial functions will introduce consistency errors that are persistent with mesh refinement and lead to non-convergent results in finite elasticity problem. Although higher order quadrature rules can reduce the consistency error, they may contain a large amount of integration points and consequently make it computationally expensive to iteratively evaluate stiffness and internal force vectors. To overcome the above-mentioned issues, we introduce the gradient correction scheme in this work to polygonal elements in the context of finite elasticity problems [4]. We note that although the gradient correction theory is applicable to both two dimensional (2D) and three dimensional (3D) problems, the following discussion is restricted to the $2 \mathrm{D}$ case.

Consider a general polygonal element $E$ with $\partial E$ denoted as its boundaries and $h_{E}$ as its diameter. As defined in the previous subsection, $\mathcal{M}_{k}(E)$ and $\mathcal{P}_{k}(E)$ are the finite element 


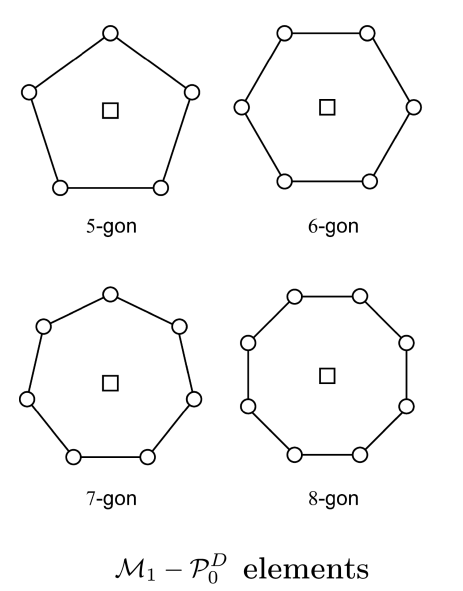

(a)

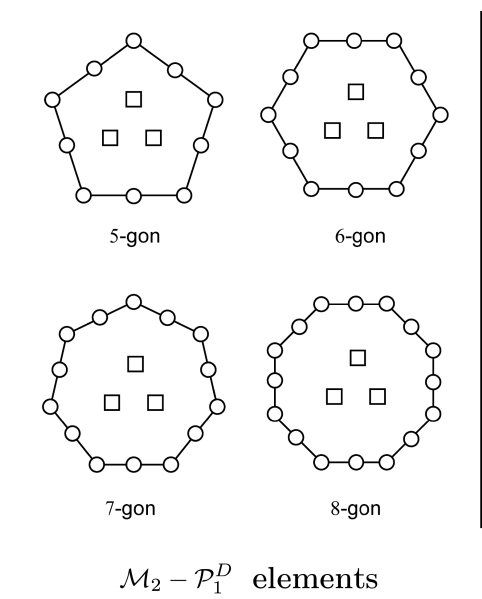

(b)

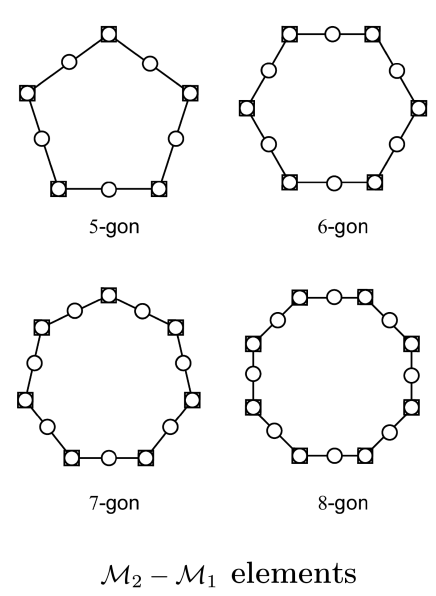

(c)

\section{Displacement $\square$ Pressure}

Figure 3: Illustration of the degrees of freedom of the displacement field and pressure field for different mixed polygonal element studied in this paper : (a) $\mathcal{M}_{1}-\mathcal{P}_{0}^{D}$ elements, (b) $\mathcal{M}_{2}-\mathcal{P}_{1}^{D}$ elements, and (c) $\mathcal{M}_{2}-\mathcal{M}_{1}$ elements.

space and polynomial space defined over E,respectively, which are of order $k$. In addition, we denote numerical integration schemes, $\oint_{E}$, on $E$ as an approximations of the area integral, $\int_{E}$. For the remainder of the paper, the integration scheme is referred to as $m$ th order if it can integrate polynomial functions of order $m$ exactly. Regarding the boundary integral, one dimensional Gauss-Lobatto quadrature rule is adopted for the line integral $\int_{\partial E}$, which uses two integration points per edge for linear elements and three integration points per edge for quadratic elements.

Accuracy requirements on numerical integrations. A minimum accuracy requirement is assumed for the candidate numerical integration schemes of $\oint_{E}[4]$. For a fixed element order $k$, hence the fixed order of space $\mathcal{M}_{k}(E)$ and $\mathcal{P}_{k}(E)$, the gradient correction scheme requires the available integration scheme $\oint_{E}$ to be exact when integrating any polynomial functions of order at least $2 k-2$. For instance, the selected scheme should integrate any constants (order 0 ) and quadratic (order 2) functions exactly for linear elements $(k=1)$ and quadratic elements $(k=2)$, respectively. Moreover, the integrations scheme $\oint_{E}$ needs to be sufficiently rich enough to eliminate spurious energy modes. One example of such a scheme is the triangulation scheme $[50,21]$. It divides each polygonal element into triangles by connecting the centroid to each vertex and applies available polynomially precise quadrature rules in each triangle. In this paper, a triangulation scheme with the Dunavant rules [51] in each subdivided triangle is adopted for both linear and quadratic elements. According to the above stated requirements, instead of using a one point rule that exactly integrates constant functions, the 1st order triangulation scheme containing one integration per subdivided triangle is used for linear element to avoid 
spurious energy modes. For a quadratic element, the 2nd order triangulation scheme is employed, which contains three integration points per subdivided triangle. Furthermore, the 3rd order triangulation scheme is also used to investigate the effect of increasing integration orders. Illustrations of those schemes are shown in Figs. 4 (a)-(c). As a side note, the triangulation scheme requires polygonal elements be star shaped with respect to their centroids, which is the case for all the examples presented in this paper. However, since the gradient correction scheme is also applicable to other quadrature schemes, as long as they satisfy the accuracy requirement stated in the paper, other more advance quadrature schemes available in the literature, e.g., $[52,53,54]$, which are specifically designed for integrating polynomial functions over arbitrary polygonal domains, can also be used as $\oint_{E}$.

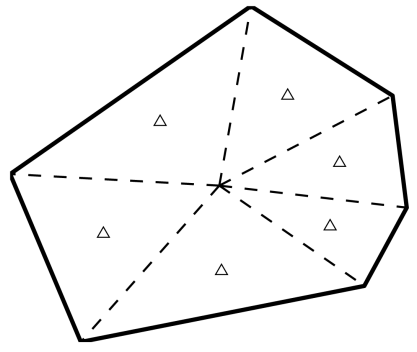

(a)

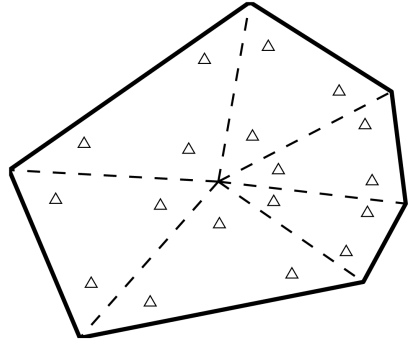

(b)

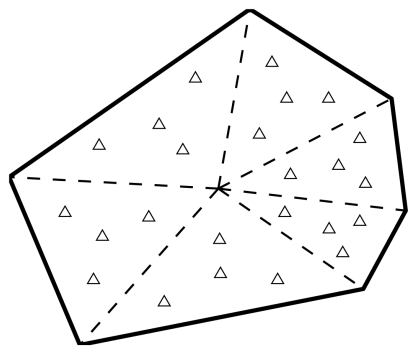

(c)

Figure 4: Illustration of the "triangulation" schemes for general polygons in physical domain: (a) 1st order triangulation scheme, (b) 2nd order triangulation scheme and (c) 3rd order triangulation scheme.

Under the above stated accuracy requirement, the gradient correction scheme corrects the exact gradient field by adding a small perturbation field to enforce the satisfaction of of the discrete divergence theorem at the element level. In the sequel, we first define the gradient correction scheme for scalar problems, and then show its extension to vector problems.

Gradient correction for scalar problems. For scalar problems, the corrected gradient, denoted as $\nabla_{E, k} v=\left\{\left(\nabla_{E, k} v\right)_{x},\left(\nabla_{E, k} v\right)_{y}\right\}^{T}$, is taken to be closest vector field to $\nabla v=\left\{(\nabla v)_{x},(\nabla v)_{y}\right\}^{T}$ that solves the following optimization problem:

$$
\min _{\boldsymbol{\zeta}} \oint_{E}(\boldsymbol{\zeta}-\nabla v) \cdot(\boldsymbol{\zeta}-\nabla v) \mathrm{d} \mathbf{X}
$$

subject to

$$
\oint_{E} \mathbf{p} \cdot \boldsymbol{\zeta} \mathrm{d} \mathbf{X}=\int_{\partial E}(\mathbf{p} \cdot \mathbf{N}) v \mathrm{~d} S-\oint_{E} v \operatorname{Divpd} \mathbf{X}, \quad \forall \mathbf{p} \in\left[\mathcal{P}_{k-1}(E)\right]^{2}
$$

The above minimization is performed over all the sufficiently smooth functions such that the utilized quadrature makes sense. Since quadrature is used, we note that the above minimization problem only determines $\nabla_{E, k} v$ at the quadrature points and the following analysis shows that 
the difference of $\nabla_{E, k} v-\nabla v$ equal to an element of $\left[\mathcal{P}_{k-1}(E)\right]^{2}$ at those points. Consider a basis of $\left[\mathcal{P}_{k-1}(E)\right]^{2}$ denoted as $\left\{\boldsymbol{\xi}_{1}, \cdots, \boldsymbol{\xi}_{n \mathcal{P}_{k-1}}\right\}$ where $n \mathcal{P}_{k-1}$ is the dimension $\left[\mathcal{P}_{k-1}(E)\right]^{2}$. We replace the constraint (25) with an equivalent set of constraints:

$$
\oint_{E} \boldsymbol{\xi}_{a} \cdot \boldsymbol{\zeta} \mathrm{d} \mathbf{X}=\int_{\partial E}(\boldsymbol{\xi} \cdot \mathbf{N}) v \mathrm{~d} S-\oint_{E} v \operatorname{Div} \boldsymbol{\xi} \mathrm{d} \mathbf{X}, \quad a=1, \cdots, n \mathcal{P}_{k-1} .
$$

Introduce a set of Lagrange multipliers, $\lambda_{1}, \cdots, \lambda_{n \mathcal{P}_{k-1}}$, the Lagrangian of the constrained optimization problem (24)-(25) takes the form

$$
\begin{aligned}
\mathcal{L}\left(\boldsymbol{\zeta}, \lambda_{1}, \cdots, \lambda_{n \mathcal{P}_{k-1}}\right)= & \oint_{E}(\boldsymbol{\zeta}-\nabla v) \cdot(\boldsymbol{\zeta}-\nabla v) \mathrm{d} \mathbf{X} \\
& +\sum_{a=1}^{n \mathcal{P}_{k-1}} \lambda_{a}\left[\oint_{E} \boldsymbol{\xi}_{a} \cdot \boldsymbol{\zeta} \mathrm{d} \mathbf{X}-\int_{\partial E}\left(\boldsymbol{\xi}_{a} \cdot \mathbf{N}\right) v \mathrm{~d} S+\oint_{E} v \operatorname{Div} \boldsymbol{\xi}_{a} \mathrm{~d} \mathbf{X}\right]
\end{aligned}
$$

Taking variation of the Lagrangian with respect to $\boldsymbol{\eta}$, the optimality condition of $\nabla_{E, k} v$ gives

$$
D^{\zeta} \mathcal{L}\left(\nabla_{E, k} v, \lambda_{1}, \cdots, \lambda_{n \mathcal{P}_{k-1}}\right) \cdot \boldsymbol{\eta}=\oint_{E}\left(\nabla_{E, k} v-\nabla v+\sum_{a=1}^{n \mathcal{P}_{k-1}} \frac{1}{2} \lambda_{a} \mathbf{p}_{a}\right) \cdot \boldsymbol{\eta}=0 .
$$

Therefore, motivated by above analysis, we formally defined $\nabla_{E, k} v$ as the vector field that satisfies the following two conditions:

$$
\begin{gathered}
\nabla_{E, k} v-\nabla v \in\left[\mathcal{P}_{k-1}(E)\right]^{2}, \text { and } \\
\oint_{E} \mathbf{p} \cdot \nabla_{E, k} v \mathrm{~d} \mathbf{X}=\int_{\partial E}(\mathbf{p} \cdot \mathbf{N}) v \mathrm{~d} S-\oint_{E} v \operatorname{Divpd} \mathbf{X}, \quad \forall \mathbf{p} \in\left[\mathcal{P}_{k-1}(E)\right]^{2} .
\end{gathered}
$$

Furthermore, notice that relation (30) holds for any functions in $\mathcal{P}_{k}(E)$. In such cases, as implied by the minimization problem (24) and (25), the correction function is zero and the corrected gradients coincide with the exact ones, implying

$$
\nabla_{E, k} q=\nabla q, \quad \forall q \in \mathcal{P}_{k}(E)
$$

By definition, we are able show that for any sufficiently smooth vector fields $\boldsymbol{\psi}$, the elementlevel consistency error satisfies the following estimate

$$
\oint_{E} \boldsymbol{\psi} \cdot \nabla_{E, k} v \mathrm{~d} \mathbf{X}-\int_{E} \boldsymbol{\psi} \cdot \nabla v \mathrm{~d} \mathbf{X}=\mathcal{O}\left(h_{E}^{k}\right)\|\nabla v\|_{\mathcal{L}^{2}(E)},
$$


where $h_{E}$ is the diameter of $E[4]$.

From a computational perspective, since $\nabla_{E, k}$ is a linear map, only the gradient of each basis function in $\mathcal{M}_{k}(E)$ needs to be corrected in practice. Here, we present a procedure for computing the corrected gradient of each basis function. We denote $\left\{\varphi_{1}^{k}, \cdots, \varphi_{n \mathcal{M}_{k}}^{k}\right\}$ as the basis for $\mathcal{M}_{k}(E)$, where $n \mathcal{M}_{k}$ is the dimensions of the space $\mathcal{M}_{k}(E)$. According to (29), we can find a coefficient matrix $\mathbf{S}$ such that

$$
\nabla_{E, k} \varphi_{i}^{k}=\nabla \varphi_{i}^{k}+\sum_{a=1}^{n \mathcal{P}_{k-1}} S_{i a} \boldsymbol{\xi}_{a}, \quad \forall i=1, \ldots, n \mathcal{M}_{k}
$$

We further define matrices $\mathbf{R}$ of size $n \mathcal{M}_{k} \times n \mathcal{P}_{k-1}$, and $\mathbf{M}$ of size $n \mathcal{P}_{k-1} \times n \mathcal{P}_{k-1}$ with the following forms:

$$
\begin{gathered}
R_{i a}=\int_{\partial E}\left(\boldsymbol{\xi}_{a} \cdot \mathbf{N}\right) \varphi_{i}^{k} \mathrm{~d} S-\oint_{E} \varphi_{i}^{k} \operatorname{Div}\left[\boldsymbol{\xi}_{a}\right] \mathrm{d} \mathbf{X}-\oint_{E} \boldsymbol{\xi}_{a} \cdot \nabla \varphi_{i}^{k} \mathrm{~d} \mathbf{X}, \text { and } \\
M_{a b}=\oint_{\partial E} \boldsymbol{\xi}_{a} \cdot \boldsymbol{\xi}_{b} \mathrm{~d} \mathbf{X} .
\end{gathered}
$$

Replacing $v$ and $\mathbf{p}$ with $\varphi_{i}^{k}$ and $\boldsymbol{\xi}_{b}$ in (30) yields the following linear system of equations

$$
\sum_{a=1}^{n \mathcal{P}_{k-1}} S_{i a} M_{a b}=R_{i b}, \quad \forall i=1, \ldots, n \mathcal{M}_{k} \text { and } b=1, \ldots, n \mathcal{P}_{k-1}
$$

Therefore, the coefficient matrix is obtained as $\mathbf{S}=\mathbf{R} \mathbf{M}^{-1}$.

Gradient correction for vectorial problems. When extended to vector field $\mathbf{v}=\left\{v_{x}, v_{y}\right\}^{T} \in$ $\left[\mathcal{M}_{k}(E)\right]^{2}$, the gradient correction scheme takes the form:

$$
\nabla_{E, k} \otimes \mathbf{v}=\left[\begin{array}{l}
\left(\nabla_{E, k} v_{x}\right)^{T} \\
\left(\nabla_{E, k} v_{y}\right)^{T}
\end{array}\right]
$$

Similar to the scalar case, the corrected gradient satisfies the discrete divergence theorem,

$$
\oint_{E} \mathbf{p}: \nabla_{E, k} \otimes \mathbf{v d} \mathbf{X}=\int_{\partial E}(\mathbf{p N}) \cdot \mathbf{v} \mathrm{d} S-\oint_{E} \mathbf{v} \cdot \operatorname{Div} \mathbf{p d} \mathbf{X}, \quad \forall \mathbf{p} \in\left[\mathcal{P}_{k-1}(E)\right]^{2 \times 2},
$$

and, moreover, for any sufficiently smooth 2nd order tensorial fields $\boldsymbol{\psi}$, the element-level consistency error satisfies

$$
\oint_{E} \boldsymbol{\psi}: \nabla_{E, k} \otimes \mathbf{v d} \mathbf{X}-\int_{E} \boldsymbol{\psi}: \nabla \mathbf{v d} \mathbf{X}=\mathcal{O}\left(h_{E}^{k}\right)\|\nabla \mathbf{v}\|_{\mathcal{L}^{2}(E)}
$$


In computational implementation, assuming the set of basis functions $\left\{\boldsymbol{\varphi}_{1}^{k}, \ldots, \boldsymbol{\varphi}_{2 n \mathcal{M}_{k}}^{k}\right\}$ of $\left[\mathcal{M}_{k}(E)\right]^{2}$ are of the form

$$
\varphi_{2 i-1}^{k}=\left\{\varphi_{i}^{k}, 0\right\}^{T}, \quad \varphi_{2 i}^{k}=\left\{0, \varphi_{i}^{k}\right\}^{T}, \quad i=1, \ldots, n \mathcal{M}_{k}
$$

the correction scheme for vectorial problems in practice amounts to correcting each basis functions as follows

$$
\nabla_{E, k} \otimes \boldsymbol{\varphi}_{2 i-1}^{k}=\left[\begin{array}{c}
\left(\nabla_{E, k} \varphi_{i}^{k}\right)^{T} \\
\mathbf{0}
\end{array}\right], \quad \nabla_{E, k} \otimes \boldsymbol{\varphi}_{2 i}^{k}=\left[\begin{array}{c}
\mathbf{0} \\
\left(\nabla_{E, k} \varphi_{i}^{k}\right)^{T}
\end{array}\right] \quad i=1, \ldots, n \mathcal{M}_{k}
$$

where $\nabla_{E, k} \varphi_{i}^{k}$ is computed according to above-mentioned procedure described for scalar problems.

\subsection{Conforming Galerkin approximations}

Consider the given discretization $\Omega_{h}$ of the domain and $\Gamma_{h}$ of its boundary, we define the numerical integration $\mathscr{S}_{\Omega_{h}}$ on $\Omega_{h}$, as the summation of the contributions from numerical integrals $\oint_{E}$ from element levels following typical assembly rules, namely, $\oiint_{\Omega_{h}}=\sum_{E \in \Omega_{h}} \oint_{E}$, and $\int_{\Gamma_{h}^{t}}$ as the numerical integration on $\Gamma_{h}^{\mathbf{t}}$ based on Gauss-Lobatto rule. In the same fashion, we define the discrete gradient map on the global level, $\nabla_{h, k}: \mathcal{K}_{h, k} \rightarrow\left[\mathcal{L}^{2}\left(\Omega_{h}\right)\right]^{2 \times 2}$, such that it coincides with gradient correction map $\nabla_{E, k}$ at the element level,

$$
\left.\left(\nabla_{h, k} \mathbf{v}_{h}\right)\right|_{E}=\nabla_{E, k} \otimes\left(\left.\mathbf{v}_{h}\right|_{E}\right), \quad \forall E \in \Omega_{h} \text { and } \mathbf{v}_{h} \in \mathcal{K}_{h, k}
$$

The Galerkin approximation of the displacement-based formulation consists of finding $\mathbf{u}_{h} \in$ $\mathcal{K}_{h, k}$, such that,

$$
G_{h}\left(\mathbf{u}_{h}, \mathbf{v}_{h}\right)=0, \quad \forall \mathbf{v}_{h} \in \mathcal{K}_{h, k}^{0}
$$

where $\mathcal{K}_{h, k}^{0}=\mathcal{K}_{h, k} \bigcap \mathcal{K}^{0}$ and $G_{h}\left(\mathbf{u}_{h}, \mathbf{v}_{h}\right)$ is the quadrature evaluation of $G(\mathbf{u}, \mathbf{v})$ in Equation (5) with the exact gradient operator $\nabla$ replaced by $\nabla_{h, k}$, which takes the form:

$$
G_{h}\left(\mathbf{u}_{h}, \mathbf{v}_{h}\right)=\oint_{\Omega_{h}} \frac{\partial W}{\partial \mathbf{F}}\left(\mathbf{X}, \mathbf{I}+\nabla_{h, k} \mathbf{u}_{h}\right): \nabla_{h, k} \mathbf{v}_{h} \mathrm{~d} \mathbf{X}-\oint_{\Omega_{h}} \mathbf{f}_{h} \cdot \mathbf{v}_{h} \mathrm{~d} \mathbf{X}-\int_{\Gamma_{h}^{\mathrm{t}}} \mathbf{t}_{h} \cdot \mathbf{v}_{h} \mathrm{~d} S
$$

and terms $\mathbf{f}_{h}$ and $\mathbf{t}_{h}$ are the approximated body force and boundary traction.

For the two-field mixed formulation, by introducing the additional finite element space $\mathcal{Q}_{h, k-1}^{D}\left(\right.$ or $\left.\mathcal{Q}_{h, k-1}^{C}\right) \subseteq \mathcal{Q}$, the Gakerkin approximation consist of finding $\left(\mathbf{u}_{h}, \widehat{p}_{h}\right) \in \mathcal{K}_{h, k} \times \mathcal{Q}_{h, k-1}^{D}($ or $\mathcal{Q}_{h, k-1}^{C}$, such that

$$
\begin{gathered}
G_{h}^{\mathbf{u}}\left(\mathbf{u}_{h}, \widehat{p}_{h}, \mathbf{v}_{h}\right)=0 \quad \forall \mathbf{v}_{h} \in \mathcal{K}_{h, k}^{0}, \\
G_{h}^{\widehat{p}}\left(\mathbf{u}_{h}, \widehat{p}_{h}, \widehat{q}_{h}\right)=0 \quad \forall \widehat{q}_{h} \in \mathcal{Q}_{h, k-1}^{D}\left(\text { or } \mathcal{Q}_{h, k-1}^{C}\right) .
\end{gathered}
$$


with $G_{h}^{\mathbf{u}}\left(\mathbf{u}_{h}, \widehat{p}_{h}, \mathbf{v}_{h}\right)$ and $G_{h}^{\widehat{p}}\left(\mathbf{u}_{h}, \widehat{p}_{h}, \widehat{q}_{h}\right)$ being of the form

$$
\begin{array}{r}
G_{h}^{\mathbf{u}}\left(\mathbf{u}_{h}, \widehat{p}_{h}, \mathbf{v}_{h}\right)=\oint_{\Omega_{h}}\left[-\frac{\partial \widehat{W}^{*}}{\partial \mathbf{F}}\left(\mathbf{X}, \mathbf{I}+\nabla_{h, k} \mathbf{u}_{h}, \widehat{p}_{h}\right)+\widehat{p}_{h} \operatorname{adj}\left(\mathbf{I}+\nabla_{h, k} \mathbf{u}_{h}\right)\right]: \nabla_{h, k} \mathbf{v}_{h} \mathrm{~d} \mathbf{X} \\
-\oint_{\Omega_{h}} \mathbf{f}_{h} \cdot \mathbf{v}_{h} \mathrm{~d} \mathbf{X}-\int_{\Gamma_{h}^{t}} \mathbf{t}_{h} \cdot \mathbf{v}_{h} \mathrm{~d} S, \\
G_{h}^{\widehat{p}}\left(\mathbf{u}_{h}, \widehat{p}_{h}, \widehat{q}_{h}\right)=\oint_{\Omega_{h}}\left[\operatorname{det}\left(\mathbf{I}+\nabla_{h, k} \mathbf{u}_{h}\right)-1-\frac{\partial \widehat{W}^{*}}{\partial \widehat{p}}\left(\mathbf{X}, \mathbf{I}+\nabla_{h, k} \mathbf{u}_{h}, \widehat{p}_{h}\right)\right] \widehat{q}_{h} \mathrm{~d} \mathbf{X} .
\end{array}
$$

Notice that since we replace the gradient operators $\nabla$ of both $\mathbf{u}_{h}$ and $\mathbf{v}_{h}$ in Eqs. (44), (47) and (48) with $\nabla_{h, k}$, the resulting approximations yield symmetric linearizations.

We finalize this section with several remarks regarding the performance of the above approximations in patch tests, which are typically adopted to assess the level of consistency error. In the discussions that follow, we restrict our attention to case where the descritization exactly represents the domain and boundary conditions, namely, $\Omega_{h}=\Omega, \Gamma^{\mathbf{t}}=\Gamma_{h}^{\mathbf{t}}$ and $\Gamma^{\mathbf{X}}=\Gamma_{h}^{\mathbf{X}}$. As a result, the errors arising from the approximation of the geometry is neglected in the following discussions.

First, we consider the first order patch test, in which the exact displacement field is a linear vector field, that is, $\mathbf{u}=\mathbf{p}_{1} \in\left[\mathcal{P}_{1}(\Omega)\right]^{2}$. We note that by the polynomial completeness property of the element-level space $\mathcal{M}_{1}(E)$, the exact displacement field is also in $\mathcal{K}_{h, 1}$. Accordingly, with any given stored-energy function $W(\mathbf{X}, \mathbf{F})$, the body force $\mathbf{f}$ is zero everywhere in $\Omega$ and the boundary traction $\mathbf{t}$ on $\Gamma^{\mathbf{t}}$ is given by $\mathbf{t}=\mathbf{P N}$, where $\mathbf{P}=\partial W\left(\mathbf{X}, \mathbf{F}\left(\mathbf{p}_{1}\right)\right) / \partial \mathbf{F}$. In addition, the associated exact pressure field is found as as

$$
\widehat{p}_{0}=\left\{\begin{array}{ll}
\text { constant } & \operatorname{det} \mathbf{F}\left(\mathbf{p}_{1}\right)=1 \\
\left.\frac{\partial \widehat{W}\left(\mathbf{X}, \mathbf{F}\left(\mathbf{p}_{1}\right), J\right)}{\partial J}\right|_{J=\operatorname{det} \mathbf{F}\left(\mathbf{p}_{1}\right)} & \text { otherwise }
\end{array},\right.
$$

where the constant pressure for incompressible solids is determined by applied boundary traction $\mathbf{t}$. We proceed to verify the exact passage of first order patch test by showing that $\mathbf{u}_{h}=\mathbf{p}_{1}$ and $\widehat{p}_{h}=\widehat{p}_{0}$ are solutions to Eqs. (43), (45) and (46). For displacement-based approximation, upon recognizing

$$
\nabla_{h, k} \mathbf{u}_{h}=\nabla_{h, k} \mathbf{p}_{1}=\nabla \mathbf{p}_{1} \in\left[\mathcal{P}_{0}(\Omega)\right]^{2 \times 2}
$$

and

$$
\oint_{E} \nabla_{E, k} \otimes \mathbf{v d} \mathbf{X}=\int_{E} \nabla \mathbf{v d} \mathbf{X}, \quad \forall \mathbf{v} \in\left[\mathcal{M}_{1}(E)\right]^{2}
$$


we have for any trial displacement field $\mathbf{v}_{h} \in \mathcal{K}_{h, k}^{0}$

$$
\begin{aligned}
G_{h}\left(\mathbf{p}_{1}, \mathbf{v}_{h}\right) & =\sum_{E \in \Omega} \oint_{E} \frac{\partial W}{\partial \mathbf{F}}\left(\mathbf{X}, \mathbf{I}+\nabla \mathbf{p}_{1}\right): \nabla_{E, k} \mathbf{v}_{h} \mathrm{~d} \mathbf{X}-\int_{\Gamma^{\mathbf{t}}} \mathbf{t}_{h} \cdot \mathbf{v}_{h} \mathrm{~d} S \\
& =\sum_{E \in \Omega} \int_{E} \frac{\partial W}{\partial \mathbf{F}}\left(\mathbf{X}, \mathbf{I}+\nabla \mathbf{p}_{1}\right): \nabla \mathbf{v}_{h} \mathrm{~d} \mathbf{X}-\int_{\Gamma^{\mathbf{t}}} \mathbf{t} \cdot \mathbf{v}_{h} \mathrm{~d} S \\
& =\int_{\Omega} \frac{\partial W}{\partial \mathbf{F}}\left(\mathbf{X}, \mathbf{I}+\nabla \mathbf{p}_{1}\right): \nabla \mathbf{v}_{h} \mathrm{~d} \mathbf{X}-\int_{\Gamma^{\mathbf{t}}} \mathbf{t} \cdot \mathbf{v}_{h} \mathrm{~d} S=0
\end{aligned}
$$

In the similar manner, we are able to show that for any $\mathbf{v}_{h} \in \mathcal{K}_{h, k}^{0}$,

$$
\begin{array}{r}
G_{h}^{\mathbf{u}}\left(\mathbf{p}_{1}, \widehat{p}_{0}, \mathbf{v}_{h}\right)=\sum_{E \in \Omega} \oint_{E}\left\{-\frac{\partial \widehat{W}^{*}}{\partial \mathbf{F}}\left(\mathbf{X}, \mathbf{I}+\nabla \mathbf{p}_{1}, \widehat{p}_{0}\right)+\widehat{p}_{0} \frac{\partial}{\partial \mathbf{F}}\left[\operatorname{det}\left(\mathbf{I}+\nabla \mathbf{p}_{1}\right)\right]\right\}: \nabla_{E, k} \mathbf{v}_{h} \mathrm{~d} \mathbf{X} \\
-\int_{\Gamma^{\mathrm{t}}} \mathbf{t}_{h} \cdot \mathbf{v}_{h} \mathrm{~d} S=0,
\end{array}
$$

and for any $\widehat{q}_{h} \in \mathcal{Q}_{h, k-1}^{D}\left(\right.$ or $\left.\mathcal{Q}_{h, k-1}^{C}\right)$,

$$
G_{h}^{\widehat{p}}\left(\mathbf{p}_{1}, \widehat{p}_{0}, \widehat{q}_{h}\right)=\sum_{E \in \Omega} \oint_{E}\left[\operatorname{det}\left(\mathbf{I}+\nabla \mathbf{p}_{1}\right)-1-\frac{\partial \widehat{W}^{*}}{\partial \widehat{p}}\left(\mathbf{X}, \mathbf{I}+\nabla \mathbf{p}_{1}, \widehat{p}_{0}\right)\right] \widehat{q}_{h} \mathrm{~d} \mathbf{X}=0
$$

We note the equality (54) comes from the the fact that

$$
\operatorname{det}\left(\mathbf{I}+\nabla \mathbf{p}_{1}\right)-1-\frac{\partial \widehat{W}^{*}}{\partial \widehat{p}}\left(\mathbf{X}, \mathbf{I}+\nabla \mathbf{p}_{1}, \widehat{p}_{0}\right)=0
$$

according to (49) and the definition of $\widehat{W}^{*}$. As a result, both displacement-based and mixed approximations of order $k(k \geq 1)$ exactly pass the first order patch test. In fact, our numerical studies in the subsequent section confirm that the first order patch test is passed up to machine precision errors.

In contrast, higher order patch test may not be exactly passed in general for finite elasticity problems because of the general forms that $\partial W / \partial \mathbf{F}, \partial \widehat{W}^{*} / \partial \mathbf{F}$ and $\partial \widehat{W}^{*} / \partial \widehat{p}$ may take. However, the following analysis demonstrates that the associated consistency errors converge to zero with the same rate as the finite element approximation errors (we recall that the approximation errors are typical order $k$ for the $k$ th order element) [55], implying the higher order patch test will be passed asymptotically with mesh refinement. For instance, in the $k$ the order patch test, the exact displacement is taken as a $k$ the order polynomial field, i.e., $\mathbf{u}=\mathbf{p}_{k} \in\left[\mathcal{P}_{k}(\Omega)\right]^{2}$, and the body force $\mathbf{f}$, boundary traction $\mathbf{t}$, and pressure field $\widehat{p}$ can be computed accordingly through constitutive and equilibrium equations. For displacement-based finite element approximation, 
we have for any $\mathbf{v}_{h} \in \mathcal{K}_{h, k}^{0}$,

$$
\begin{aligned}
G_{h}\left(\mathbf{p}_{k}, \mathbf{v}_{h}\right) & =\sum_{E \in \Omega} \oint_{E} \frac{\partial W}{\partial \mathbf{F}}\left(\mathbf{X}, \mathbf{I}+\nabla \mathbf{p}_{k}\right): \nabla_{E, k} \mathbf{v}_{h} \mathrm{~d} \mathbf{X}-\oint_{\Omega} \mathbf{f}_{h} \cdot \mathbf{v}_{h} \mathrm{~d} \mathbf{X}-\int_{\Gamma^{\mathbf{t}}} \mathbf{t}_{h} \cdot \mathbf{v}_{h} \mathrm{~d} S \\
& =\int_{\Omega} \frac{\partial W}{\partial \mathbf{F}}\left(\mathbf{X}, \mathbf{I}+\nabla \mathbf{p}_{k}\right): \nabla \mathbf{v}_{h} \mathrm{~d} \mathbf{X}+\mathcal{O}\left(h^{k}\right)\left\|\nabla \mathbf{v}_{h}\right\|-\oint_{\Omega} \mathbf{f}_{h} \cdot \mathbf{v}_{h} \mathrm{~d} \mathbf{X}-\int_{\Gamma^{\mathbf{t}}} \mathbf{t}_{h} \cdot \mathbf{v}_{h} \mathrm{~d} S \\
& =\left(\int_{\Omega} \mathbf{f} \cdot \mathbf{v}_{h} \mathrm{~d} \mathbf{X}-\oint_{\Omega} \mathbf{f}_{h} \cdot \mathbf{v}_{h} \mathrm{~d} \mathbf{X}\right)+\left[\int_{\Gamma^{\mathbf{t}}}\left(\mathbf{t}-\mathbf{t}_{h}\right) \cdot \mathbf{v}_{h} \mathrm{~d} S\right]+\mathcal{O}\left(h^{k}\right)\left\|\nabla \mathbf{v}_{h}\right\| \\
& =\mathcal{O}\left(h^{k}\right)\left\|\nabla \mathbf{v}_{h}\right\|,
\end{aligned}
$$

where the second equality comes from the estimate (39) and the second to last equality is a consequence of the assumed exactness requirements of the volume and boundary integration scheme $[56,57]$. For mixed approximation, we can also show in the similar manner that,

$$
G_{h}^{\mathbf{u}}\left(\mathbf{p}_{k}, \widehat{p}, \mathbf{v}_{h}\right)=\mathcal{O}\left(h^{k}\right)\left\|\nabla \mathbf{v}_{h}\right\|
$$

for any $\mathbf{v}_{h} \in \mathcal{K}_{h, k}$, and, based on the assumed exactness of volumetric integral $\oint_{E}$,

$$
G_{h}^{\widehat{p}}\left(\mathbf{p}_{k}, \widehat{p}, \widehat{q}_{h}\right)=\mathcal{O}\left(h^{k}\right)\left\|\widehat{q}_{h}\right\|
$$

for any $\widehat{q}_{h} \in \mathcal{Q}_{h, k-1}^{D}\left(\right.$ or $\left.\mathcal{Q}_{h, k-1}^{C}\right)$. Although not presented in this work, our numerical studies indicate that both displacement and mixed approximations indeed asymptotically pass the higher order patch test with their respective optimal convergence rates.

\section{Numerical Assessment}

In this section, we present a series of numerical tests to assess the performance of the displacement-based and two-field mixed polygonal elements. Both linear and quadratic elements are considered and investigated. Through the patch test and convergence studies, the effectiveness of the gradient correction scheme in ensuring the convergence of polygonal finite element solutions is demonstrated. Moreover, for mixed polygonal elements, numerical evaluations and discussions on the numerical stability and accuracy for different choice of pressure approximations are also provided.

Throughout the section, plane strain conditions are assumed and material behavior is considered to be Neo-Hookean as characterized by the following stored-energy function:

$$
W(\mathbf{F})=\frac{\mu}{2}[\mathbf{F}: \mathbf{F}-3]-\mu(\operatorname{det} \mathbf{F}-1)+\frac{3 \kappa+\mu}{6}(\operatorname{det} \mathbf{F}-1)^{2},
$$

where $\mu$ and $\kappa$ denote the initial shear and bulk moduli of the material response. The corre- 
sponding Legendre transformation (8) is given by

$$
\widehat{W}^{*}(\mathbf{F}, \widehat{q})=-\frac{\mu}{2}[\mathbf{F}: \mathbf{F}-3]+\frac{3(\mu+\widehat{q})^{2}}{2(3 \kappa+\mu)}
$$

Unless otherwise stated, triangulation rules with minimal required orders of accuracy are adopted, which we recall are 1st and 2nd order for linear and quadratic polygonal elements, respectively. In terms of the technique for solving the nonlinear system of equations, the standard Newton-Raphson method is employed and each loading step is regarded as converged once the norm of the residual reduces below $10^{-8}$ times that of the initial residual. The polygonal meshes used in this section are generated by the general purpose mesh generator for polygonal elements "PolyMesher" [58].

\subsection{Displacement-based polygonal finite elements}

In this subsection, we provide numerical experiments assessing the performance of the displacement-based polygonal elements with the gradient correction scheme. For comparison purposes, results from triangulation rules but without the correction of the gradients are also provided. In all of the examples considered we use $\mu=\kappa=1$. Two global error measures are adopted, the $\mathcal{L}^{2}$-norms and $\mathcal{H}^{1}$-seminorms of the displacement field errors, which are defined as

$$
\epsilon_{0, \mathbf{u}}=\left\|\mathbf{u}-\mathbf{u}_{h}\right\| \text { and } \epsilon_{1, \mathbf{u}}=\left\|\nabla \mathbf{u}-\nabla \mathbf{u}_{h}\right\|,
$$

and evaluated with an 8th order triangulation rule in all the remaining numerical examples.

Patch test. We begin with the standard patch test on a unit square domain $\Omega=(0,1)^{2}$, as depicted in Fig. 5 (a). On the boundary of the unit square $\partial \Omega$, an exact displacement field is applied, which is linear in both $X_{1}$ and $X_{2}$ directions:

$$
u_{1}(\mathbf{X})=2 X_{1}, \quad u_{2}(\mathbf{X})=-0.5 X_{2}
$$

Structured polygonal meshes and the centroid Voronoi Tessellation (CVT) meshes are considered, as shown in Fig. 5 (b) and (c). Each of the structured polygonal mesh consists of hexagons in the interior and pentagons and quadrilaterals on the boundary. In order to take into account irregular element shapes, the elements in the mesh are slightly elongated in $X_{1}$ direction.

We summarize the numerical results of the patch test in Tables 1 and 2. In the tables, only the more representative $\mathcal{H}^{1}$-seiminorm of the displacement error is presented, and each data for the CVT mesh is obtained by taking average of errors from a set of three meshes. Without applying the gradient correction scheme, the errors for both linear and quadratic polygonal 
elements stay constant over the mesh refinement, indicating that the patch test is not passed. Although not presented here, we have observed the same non-vanishing consistency errors with triangulation rules of higher order. In contrast, the errors remain close to machine precision levels for both linear and quadratic polygonal elements when the gradients are corrected, indicating that the patch test is passed. For quadratic elements, an evident accumulation of numerical errors under mesh refined, which is possibly due to the accumulation of numerical errors in computing the shape functions and their gradients. We note that the similar behavior has also been observed in references $[1,4]$, where the same construction of quadratic shape function is adopted as this paper.

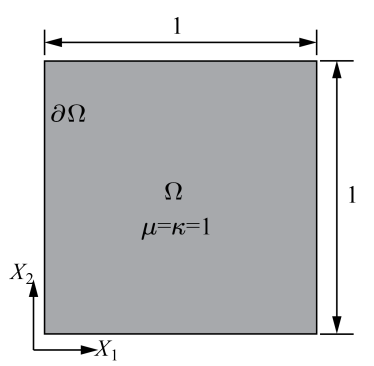

(a)

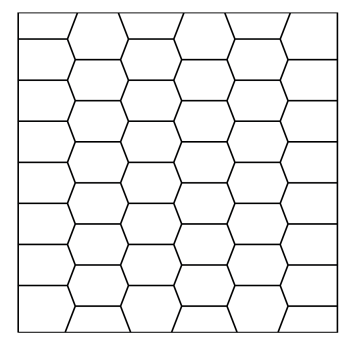

(b)

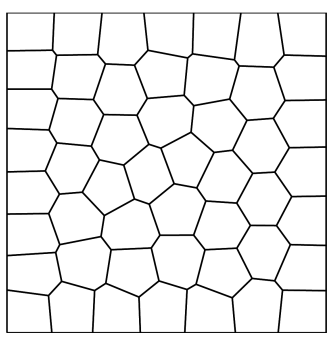

(c)

Figure 5: (a) Problem setting for the patch test. (b) An illustration of the structured hexagonal-dominant mesh with 48 elements. (c) An illustration of the CVT mesh with 50 elements.

Table 1: Results of $\epsilon_{1, \mathbf{u}}$ for the patch test for both linear and quadratic polygonal elements on structured hexagonal-dominant meshes

\begin{tabular}{cc|c|c|c|c}
\hline \hline \multirow{2}{*}{ \# element } & \multirow{2}{*}{$h$} & \multicolumn{2}{|c|}{ Linear polygonal elements } & \multicolumn{2}{c}{ Quadratic polygonal elements } \\
\cline { 3 - 6 } & & Uncorrected & Corrected & Uncorrected & Corrected \\
\hline 130 & 0.088 & 0.1168 & $5.16 E-14$ & $1.15 E-2$ & $4.77 E-13$ \\
520 & 0.044 & 0.1282 & $1.03 E-13$ & $1.08 E-2$ & $3.88 E-12$ \\
2080 & 0.022 & 0.1339 & $2.15 E-13$ & $1.03 E-2$ & $3.38 E-11$ \\
18720 & 0.0073 & 0.1377 & $5.99 E-13$ & $9.98 E-3$ & $9.10 E-10$ \\
\hline
\end{tabular}

Table 2: Results of $\epsilon_{1, \mathbf{u}}$ for the patch test for both linear and quadratic polygonal elements on CVT meshes

\begin{tabular}{cc|c|c|c|c}
\hline \hline \multirow{2}{*}{ \# element } & \multirow{2}{*}{$h$} & \multicolumn{2}{|c|}{ Linear polygonal elements } & \multicolumn{2}{c}{ Quadratic polygonal elements } \\
\cline { 3 - 6 } & & Uncorrected & Corrected & Uncorrected & Corrected \\
\hline 100 & 0.1 & 0.141 & $4.16 E-14$ & $2.27 E-2$ & $2.99 E-13$ \\
500 & 0.045 & 0.118 & $9.25 E-14$ & $1.18 E-2$ & $3.35 E-12$ \\
2000 & 0.022 & 0.119 & $1.86 E-13$ & $1.24 E-2$ & $2.64 E-11$ \\
20000 & 0.0071 & 0.119 & $5.92 E-13$ & $1.16 E-2$ & $8.56 E-10$ \\
\hline
\end{tabular}

Convergence study. A convergence study is performed in which we consider a boundary value problem where a rectangular block of size $\frac{\pi}{3} \times \pi$ is subjected to a certain distribution of body 
forces so as to be bent into a semicircle; see Fig. 6 (a). More precisely, the displacement field is given by

$$
u_{1}(\mathbf{X})=-1+\left(1+X_{1}\right) \cos \left(X_{2}\right)-X_{1}, \quad u_{2}(\mathbf{X})=\left(1+X_{1}\right) \sin \left(X_{2}\right)-X_{2},
$$

and the body force by

$$
f_{1}(\mathbf{X})=-\frac{\cos \left(X_{2}\right)\left(X_{1}+1\right)(3 \kappa-2 \mu)}{3}, \quad f_{2}(\mathbf{X})=-\frac{\sin \left(X_{2}\right)\left(X_{1}+1\right)(3 \kappa-2 \mu)}{3}
$$

Similar to the patch test discussed above, we first make use of hexagonal-dominated meshes, an example of which is displayed in Fig. 6 (b). In order to investigate the effect of the integration order on the convergence and accuracy of the results, we also consider the integration rules that are one order higher than the minimal required one, namely 2 nd order for linear elements and 3rd order for quadratic elements. The convergence results are summarized in Figs. 7 (a)-(d). For linear polygonal elements, it is clear from the figures that the 1st order integration is not a sufficient scheme to ensure optimal convergence of the finite element solutions without the gradient correction scheme. The $\mathcal{L}^{2}$-norm of the displacement error shows severely deteriorated convergence and a lack of convergence is observed for the $\mathcal{H}^{1}$-seminorm of the displacement error. This is due to the dominance of consistency errors observed in the patch test, which do not vanish under mesh refinement. The 2nd order triangulation rule, on the other hand, seems sufficient to ensure enough accuracy and optimal convergence for the range of mesh sizes considered even without the gradient correction scheme. We should note, however, that with further refinement of the mesh, the consistency error will gradually become dominant and the convergence rates of the error norms are expected to decrease accordingly. Unfortunately, it is not the case for quadratic polygonal elements. Without the correction of gradients, both 2nd and 3rd order triangulation rules show severe deteriorated convergence in the $\mathcal{L}^{2}$-norm of the displacement error and non-convergence in the $\mathcal{H}^{1}$-seminorm of the displacement error, which indicates that the consistency error plays a dominant role on the convergence of the finite element solutions for quadratic polygonal elements.

With the application of the gradient correction scheme, on the contrary, we recover the optimal convergence rates for both linear and quadratic polygonal elements, namely 2 and 1 for the $\mathcal{L}^{2}$-norm and $\mathcal{H}^{1}$-seminorm of the displacement errors, respectively, for linear elements, and 3 and 2 for those of the quadratic elements, respectively. Another key observation is that the gradient correction scheme allows the usage of the minimal required order of integration to achieve the same level of accuracy as with higher order integrations. As shown in Figs. 7 (a) and (b), the error norms are almost identical for 1st and 2nd order triangulations rules for linear polygonal elements when the gradients are corrected. This suggests that a 1st order 


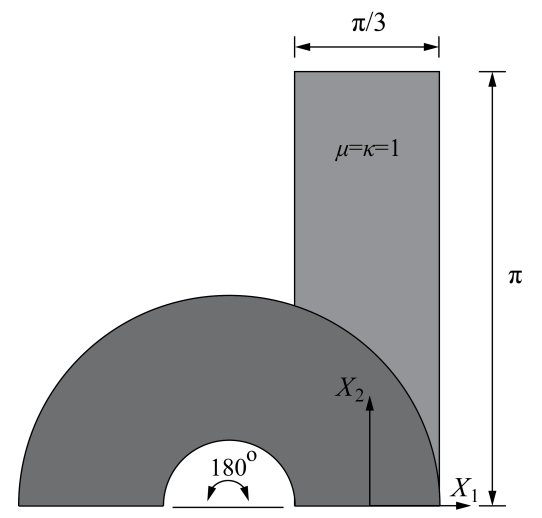

(a)

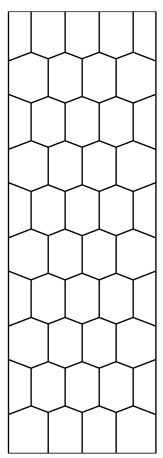

(b)

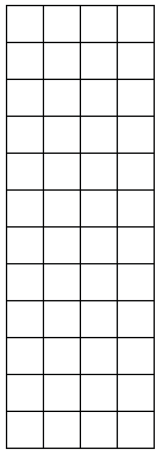

(f)

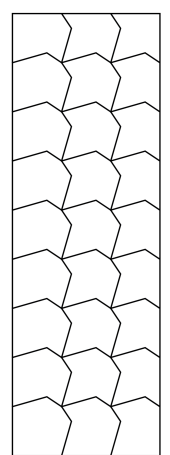

(c)

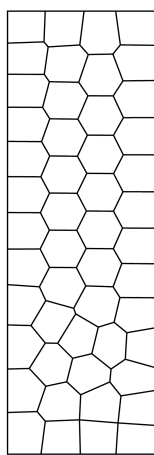

(d)

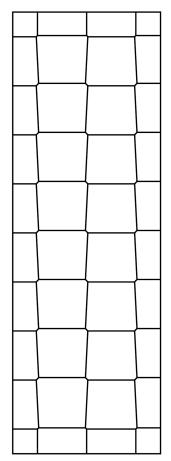

(e)

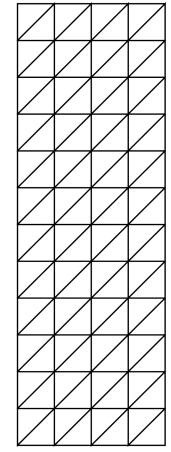

(g)

Figure 6: (a) Schematic of the bending of a compressible $(\mu=\kappa=1)$ rectangular block into semicircular shape. (b) An example of structured hexagonal-dominant mesh consisting of 45 elements. (c) An example of the concave octagonal mesh consisting of 27 elements. (d) An example of the CVT mesh consisting of 50 elements. (e) An example of the degenerated Voronoi mesh with small edges consisting 40 elements. (f) An example of the structured quadrilateral mesh consisting of 48 elements. (g) An example of the triangular mesh consisting of 96 elements.

integration rule with gradient correction can be used in practice without sacrificing accuracy, which leads to more efficient implementations. Typically, a triangulation rule of order 1 contains $n$ integration points for a $n$-gon. Compared to the commonly used 2nd order triangulation rule for linear polygons in the literature, which contains $3 n$ integration points per $n$-gon instead, the 1st order triangulation rule can roughly reduce two thirds of the computational cost in forming the stiffness matrices and internal force vectors. The same observations are also made for the quadratic polygonal elements, i.e, the solutions errors are almost identical for 2nd and 3rd integration rules. This indicates that, when the gradient correction scheme is applied, the minimal required 2nd order integration is also sufficient in practice for quadratic polygonal elements.

We also consider a set of concave meshes, an example of which is shown in Fig. 6 (c) and use the minimal required integration orders, which are 1st and 2nd orders for linear and quadratic polygonal elements respectively. The numerical results are shown in Fig. 8 (a) and 


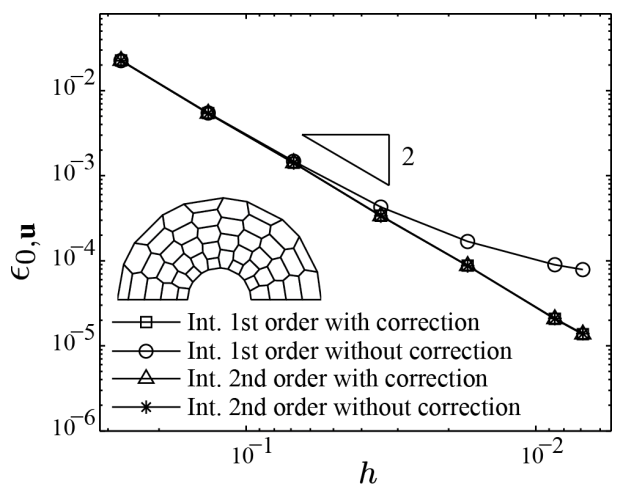

(a)

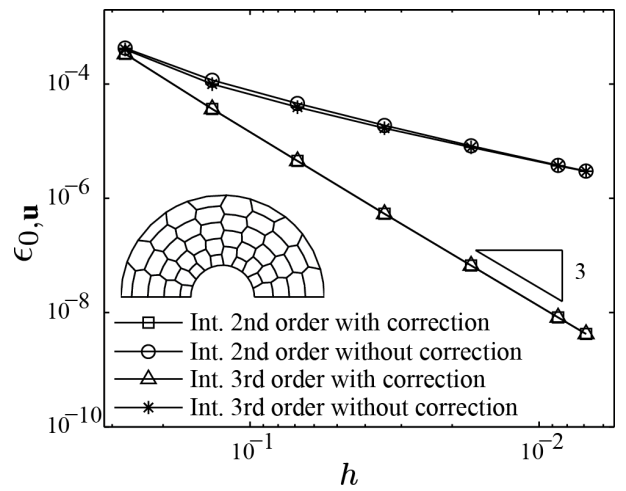

(c)

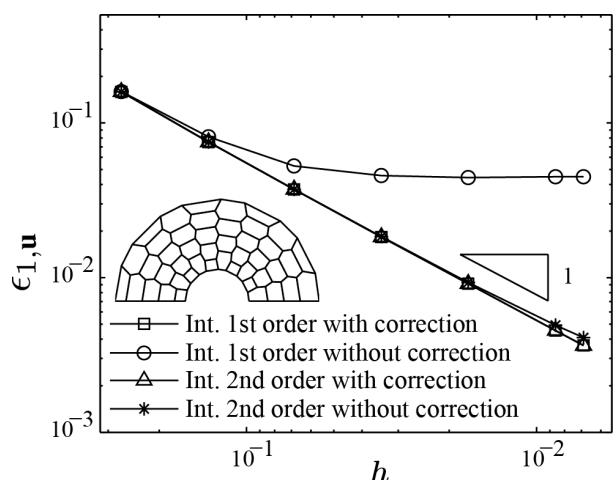

(b)

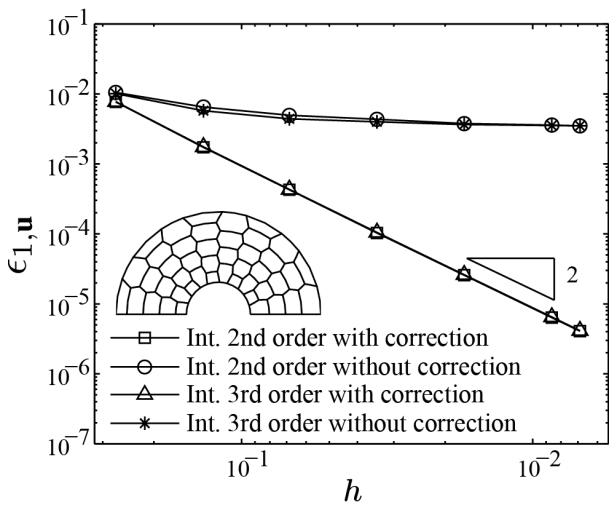

(d)

Figure 7: Plots of the error norms against the average mesh size $h$ for the structured hexagonal-dominant meshes: (a) the $\mathcal{L}^{2}$-norm of the error in the displacement field for linear polygonal elements, (b) the $\mathcal{H}^{1}$-seminorm of the error in the displacement field for linear polygonal elements, (c) the $\mathcal{L}^{2}$-norm of the error in the displacement field for quadratic polygonal elements and (d) The $\mathcal{H}^{1}$-seminorm of the error in the displacement field for quadratic polygonal elements.

(b), which confirm the optimal convergence rates when the gradients are corrected. In fact, the optimal convergence implies the applicability of the quadratic shape functions and the gradient correction scheme adopted in this work to certain concave polygonal elements.

We conclude this subsection with a brief study on the accuracy of the polygonal elements. The polygonal meshes adopted are the CVT meshes and degenerated Voronoi meshes with small edges, as shown in Figs. 6 (d) and (e) respectively. Both linear and quadratic polygonal elements are considered. For comparison purpose, we also include the quadrilateral and triangular meshes, examples of which are shown in Figs. 6 (f) and (g). Similarly, the triangular and quadrilateral finite elements use the standard iso-parametric construction and are up to quadratic order (for quadratic quadrilateral elements, we use the 8-node serendipity elements). For each type of element, we plot in Figs. 9 (a) and (b) the error norms $\epsilon_{0, \mathbf{u}}$ and $\epsilon_{1, \mathbf{u}}$ against the total number of nodes under the refinement of mesh, which reflects the size of the global system of equations and thus correlates with the cost of solving them. Each data point for the CVT 


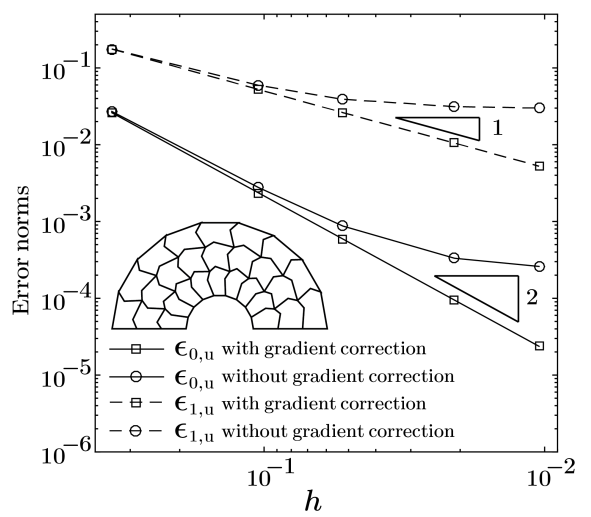

(a)

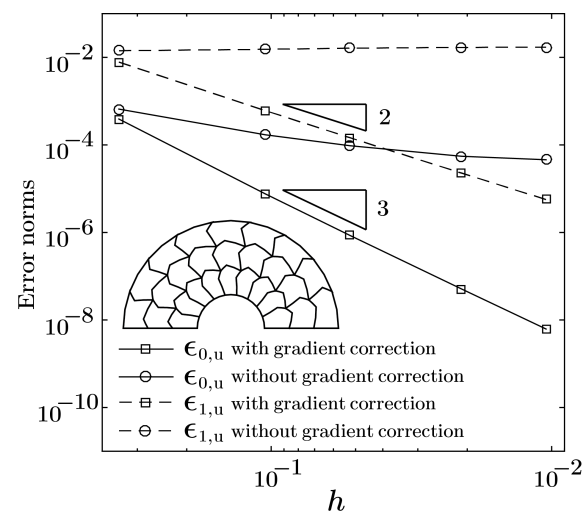

(b)

Figure 8: Plots of the error norms against the average mesh size $h$ for the concave octagonal meshes: (a) the $\mathcal{L}^{2}$-norm and $\mathcal{H}^{1}$-seminorm of the displacement errors for linear polygonal elements, and (b) the $\mathcal{L}^{2}$-norm and $\mathcal{H}^{1}$-seminorm of the displacement errors for quadratic polygonal elements. Only the minimal required orders of integration are used here, i.e., 1st order for the linear polygonal elements and 2nd order for the quadratic ones.

meshes is obtained from an average of the errors in three meshes. As we can see from the results in the Figs. 9 that the structured quadrilateral meshes provides the most accurate solutions for a given number of nodes in both linear and quadratic cases. This may be attributed to the fact that the exact displacement field for this problem is multiplicatively separable in $X_{1}$ and $X_{2}$ and is thus particularly well-suited for approximation by the tensor product in structured quadrilateral meshes. In terms of the $\mathcal{L}^{2}$-norm of the displacement error, the polygonal meshes (the CVT mesh and degenerated Voronoi meshes with small edges) yields similar accuracy to the triangular meshes in the linear case, where as in the quadratic case, they are more accurate than the triangular meshes. One the other hand, in terms of the $\mathcal{H}^{1}$-seminorm of the displacement error, the polygonal meshes yields more accurate results than the triangular meshes in both linear and quadratic cases, meaning that the polygonal meshes are able to approximate the gradient of the displacement field more accurately. Moreover, the comparison between the results from the CVT and degenerated Voronoi meshes with small edges shows that the effect of small edges in the accuracy of polygonal elements are small, indicating the polygonal meshes are tolerant to the presence of small edges.

\subsection{Two-field mixed polygonal element}

In this subsection, together with the gradient correction scheme, the performance of two field mixed polygonal elements on stability, accuracy and convergence are numerically evaluated. Three types of mixed polygonal finite elements are considered here, namely, $\mathcal{M}_{1}-\mathcal{P}_{0}^{D}$ elements, $\mathcal{M}_{2}-\mathcal{P}_{1}^{D}$ elements, and $\mathcal{M}_{2}-\mathcal{M}_{1}$ elements. In this subsection, the material is considered to be incompressible with $\mu=1$ and $\kappa=\infty$ and thus characterized by the standard Neo-Hookean 


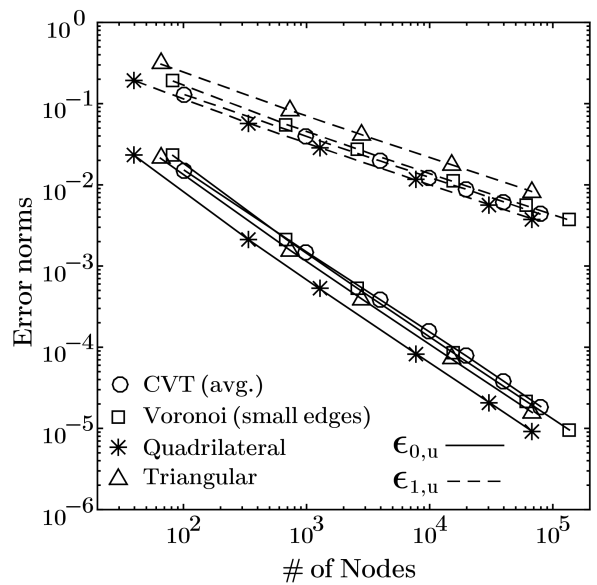

(a)

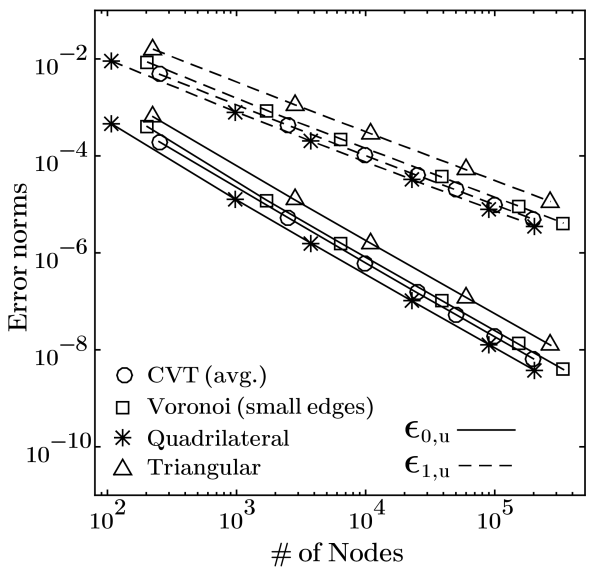

(b)

Figure 9: Comparisons of the error norms against the average mesh size $h$ between polygonal meshes (CVT meshes and Voronoi meshes with small edges) and meshes with standard triangular and quadrilateral finite elements: (a) the $\mathcal{L}^{2}$-norm and $\mathcal{H}^{1}$-seminorm of the displacement errors for linear elements, and (b) the $\mathcal{L}^{2}$-norm and $\mathcal{H}^{1}$-seminorm of the displacement errors for polygonal elements. For the polygonal meshes the results are obtained width corrected gradients.

stored-energy function

$$
W(\mathbf{X}, \mathbf{F})=\left\{\begin{array}{ll}
\frac{\mu}{2}[\mathbf{F}: \mathbf{F}-3] & \text { if } \operatorname{det} \mathbf{F}=1 \\
+\infty & \text { otherwise }
\end{array} .\right.
$$

In addition to the measure of displacement errors defined in (61), we also consider the $\mathcal{L}^{2}$-norm of the errors in the pressure field,

$$
\epsilon_{0, \widehat{p}}=\left\|\widehat{p}-\widehat{p}_{h}\right\|
$$

Numerical stability for linear elasticity. For mixed finite element methods involving approximations of the displacement and pressure fields, the satisfaction of the stability condition is crucial to guarantee convergence $[11,13,12]$. In the context of finite elasticity, the stability condition is formally defined by the generalized inf-sup condition [13, 12]. It states that for a given $\mathbf{u}_{h} \in \mathcal{K}_{h}$, there exist a strictly positive, size independent constant $C_{0}$, such that

$$
\beta_{h}\left(\mathbf{u}_{h}\right)=\inf _{q_{h} \in \mathcal{Q}_{h, k-1}^{D \text { or } C}} \sup _{\mathbf{v}_{h} \in \mathcal{K}_{h, k}} \frac{\int_{\Omega} q_{h} \operatorname{adj}\left(\mathbf{I}+\nabla \mathbf{u}_{h}\right): \nabla \mathbf{v}_{h} \mathrm{~d} \mathbf{X}}{\left\|\nabla \mathbf{v}_{h}\right\|\left\|q_{h}\right\|} \geq C_{0}
$$

Note that the above condition depends on the deformation state $\mathbf{u}_{h}$ in addition to variations $\mathbf{v}_{h}$ and $\widehat{q}_{h}$, which is nontrivial to verify. Instead, we only verify the inf-sup condition for linear elasticity in this work, that is

$$
\beta_{h}^{0}=\inf _{q_{h} \in \mathcal{Q}_{h, k-1}^{D} \text { or } C} \sup _{\mathbf{v}_{h} \in \mathcal{K}_{h, k}} \frac{\int_{\Omega} q_{h} \operatorname{div} \mathbf{v}_{h} \mathrm{~d} \mathbf{X}}{\left\|\nabla \mathbf{v}_{h}\right\|\left\|q_{h}\right\|} \geq C_{0},
$$


which can be viewed as a special case of (67) when $\mathbf{u}_{h}=\mathbf{0}$. For meshes consisting of lower order polygonal mixed elements, Beirao da Veiga et al. [14] have derived a geometrical condition to guarantee the satisfaction of (68) if every internal node/vertex in the mesh is connected to at most three edges. For higher order mixed elements, however, the analogous condition is still an open question and is subjected to future research. Here, we numerically evaluate the stability of linear and quadratic mixed polygonal elements. To that end, we adopt the so called inf-sup test proposed by Chapelle and Bathe [16]. We note that while passing the inf-sup test only constitutes a necessary condition for the satisfaction of the inf-sup condition (68), its predictions are shown to reliably match the analytical results $[16,58]$. In the test, we consider a unit square domain with imposed boundary conditions as shown in Fig. 10 (a). Three families of Voronoitype meshes are considered here, namely the structured hexagonal, CVT and random Voronoi Meshes, as shown in Fig. 10 (b)-(d). In general, the Voronoi-type meshes satisfy the geometrical condition of Beirao da Veiga et al. [14], including the random Voronoi mesh considered here (although they may contain very small edges). However, we note that in several cases, Voronoi meshes from degenerated seeds alignments may be in violation of the geometrical condition and lead to the failure of the inf-sup condition, for instance, the case where the Voronoi seeds are aligned in a Cartesian grid, forming a Cartesian mesh. For all types of meshes considered, we compute the stability index $\beta_{h}^{0}$ of linear and both types of mixed polygonal elements and plot them as functions of the average mesh size $h$ in Figs. 10 (e)-(g). Each point in the plot for CVT and random Voronoi meshes represents an average of the results from a set of three meshes. As suggested by the results of the test, all three types of mixed polygonal finite elements are numerically stable on all families of meshes considered. For comparison purposes, the test results for most of the classical triangular and quadrilateral elements can be found in $[16,15]$ and hence are not listed here for the sake of conciseness. We note that while the classical linear and quadratic mixed elements with continuous pressure approximations (the T-H family) are unconditionally stable [11], most of those with discontinuous pressure approximations (the C-R family), such as lower order mixed triangular and quadrilateral elements, are numerically unstable $[16,15,11]$.

Patch test. A patch test study is performed on an unit square domain $\Omega=(0,1)^{2}$, which is subjected to an uniaxial displacement loading on its right edge, as shown in Fig. 11 (a). The analytical displacement is a linear field of the form:

$$
u_{1}(\mathbf{X})=2 X_{1}, \quad u_{2}(\mathbf{X})=-\frac{2}{3} X_{2}
$$

and the pressure type field $\widehat{p}$ defined in the $\mathbf{F}$-Formulation is a constant over the domain with a value of $\widehat{p}=-\frac{1}{9}$.

The same sets of structured polygonal meshes and CVT meshes are used as that in the 


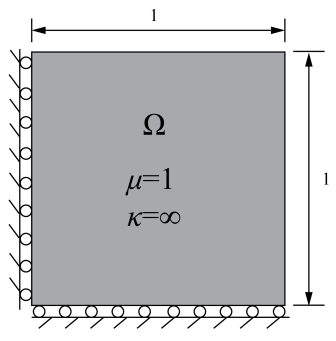

(a)

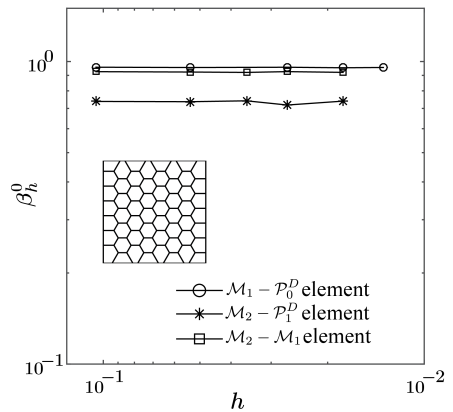

(e)

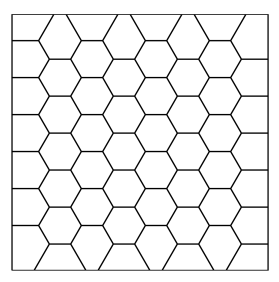

(b)

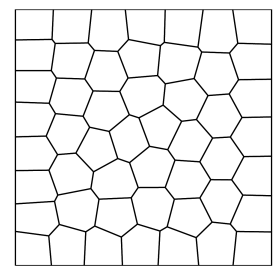

(c)

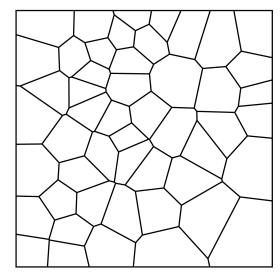

(d)

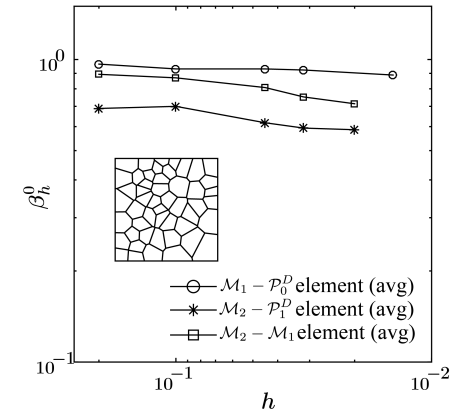

(g)

Figure 10: (a) Dimensions and boundary conditions for the Inf-Sup test. (b) An example of the structured hexagonal dominant mesh with 56 elements. (c) An example of the randomly generated CVT mesh with 50 elements. (d) An example of the random Voronoi mesh with 50 elements. (e) Plot of the computed value of the stability index as a function of the average mesh size $h$ for structured hexagonal dominant meshes. (f) Plot of the computed value of the stability index as a function of the average mesh size $h$ for CVT meshes. (g) Plot of the computed value of the stability index as a function of the average mesh size $h$ for random Voronoi meshes.

patch test study of the displacement-based elements, samples of which are shown in Fig. 11 (b) and (c). The patch test results are provided in Table 3-6 for linear and quadratic mixed polygonal elements. Again, with the gradient correction scheme, all the error norms stay close to machine precisions for all three type of mixed elements on both sets of meshes, which are not the case for those with uncorrected gradients.

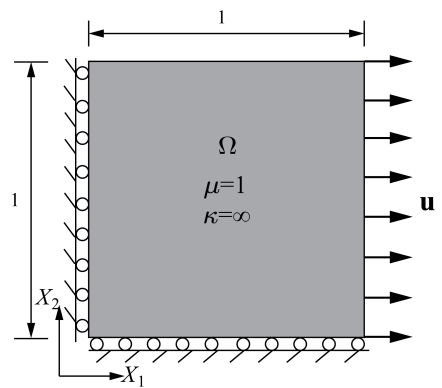

(a)

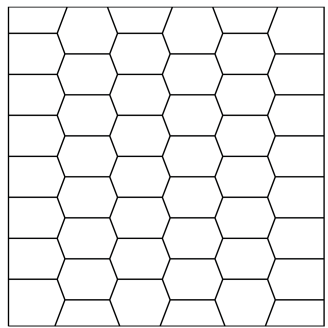

(b)

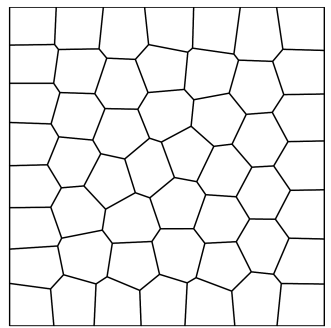

(c)

Figure 11: (a) Problem setting for first order patch test; (b) an example of the structured hexagonal-dominated mesh used in the patch test with 48 elements. (c) an example of the CVT mesh used in the patch test with 50 elements. 
Table 3: Results of patch test with gradient correction for mixed linear polygonal elements with structured hexagonal-dominant meshes

\begin{tabular}{cc|cc|cc}
\hline \hline$\#$ element & $h$ & \multicolumn{2}{|c|}{$\epsilon_{1, \mathbf{u}}$} & \multicolumn{2}{c}{$\epsilon_{0, \widehat{p}}$} \\
\hline $\mathcal{M}_{1}-\mathcal{P}_{0}^{D}$ & elements & Uncorrected & Corrected & Uncorrected & Corrected \\
\hline 130 & 0.088 & $1.87 E-01$ & $5.17 E-14$ & $1.08 E-02$ & $3.22 E-15$ \\
520 & 0.044 & $1.80 E-01$ & $1.05 E-13$ & $1.09 E-02$ & $7.07 E-15$ \\
2080 & 0.022 & $1.74 E-01$ & $2.20 E-13$ & $8.36 E-03$ & $1.34 E-14$ \\
18720 & 0.0073 & $1.70 E-01$ & $6.16 E-13$ & $4.55 E-03$ & $4.56 E-14$ \\
\hline
\end{tabular}

Table 4: Results of patch test with gradient correction for mixed linear polygonal elements with CVT meshes

\begin{tabular}{cc|cc|cc}
\hline \hline \# element & $h$ & \multicolumn{2}{|c|}{$\epsilon_{1, \mathbf{u}}$} & \multicolumn{2}{c}{$\epsilon_{0, \widehat{p}}$} \\
\hline $\mathcal{M}_{1}-\mathcal{P}_{0}^{D}$ & elements & Uncorrected & Corrected & Uncorrected & Corrected \\
\hline 100 & 0.088 & $1.56 E-01$ & $4.16 E-14$ & $4.95 E-03$ & $2.16 E-15$ \\
500 & 0.044 & $1.41 E-01$ & $9.71 E-14$ & $5.65 E-03$ & $5.46 E-15$ \\
2000 & 0.022 & $1.36 E-01$ & $1.91 E-13$ & $6.33 E-03$ & $1.30 E-14$ \\
20000 & 0.0073 & $1.46 E-01$ & $6.09 E-13$ & $6.47 E-03$ & $4.31 E-14$ \\
\hline
\end{tabular}

Table 5: Results of patch test with gradient correction for mixed quadratic polygonal elements with structured hexagonal-dominant meshes

\begin{tabular}{|c|c|c|c|c|c|}
\hline \# elemes & $h$ & \multicolumn{2}{|c|}{$\epsilon_{1, \mathbf{u}}$} & \multicolumn{2}{|c|}{$\epsilon_{0, \widehat{p}}$} \\
\hline $\mathcal{M}_{2}-\mathcal{P}_{1}^{\prime}$ & ments & Uncorrected & Corrected & Uncorrected & Corrected \\
\hline 130 & 0.088 & $1.37 E-02$ & 5.59 & -03 & 1.10 \\
\hline 520 & 44 & 2 & 3. & 4 & 13 \\
\hline 2080 & 022 & 02 & 3. & 4 & 12 \\
\hline 18720 & 0.0073 & $1.17 E-02$ & $9.26 E-10$ & 3.75 & $4.05 E-11$ \\
\hline $\mathcal{M}_{2}-\mathcal{N}$ & lements & Uncorrected & Corrected & Uncorrected & Corrected \\
\hline 130 & 0.088 & $1.37 E-02$ & $4.89 E-13$ & $9.68 E-04$ & $5.111 E-14$ \\
\hline 520 & 0.044 & $1.26 E-02$ & $3.93 E-12$ & $8.85 E-04$ & $2.02 E-13$ \\
\hline 2080 & 0.022 & $1.20 E-02$ & $3.43 E-11$ & $6.97 E-04$ & $1.00 E-12$ \\
\hline 18720 & 0.0073 & $1.17 E-02$ & $9.28 E-10$ & $4.27 E-04$ & $1.87 E-11$ \\
\hline
\end{tabular}

Convergence study. We proceed to evaluate the accuracy and convergence of the mixed polygonal finite elements with a boundary value problem where an incompressible rectangular block of dimensions $\frac{\pi}{3} \times \pi$ is bent into semicircular shape. For this boundary value problem, it is possible to work out an analytical solution [24]. Specifically, the displacement field and its gradient read as

$$
u_{1}=r\left(X_{1}\right) \cos \left(X_{2}\right)-r\left(-\frac{\pi}{6}\right)-\frac{\pi}{6}-X_{1}, \quad u_{2}=r\left(X_{1}\right) \sin \left(X_{2}\right)-X_{2}
$$


Table 6: Results of patch test with gradient correction for mixed quadratic polygonal elements with CVT meshes

\begin{tabular}{|c|c|c|c|c|c|}
\hline \# element & $h$ & \multicolumn{2}{|c|}{$\overline{\epsilon_{1, \mathbf{u}}}$} & \multicolumn{2}{|c|}{$\epsilon_{0, \widehat{p}}$} \\
\hline $\mathcal{M}_{2}-\mathcal{P}_{1}^{D}$ & ements & Uncorrected & Corrected & Uncorrected & Corrected \\
\hline 100 & 0.088 & $1.97 E-02$ & $3.75 E-13$ & $2.26 E-03$ & $1.08 E-13$ \\
\hline 500 & 0.044 & $1.65 E-02$ & $3.15 E$ & $1.68 E-03$ & $4.88 E-13$ \\
\hline 2000 & 0.022 & $1.43 E$ & 2.841 & & $3.34 E$ \\
\hline 20000 & 0.0073 & $1.40 E-02$ & $8.72 E-10$ & $1.37 E-03$ & $9.90 E-11$ \\
\hline $\mathcal{M}_{2}-\mathcal{M}_{1}$ & ements & Uncorrected & Corrected & Uncorrected & Corrected \\
\hline 100 & 0.088 & $1.56 E-02$ & $3.14 E-13$ & $1.90 E-04$ & $4.08 E-14$ \\
\hline 500 & 0.044 & $1.48 E-02$ & $3.40 E-12$ & $7.38 E-05$ & $1.68 E-13$ \\
\hline 2000 & 022 & $1.47 E-02$ & $2.68 E-11$ & $3.60 E-05$ & $8.15 E-13$ \\
\hline 20000 & 0.0073 & $1.44 E-02$ & $8.74 E-10$ & $1.14 E-05$ & $1.94 E-11$ \\
\hline
\end{tabular}

$$
\begin{aligned}
& u_{1,1}=\frac{\cos \left(X_{2}\right)}{r\left(X_{1}\right)}-1, \quad u_{1,2}=-r\left(X_{1}\right) \sin \left(X_{2}\right), \\
& u_{2,1}=\frac{\sin \left(X_{2}\right)}{r\left(X_{1}\right)}, \quad u_{2,2}=r\left(X_{1}\right) \cos \left(X_{2}\right)-1,
\end{aligned}
$$

where the function $r\left(X_{1}\right)$ is given by

$$
r\left(X_{1}\right)=\sqrt{2 X_{1}+\beta} \quad \text { with } \quad \beta=\sqrt{\frac{4 \pi^{2}}{9}+4}
$$

Additionally, one can also obtained the pressure field $\widehat{p}$ :

$$
\widehat{p}=-\frac{\mu}{2}\left[\frac{1}{r\left(X_{1}\right)^{2}}-r\left(X_{1}\right)^{2}+\mu \beta\right]
$$

Figure 12 (a) shows a schematic of the problem. In order to avoid the development of surface instabilities [59] and guarantee the uniqueness of the finite element solutions, the displacement is prescribed on the left side of the block.

In addition to the optimal convergence rate for the error measures of the displacement field discussed in previous subsections with displacement-based elements, we expect the optimal convergence rate for its $\mathcal{L}^{2}$ pressure error to be 1 for linear mixed elements and 2 for quadratic mixed elements with either $\mathcal{M}_{2}-\mathcal{P}_{1}^{D}$ or $\mathcal{M}_{2}-\mathcal{M}_{1}$ elements. The results of the convergence test are shown in Figs. 13 (a)-(i) for all three types of mixed finite elements considered. When no gradient correction scheme is used, we observe the same behavior regarding the convergence of displacement field solution as those of the displacement-based elements. In terms of the pressure field, although the 1st order integration leads to a degenerated convergence rate, the optimal convergence rate is obtained with the 2 nd order integration scheme for linear mixed elements. 


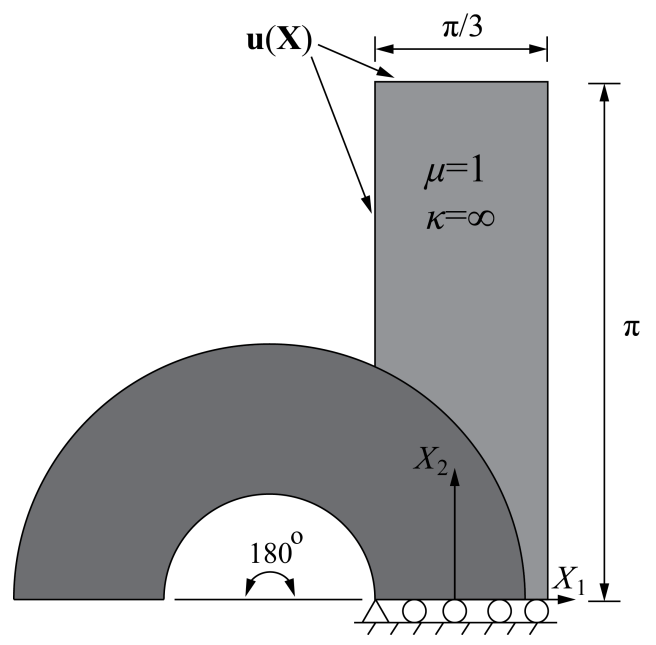

(a)

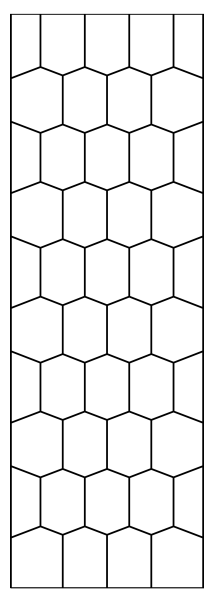

(b)

Figure 12: (a) Schematic of the bending of an incompressible $(\mu=1$ and $\kappa=\infty)$ rectangular block into semicircular shape. (b) An example mesh utilized in the convergence tests with 45 elements [58].

For quadratic mixed elements, however, the degeneration of the convergence rate becomes more severe and increasing the integration order again proves to be not as helpful as in the linear case. In contrast, with the correction scheme, optimal convergence in both displacement field and pressure field are recovered for both linear and quadratic mixed elements. Again, the same level of accuracy for both displacement and pressure field is achieved with a minimal required order of triangulation rule.

We conclude this section by examining the accuracy of different types of mixed elements in enforcing the incompressibility constraint for the displacement field. To quantify the accuracy, we define $\epsilon_{0, J}$ as the $\mathcal{L}^{2}$-norm of the error between $\operatorname{det} \mathbf{F}$ and 1 ,

$$
\epsilon_{0, J}=\left\|\operatorname{det} \mathbf{F}\left(\mathbf{u}_{h}\right)-1\right\|
$$

Figures 14 (a) and (b) show the convergence of $\epsilon_{0, J}$ as a function of mesh size and total number of DOFs, respectively, for both hexagonal and CVT meshes [58]. For the CVT meshes, each data point is obtained by averaging values from a set of three meshes with the same number of elements. As we can see, the rate of convergence of $\epsilon_{0, J}$ to 0 is 1 for mixed linear polygonal elements and roughly 2 for both types of mixed quadratic polygonal elements. In addition, with either similar mesh size or total number of DOFs, $\mathcal{M}_{2}-\mathcal{M}_{1}$ elements seem to better enforce the incompressibility constraint on both hexagonal and CVT meshes for this problem.

\section{Applications}

This section presents physically-based applications of the above-developed polygonal finite elements. Two problems are studied: $(i)$ the nonlinear elastic response of an incompressible 


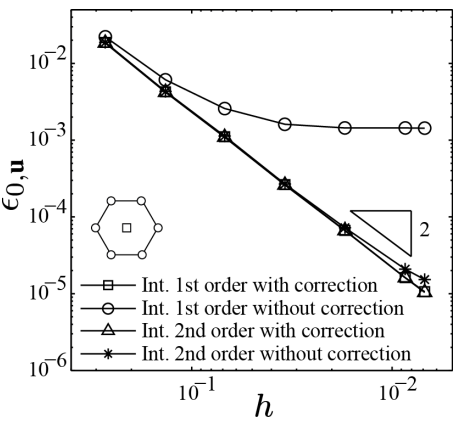

(a)

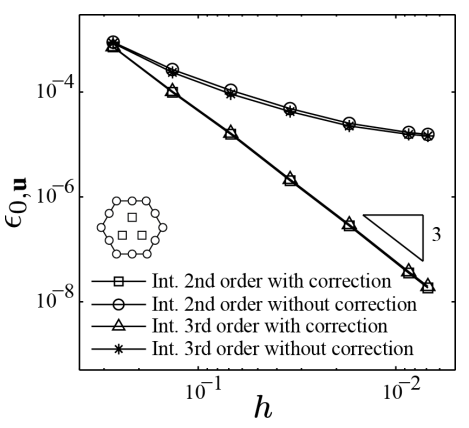

(d)

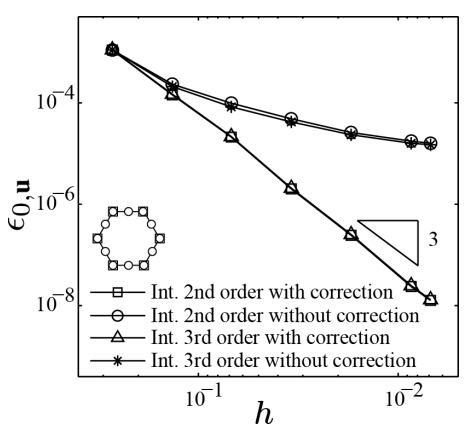

(g)

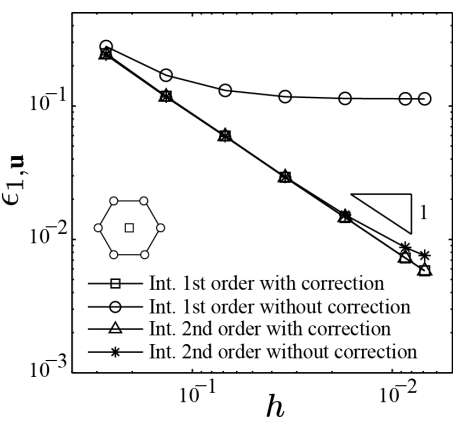

(b)

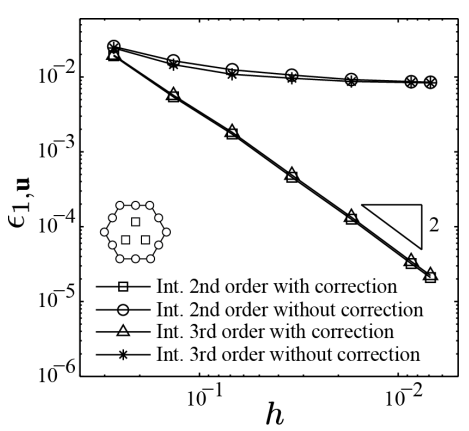

(e)

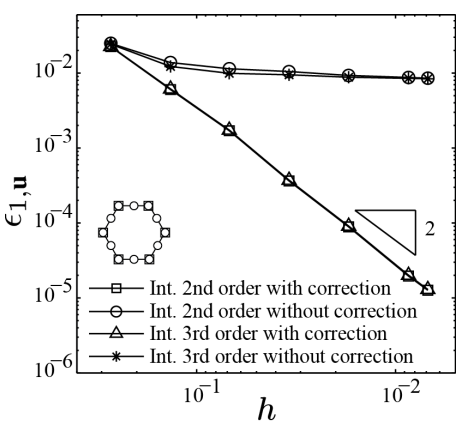

(h)

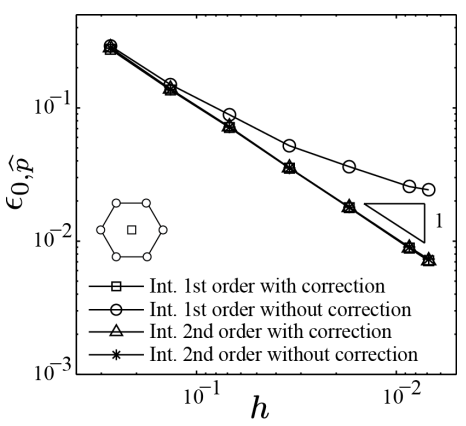

(c)

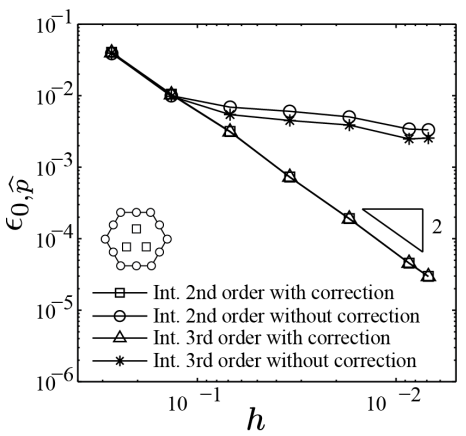

(f)

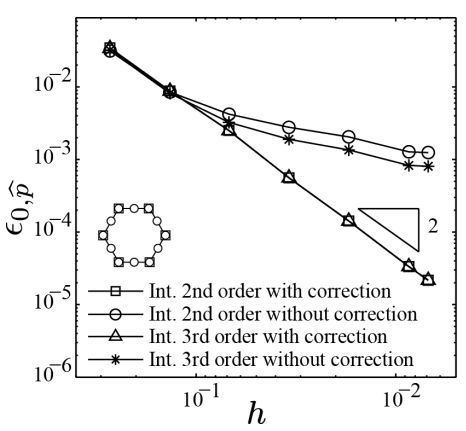

(i)

Figure 13: Plots of the error norms against the average mesh size $h$ : (a) the $\mathcal{L}^{2}$-norm of the displacement error, (b) the $\mathcal{H}^{1}$-seminorm of the displacement error, and (c) the $\mathcal{L}^{2}$-norm of pressure error for $\mathcal{M}_{1}-\mathcal{P}_{0}^{D}$ elements; (d) the $\mathcal{L}^{2}$-norm of the displacement error, (e) the $\mathcal{H}^{1}$-seminorm of the displacement error, and (f) the $\mathcal{L}^{2}$-norm of the pressure error for $\mathcal{M}_{2}-\mathcal{P}_{1}^{D}$ elements; (g) the $\mathcal{L}^{2}$-norm of the displacement error, (h) the $\mathcal{H}^{1}$-seminorm of the displacement error, and (i) the $\mathcal{L}^{2}$-norm of the pressure error for $\mathcal{M}_{2}-\mathcal{M}_{1}$ elements.

elastomer reinforced with a random isotropic distribution of circular rigid particles bonded through finite-size interphases, and $(i i)$ the onset of cavitation in a fiber reinforced elastomer. These example problems are aimed at demonstrating the ability of polygonal elements to model the complex behavior of nonlinear elastic materials over a wide range of length scales and deformations. For both examples, the inclusions are considered to be infinitely rigid and the variational formulation proposed by Chi et al. [60] is adopted, which treats the presence of 


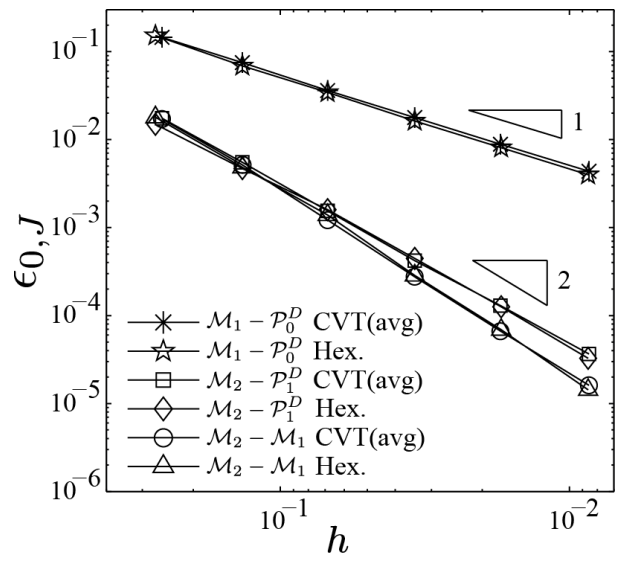

(a)

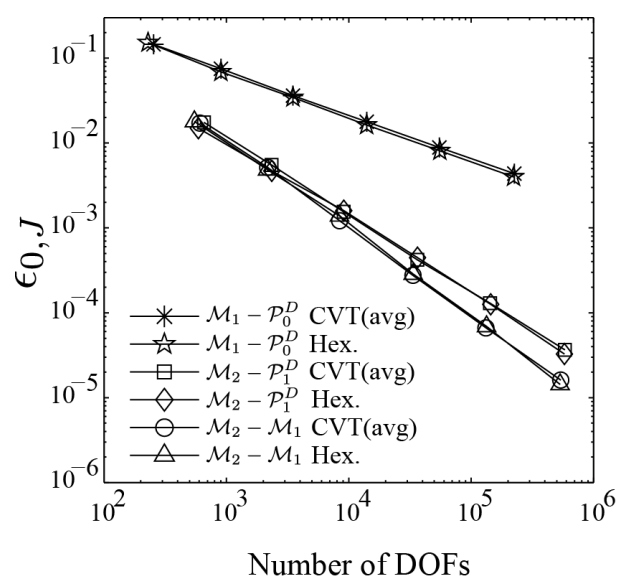

(b)

Figure 14: Plots of $\mathcal{L}^{2}$ error in satisfying the incompressibility constraint versus (a) the average mesh size $h$ and (b) the total number of DOFs.

rigid inclusions as a set of kinematic constraints on the displacement DOFs of their boundaries. Again, the polygonal meshes considered in this section are generated by "PolyMesher" [58].

\subsection{Elastomers reinforced with particles bonded through interphases}

Experimental evidence has by now established that filled elastomers contain stiff "interphases" or layers of stiff "bound rubber" around its inclusions [61], and that these can significantly affect their macroscopic response when the fillers are submicron in size [62, 63]. In most cases of practical interest, filler particles happen to be indeed submicron in size. In the sequel, motivated by the recent work of Goudarzi et al. [63], we investigate the nonlinear elastic response of an elastomer filled with a random isotropic distribution of circular particles bonded through finite-size stiff interphases by means of the polygonal finite element framework presented in this paper.

Figure 15 shows the unit cell that we consider for the problem at hand, which is assumed to be repeated periodically in the $\mathbf{e}_{1}$ and $\mathbf{e}_{2}$ directions so as to approximate a truly random and isotropic distribution of particles. The unit cell contains a total of 50 monodisperse rigid particles at an area fraction of $c_{p}=25 \%$. Each particle is bonded to the matrix through an interphase of constant thickness $t$, which is taken to be $20 \%$ of the particle radius, resulting in a total area fraction $c_{i}=11 \%$ of interphases. The matrix phase is modeled as an incompressible Neo-Hookean solid with stored-energy function given by (65) with $\mu_{m}=1$. Further, the constitutive behavior of the interphases is also taken as incompressible Neo-Hookean, but ten times stiffer than the matrix phase, namely, $\mu_{i}=10 \mu_{m}=10$.

We employ the commonly used mixed formulation in this example (the $\overline{\mathbf{F}}$-formulation) and study the nonlinear elastic behavior of such a material by polygonal meshes with linear and quadratic mixed elements [58]; see Figs. 15 (b) and (c). For the quadratic polygonal mesh, ele- 


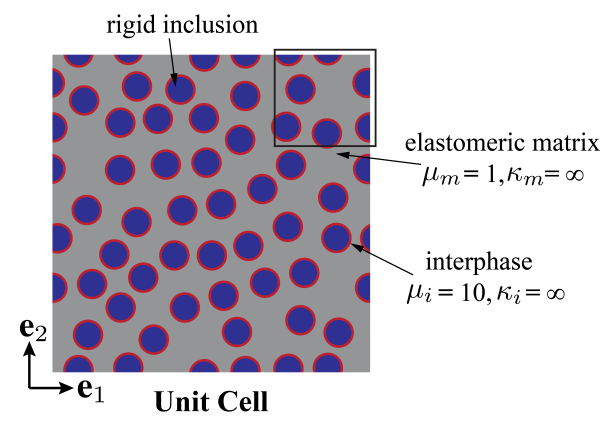

(a)

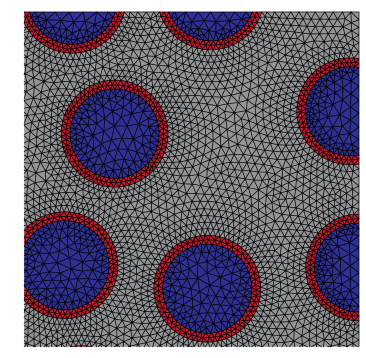

(d)

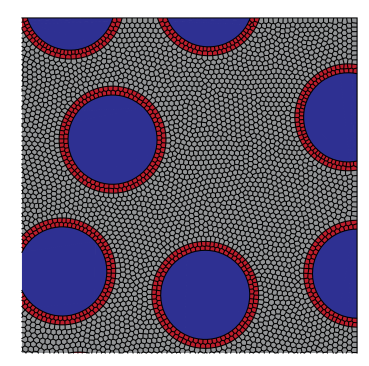

(b)

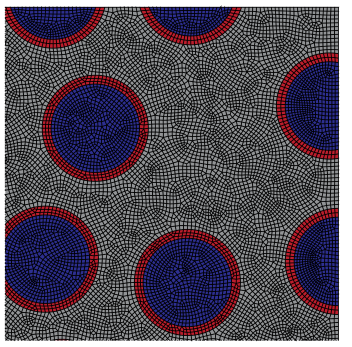

(e)

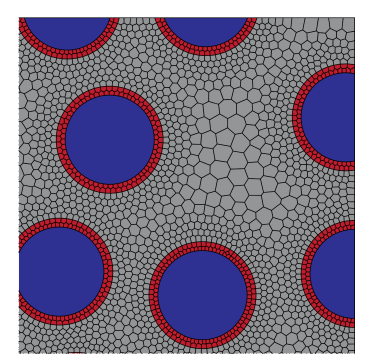

(c)

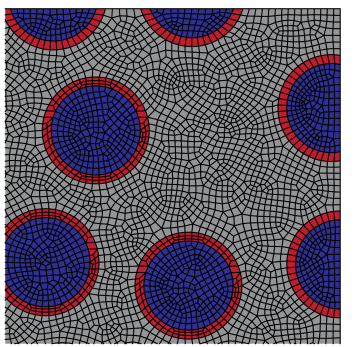

(f)

Figure 15: (a) The unit cell considered in the problem. (b) The linear polygonal mesh comprised of 39738 elements and 76020 nodes in total, with 66095 nodes in the matrix phase. (c) The quadratic polygonal mesh comprised of 20205 elements and 95233 nodes in total, with 67325 nodes in the matrix phase. (d) The quadratic triangular mesh comprised of 58814 CPE6MH elements and 118141 nodes in total, with 71179 nodes in the matrix phase. (e) The linear quadrilateral mesh comprised of $108742 \mathrm{CPE} 4 \mathrm{H}$ elements and 109323 nodes in total, with 68819 nodes in the matrix phase. (f) The quadratic quadrilateral mesh comprised of $36444 \mathrm{CPE} 8 \mathrm{H}$ elements and 109986 nodes in total, with 66502 nodes in the matrix phase.

ments with both discontinuous and continuous pressure approximations are considered, namely, the $\mathcal{M}_{2}-\mathcal{M}_{1}$ and $\mathcal{M}_{2}-\mathcal{P}_{1}^{D}$ elements. For comparison purposes, finite element meshes of 4-node hybrid linear quadrilateral elements (termed CPE4H), 6-node hybrid quadratic triangular elements (termed CPE6MH) and 8-node hybrid quadratic quadrilateral elements (termed $\mathrm{CPE} 8 \mathrm{H}$ ), are also included, the depictions of which can be found in Fig. 15 (d)-(f). For fair comparison, all the meshes are chosen such that they have a similar number of nodes in the matrix phase. For the polygonal mesh, we use the same convergence criterion as the other examples, that is, each loading step is regarded as converged once the norm of the residual reduces below $10^{-8}$ times that of the initial residual. Whereas for the results by ABAQUS, we use its default set of convergence criteria, among which the permissible ratio of the largest solution correction to the largest corresponding incremental solution is $|\Delta \mathbf{u}| /\left|\mathbf{u}_{\max }\right|=10^{-2}$, the largest residual to the corresponding average force norm is $5 \times 10^{-3}$. In addition, the largest permissible absolute error in satisfying the incompressiblity constraint is $10^{-5}$ in the ABAQUS default convergence criteria. 
The boundary conditions are as follows [62]

$$
\begin{aligned}
& u_{k}\left(1, X_{2}\right)-u_{k}\left(0, X_{2}\right)=\langle\mathbf{F}\rangle_{k 1}-\delta_{k 1} \\
& u_{k}\left(X_{1}, 1\right)-u_{k}\left(X_{1}, 0\right)=\langle\mathbf{F}\rangle_{k 2}-\delta_{k 2} \quad \forall k=1,2
\end{aligned}
$$

and

$$
p\left(1, X_{2}\right)=p\left(0, X_{2}\right), \text { and } p\left(X_{1}, 1\right)=p\left(X_{1}, 0\right) .
$$

In the above relations, $\delta_{k l}$ is the Kronecker delta, and $u_{k}$ and $X_{k}(k=1,2)$ stand for the components of the displacement field and initial position vector in a Cartesian frame of reference with its origin placed at the left lower corner of the unit cell. We consider two loading conditions: (i) pure shear where $\langle\mathbf{F}\rangle=\lambda \mathbf{e}_{1} \otimes \mathbf{e}_{1}+\lambda^{-1} \mathbf{e}_{2} \otimes \mathbf{e}_{2}$ and (ii) simple shear where $\langle\mathbf{F}\rangle=\mathbf{I}+\gamma \mathbf{e}_{1} \otimes \mathbf{e}_{2}$ with $\lambda>0$ and $\gamma \geq 0$ denoting the applied stretch and amount of shear, respectively.

Here, it is important to remark that the periodic boundary condition (76) cannot be enforced, in general, when using elements with discontinuous pressure fields, such as the $\mathcal{M}_{2}-\mathcal{P}_{1}^{D}$, $\mathcal{M}_{1}-\mathcal{P}_{0}^{D}$, CPE4H, CPE6HM, and CPE8H elements. On the other hand, it can be enforced when using elements with continuous pressure fields, such as the $\mathcal{M}_{2}-\mathcal{M}_{1}$ elements. To gain insight into the effects of not enforcing the periodic boundary condition (76), Figs. 16 (a)(c) show plots of the macroscopic stress response for loading cases $(i)-(i i)$ against the applied stretch/shear making use of $\mathcal{M}_{2}-\mathcal{M}_{1}$ elements with and without the enforcement of (76). As it can be seen from the plots, the macroscopic stress from applying both periodic displacement and hydrostatic pressure boundary conditions are, for all practical purposes, identical to the ones from only applying the periodic displacement boundary conditions. Moreover, the unit cell considering only periodicity in the displacement field reaches a larger global stretch. Based on these observations, for the results presented in the remainder of this example, only periodic displacement boundary conditions are applied.

Figures 17 (a)-(d) depict the deformed configurations of the unit cell obtained with $\mathcal{M}_{2}-$ $\mathcal{M}_{1}, \mathcal{M}_{2}-\mathcal{P}_{1}^{D}, \mathcal{M}_{1}-\mathcal{P}_{0}^{D}, \mathrm{CPE} 4 \mathrm{H}, \mathrm{CPE} 6 \mathrm{MH}$, and CPE8H elements, at their respective maximum global stretches, $\lambda_{\max }=2.9132,2.6456,2.2515,1.9524,1.4308$, and 2.5861. The fringe scales correspond to the maximum principal stretches of each element, with those having a value of 8 and above being plotted as red. Similarly, Figs. 18 (a)-(c) depict the deformed configurations of the unit cell at the same level of global stretch $\lambda=2$ obtained with $\mathcal{M}_{2}-\mathcal{M}_{1}$, $\mathcal{M}_{2}-\mathcal{P}_{1}^{D}$, and $\mathcal{M}_{1}-\mathcal{P}_{0}^{D}$ elements, with the elements whose maximum principal stretches are larger than or equal to 5 plotted red. In addition, the relevant components of the macroscopic first Piola-Kirchhoff stresses $\langle\mathbf{P}\rangle$ as functions of the global stretch $\lambda$ or shear $\gamma$ are shown in Figs. 19 (a)-(c) for loadings (i)-(ii), and compared with the available analytical solution in the literature for elastomers reinforced by a random and isotropic distribution of polydisperse circular particles [64, 63]. 


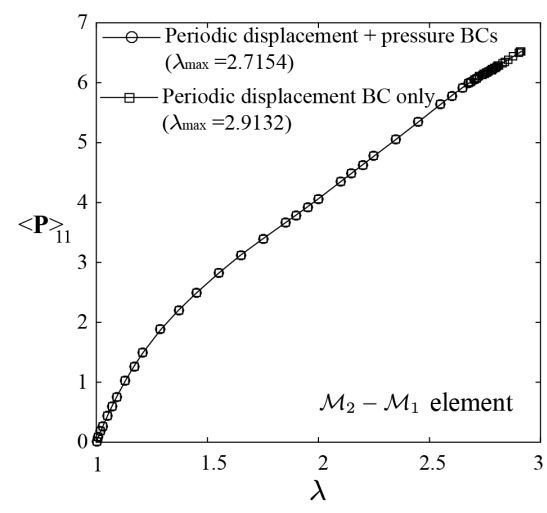

(a)

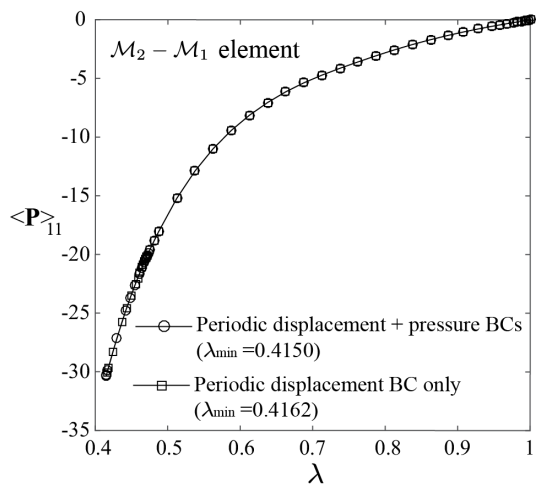

(b)

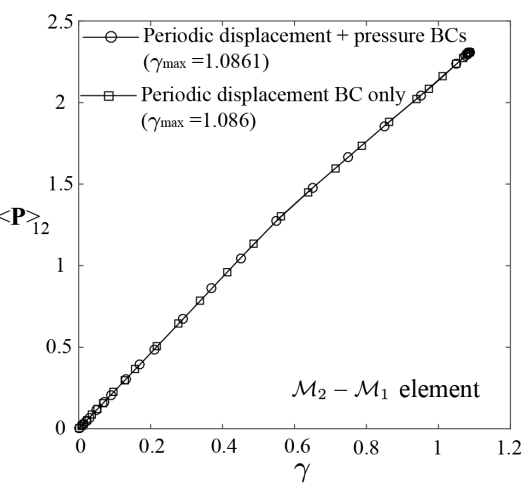

(c)

Figure 16: Plots of the macroscopic fist Piola-Kirchhoff stress as functions as the applied stretch/shear for the cases of applying both periodic displacement and pressure boundary conditions and applying only periodic displacement boundary condition under (a)-(b) pure and (c) simple shear. The plots are obtained using $\mathcal{M}_{2}-\mathcal{M}_{1}$ elements.

Several observations can be made from Figs. 17, 18, and 19. For starters, the macroscopic responses computed from all types of elements agree reasonably well with the analytical solution for all loading cases. Yet, the ones from quadrilateral and polygonal discretizations reach significantly larger global deformations than the ones from the triangular mesh. We note that, because of the high effective area fraction considered in this example $\left(c_{p}+c_{i}=36 \%\right)$ and the resulting high level of heterogeneity and localization in the deformation field, remeshing remedies would be of little help to make the unit cell with the triangular meshes (CPE6MH elements) stretch farther, as demonstrated in [65]. Comparing the polygonal meshes and quadrilateral meshes, except for the case of pure shear $(\lambda<1)$, where the quadrilateral mesh with CPE8H elements stretch farther, polygonal meshes can research larger deformation states than the quadrilateral ones, especially under pure shear $(\lambda>1)$ with $\mathcal{M}_{2}-\mathcal{M}_{1}$ and $\mathcal{M}_{1}-\mathcal{P}_{0}^{D}$ elements. Moreover, although all the polygonal elements produce almost identical deformed configurations at $\lambda=2$, as shown in Figs. 18 (a)-(c), the quadratic polygonal elements exhibit better performance than the linear ones in reaching a larger deformation state. As shown by Figs. 17 and 19 , the unit cell discretized with the $\mathcal{M}_{2}-\mathcal{M}_{1}$ and $\mathcal{M}_{2}-\mathcal{P}_{1}^{D}$ elements reach significantly larger global deformations than the one with the $\mathcal{M}_{1}-\mathcal{P}_{0}^{D}$ elements under pure shear. In addition, the linear polygonal elements produce slightly stiffer macroscopic behavior than quadratic polygonal elements. This is because the quadratic elements provide a richer functional approximation of displacements and so they can better represent the curvatures in the highly stretched regions (see, e.g., the red regions in Figs. 17 (a)-(c) and Figs. 18 (a)-(c)) than the linear ones. We also notice that although yielding almost identical macroscopic responses as the $\mathcal{M}_{2}-\mathcal{P}_{1}^{D}$ elements, the $\mathcal{M}_{2}-\mathcal{M}_{1}$ elements seem to be able to reach a slightly larger global stretch than $\mathcal{M}_{2}-\mathcal{P}_{1}^{D}$ elements under pure shear $(\lambda=2.91$ as compared to $\lambda=2.65)$. 


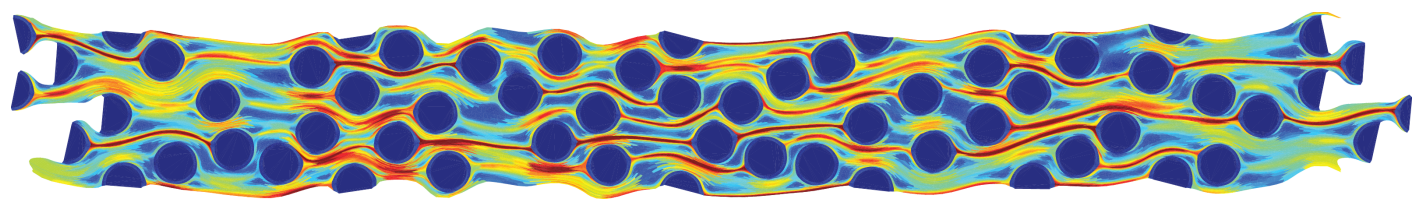

(a) $\mathcal{M}_{2}-\mathcal{M}_{1}$ element: $\lambda \max =2.9132$

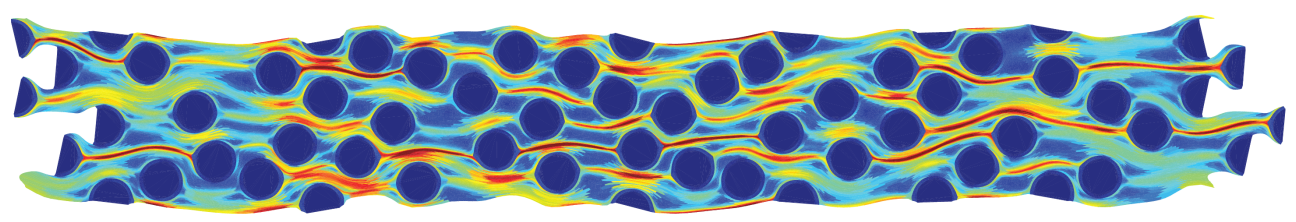

(b) $\mathcal{M}_{2}-\mathcal{P}_{1}^{D}$ element: $\lambda \max =2.6456$

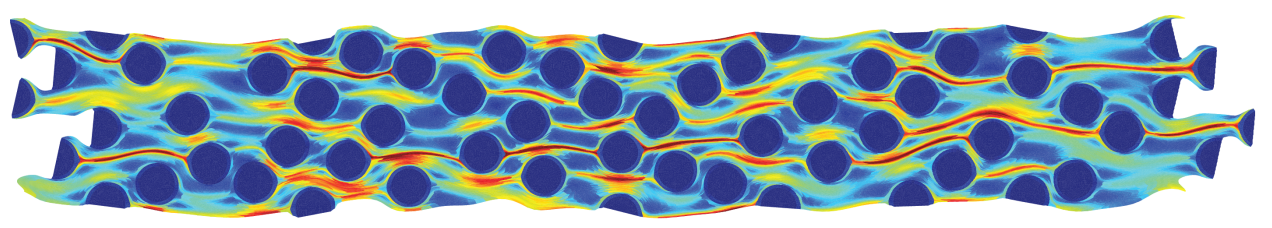

(c) CPE8H element: $\lambda \max =2.5861$

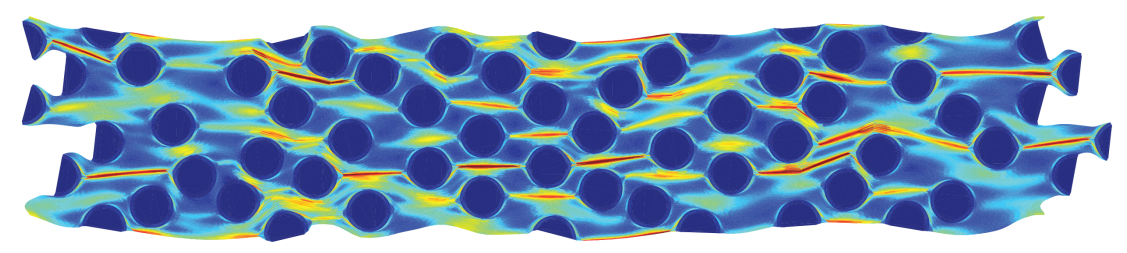

(d) $\mathcal{M}_{1}-\mathcal{P}_{0}^{D}$ element: $\lambda \max =2.2615$

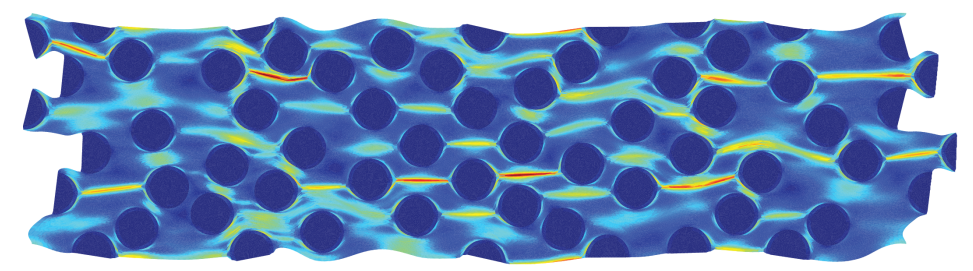

Max. Principle Stretch

(e) CPE8H element: $\lambda \max =1.9524$
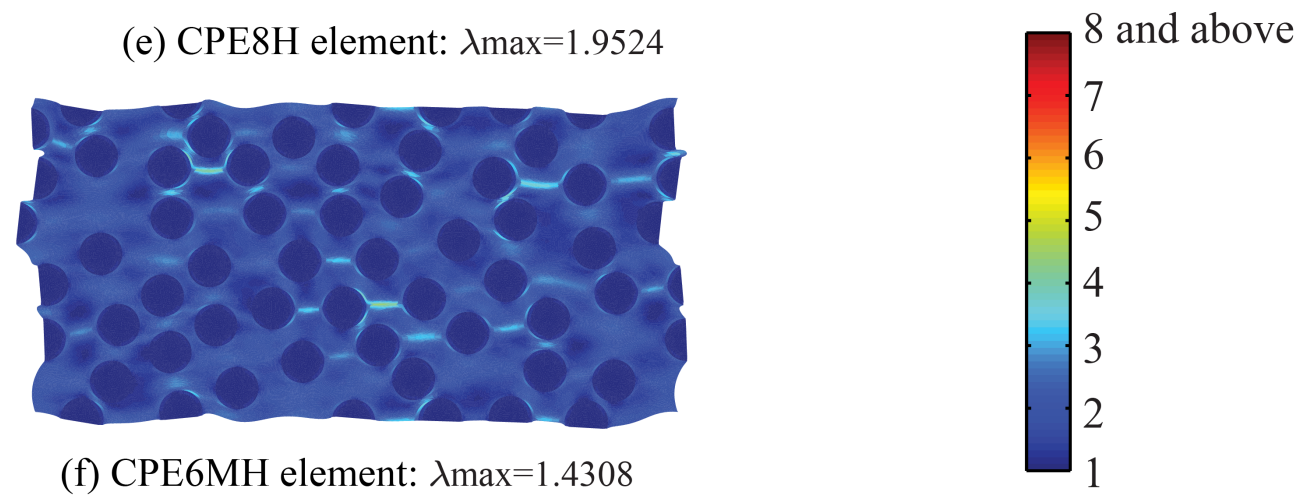

(f) CPE6MH element: $\lambda \max =1.4308$

(a)

Figure 17: The final deformed configuration reached by (a) $\mathcal{M}_{2}-\mathcal{M}_{1}$ elements, (b) $\mathcal{M}_{2}-\mathcal{P}_{1}$ elements, (c) CPE8H elements (solved in ABAQUS), (d) $\mathcal{M}_{1}-\mathcal{P}_{0}$ elements, (e) CPE4H elements (solved in ABAQUS), and (f) CPE6HM elements (solved in ABAQUS). 

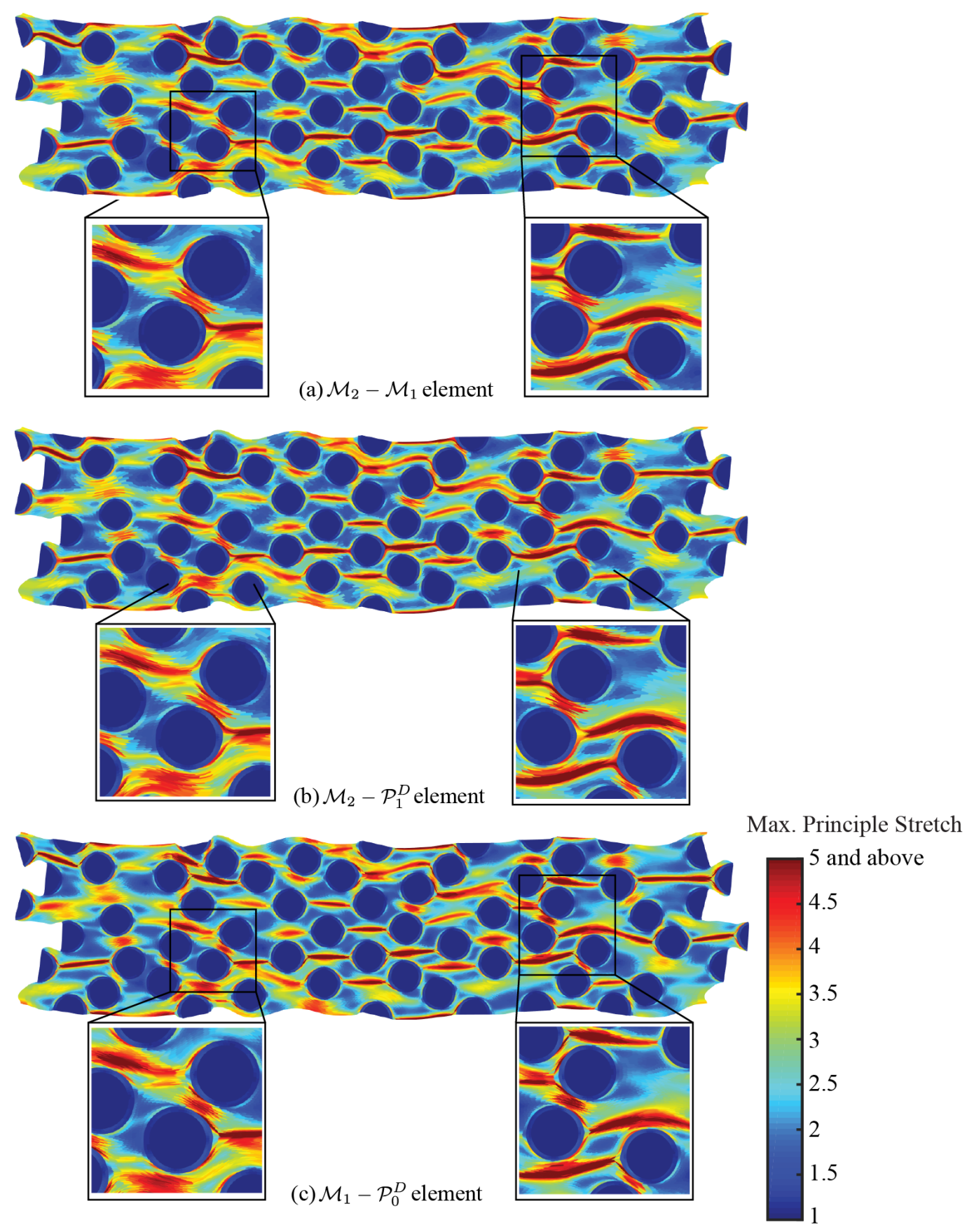

(a)

Figure 18: The deformed configuration at $\lambda=2$ obtained by (a) $\mathcal{M}_{2}-\mathcal{M}_{1}$ element, (b) $\mathcal{M}_{2}-\mathcal{P}_{1}^{D}$ element, and (c) $\mathcal{M}_{1}-\mathcal{P}_{0}^{D}$ element elements.

To further understand the performance of each of the four types of elements, we compute the error norm $\epsilon_{0, J}$ associated with the satisfaction of the incompressibility constraint. The results are summarized in Table 7. In essence, the linear mixed polygonal elements are seen to deliver results with larger errors than the quadratic ones. Furthermore, the results from the 


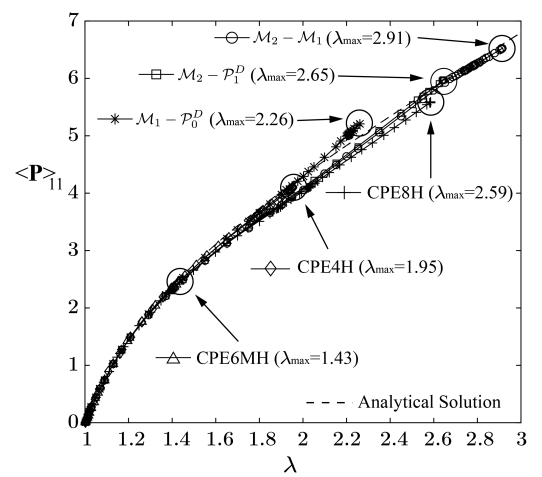

(a)

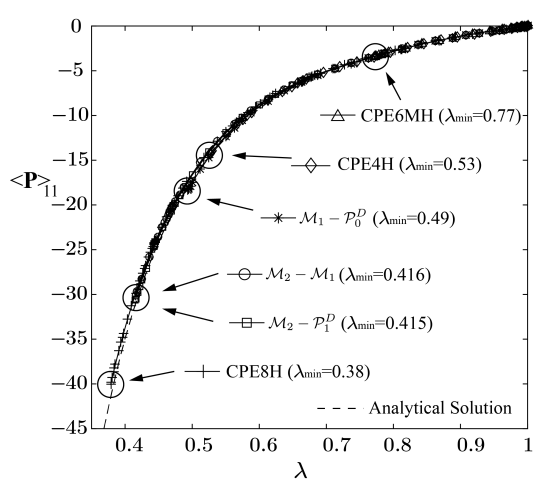

(b)

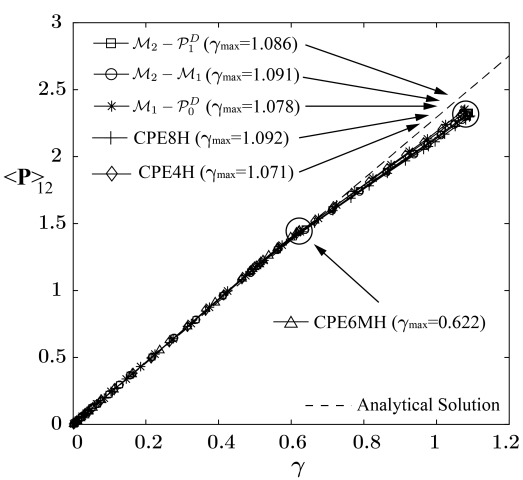

(c)

Figure 19: Plots of the macroscopic fist Piola-Kirchhoff stress as functions as the applied stretch/shear for different type of elements under (a) pure shear $(\lambda \geq 1)$, (b) pure shear $(0<\lambda<1)$ and (c) simple shear.

$\mathcal{M}_{2}-\mathcal{P}_{1}^{D}$ elements are more accurate in satisfying the incompressibility constraint than the $\mathcal{M}_{2}-\mathcal{M}_{1}$ elements on the same mesh, which is a consequence of the larger number of global pressure DOFs that the $\mathcal{M}_{2}-\mathcal{P}_{1}^{D}$ mesh contains. As a result, the $\mathcal{M}_{2}-\mathcal{M}_{1}$ elements are able to reach a larger global stretch than the $\mathcal{M}_{2}-\mathcal{P}_{1}^{D}$ elements under pure shear.

Table 7: Error in the approximation of the incompressibility constraint under different loading conditions

\begin{tabular}{c|cccc}
\hline \hline & \# of Pressure DOFs & $\epsilon_{0, J}(\lambda=2)$ & $\epsilon_{0, J}(\lambda=0.6)$ & $\epsilon_{0, J}(\gamma=0.6)$ \\
\hline $\mathcal{M}_{1}-\mathcal{P}_{0}^{D}$ elements & 39738 & 0.1180 & 0.0757 & 0.0355 \\
$\mathcal{M}_{2}-\mathcal{P}_{1}^{D}$ elements & 60615 & 0.0295 & 0.0163 & 0.0071 \\
$\mathcal{M}_{2}-\mathcal{M}_{1}$ elements & 37495 & 0.0508 & 0.0341 & 0.0177 \\
\hline
\end{tabular}

\subsection{Onset of cavitation in fiber reinforced elastomers}

An interesting phenomenon of re-emerging interest that occurs in elastomers is the so-called phenomenon of cavitation, namely, the sudden growth of pre-existing defects into large enclosed cavities under sufficiently large loads [66]. Cho and Gent [67] studied this phenomenon in fiber reinforced elastomers by means of some ingenious experiments. As shown in Fig. 20 (a), they fabricated specimens consisting of two steel tubes of length $L$ and diameter $D$, parallely aligned 
at an initial distance $d$, bonded by a layer of transparent silicone rubber. The surfaces of the steel tubes were polished and treated with a primer so as to prevent debonding. The specimens were subjected to uniaxial tension transverse to the tubes. The onset of cavitation could then be monitored visually through the transparent rubber.

In this subsection, motivated by the work of Lefèvre et al. [68], we reproduce theoretically (via a 2D plane-strain model) two of the experiments of Cho and Gent [67] by means of the polygonal finite element framework presented in this paper. Among the several sets of experiments that they reported, we choose two with dimensions given in Table 8. In the table, we also include the values of the measured "macroscopic" strain $\bar{\varepsilon}_{e}$ at which cavitation occurred. In addition, Fig. 20 (d) shows a photograph of the cavity formed between the two steel tubes in the specimen with initial distance $d=0.43 \mathrm{~mm}$.

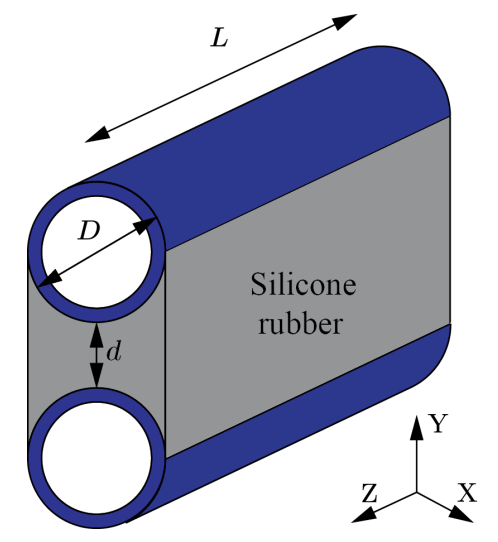

(a)

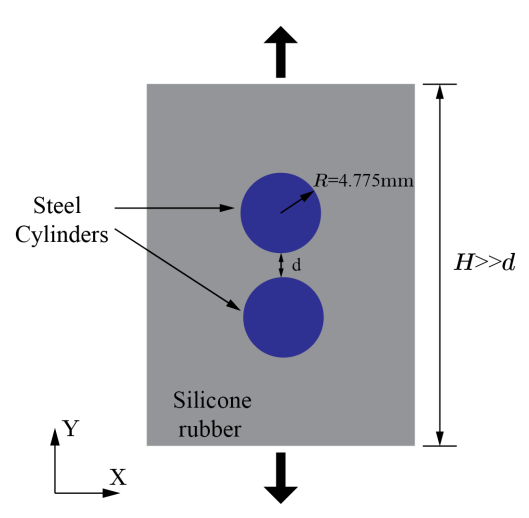

(b)

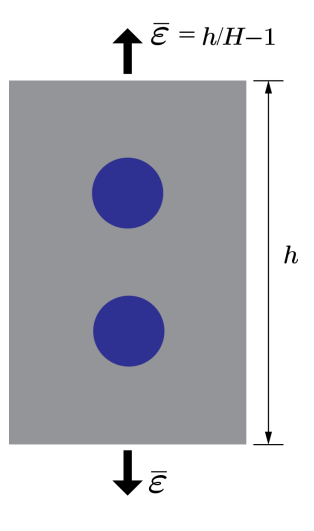

(c)

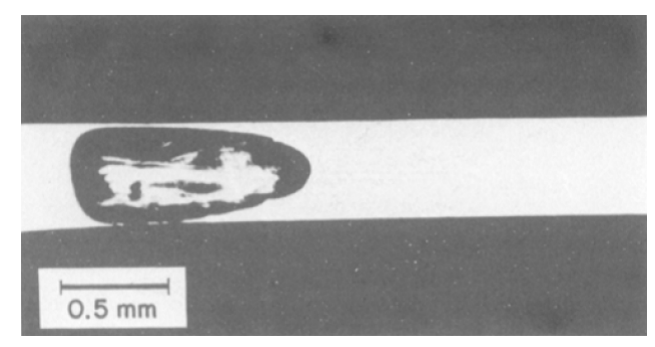

(d)

Figure 20: (a) Schematic of the experimental setup of Cho and Gent [67]. (b) A two-dimensional plane-strain model of the experiment in the undeformed configuration. (c)A two-dimensional plane-strain model of the experiment in the deformed configuration. (d) A photograph of the lateral view (X direction) of the cavity formed between the two steel tubes for the case of $d=0.43 \mathrm{~mm}[67]$.

Given that the length of the steel tubes $L=12.5 \mathrm{~mm}$ is more than one order of magnitude larger than their initial distance $d$ for both cases $(d=0.43 \mathrm{~mm}$ and $0.91 \mathrm{~mm})$, and that the cavities observed in the experiments are somewhat cylindrical in shape, as shown by Fig. 20 (d), we idealize the experiment as a two-dimensional plane-strain problem and restrict attention to the growth of cylindrical defects. More specifically, we consider a square block of side $H=300$ 
Table 8: Summary of the experimental results by Cho and Gent [67] with different initial distance between two steel tubes

\begin{tabular}{c|c|c|c|c|c|c}
\hline \hline Specimen No. & $L(\mathrm{~mm})$ & $D(\mathrm{~mm})$ & $d(\mathrm{~mm})$ & $d / D$ & $\delta(\mathrm{mm})$ & $\bar{\varepsilon}_{e}$ \\
\hline$\# 1$ & 12.5 & 9.5 & 0.43 & 0.045 & 0.034 & 0.08 \\
$\# 2$ & 12.5 & 9.5 & 0.91 & 0.095 & 0.255 & 0.28 \\
\hline
\end{tabular}

$\mathrm{mm}$ in which two cylindrical fibers are embedded adjacent to each other. The size of the block is large enough to avoid the interaction between the fibers and the outer boundary. The block is subjected to uniaxial tension in the direction of alignment of the fibers. As in the experiments, the "macroscopic" strain is defined as $\bar{\varepsilon} \doteq h / H-1$, where $h$ is the deformed length of the block in the direction of applied tension. Figures 20 (b) and (c) show schematic descriptions of the model in its undeformed and deformed configurations. The silicone rubber is modeled as a one-term compressible Lopez-Pamies material [69]:

$$
W(\mathbf{F})=\frac{3^{1-\alpha}}{2 \alpha} \mu\left[(\mathbf{F}: \mathbf{F})^{\alpha}-3^{\alpha}\right]-\mu(\operatorname{det} \mathbf{F}-1)+\left(\frac{\mu}{2}+\frac{\kappa}{2}-\frac{\alpha \mu}{3}\right)(\operatorname{det} \mathbf{F}-1)^{2} .
$$

Its Legendre transformation $\widehat{W}^{*}(\mathbf{F}, \widehat{q})$ is given by:

$$
\widehat{W}^{*}(\mathbf{F}, \widehat{q})=-\frac{3^{1-\alpha}}{2 \alpha} \mu\left[(\mathbf{F}: \mathbf{F})^{\alpha}-3^{\alpha}\right]+\frac{(\mu+\widehat{q})^{2}}{2\left(\mu+\kappa-\frac{2}{3} \alpha \mu\right)} .
$$

The shear modulus $\mu$ of the rubber is taken to be 1 and, to reasonably model the nearly incompressible behavior of the silicone rubber, its bulk modulus $\kappa$ is taken to be 1000 . The material parameter $\alpha$ is taken to be 0.6 here in order to ensure appropriate growth conditions that allow for cavitation in 2D [70]. Moreover, again, the fibers are treated as infinitely rigid bodies (since the shear modulus of the steel is in the order of $80 \mathrm{GPa}$ ) that are perfectly bonded to the rubber. In all of the calculations for this example problem, we employ the $\mathbf{F}$-formulation of Chi et al. [3].

From a computational point of view, the modeling of cavitation is challenging with standard finite elements because of the extreme local deformations that take place around the boundaries of the growing defects $[71,72]$. In the literature, use has been made of structured quadrilateral distretizations which have proved successful in a variety of problems (see, e.g., [72, 68]). However, such an approach is not deemed viable to deal with material systems with realistically complex microstructures. Unstructured polygonal discretizations, on the other hand, have recently shown potential as a viable approach [3]. To further examine the performance of different types of mixed polygonal elements for cavitation problems, we consider unstructured polygonal discretizations and consider all three types of mixed polygonal elements in this example.

Following the analysis of Lefèvre et al. [68], we begin by monitoring the onset of cavitation 
for the two cases with initial fiber distances $d=0.41$ and $0.91 \mathrm{~mm}$. Although the actual growth of defects in rubber is a complex process that may involve irreversible inelastic deformations (e.g., fracture), cavitation criteria based on purely elastic deformations have been shown to provide accurate qualitative agreement with experimental observations, suggesting that the elastic behavior of rubber plays a key role in the occurrence of the phenomenon [68]. For a review of those criteria, the interested reader is referred to the papers by Lopez-Pamies et al. [73, 74]. For the problem under investigation here, we make use of the cavitation criterion derived by Lopez-Pamies [75] for compressible isotropic solids under general planestrain loading conditions. The criterion states that, under plane-strain conditions, defects inside a compressible isotropic solid may suddenly grow into cylindrical cavities whenever the principal stretches $\left(\lambda_{1}, \lambda_{2}\right)$ satisfy the following condition:

$$
\frac{\frac{\partial \Phi}{\partial \lambda_{1}}\left(\lambda_{1}, \lambda_{2}\right)-\frac{\partial \Phi}{\partial \lambda_{2}}\left(\lambda_{1}, \lambda_{2}\right)}{\lambda_{1}-\lambda_{2}}=0
$$

where $\Phi\left(\lambda_{1}, \lambda_{2}\right)$ stands for the stored-energy function, written in terms of the in-plane principal stretches, characterizing the elastic response of the rubber under plane-strain conditions. Upon recognizing that $\mathbf{F}: \mathbf{F}=\lambda_{1}^{2}+\lambda_{2}^{2}+1$ and $\operatorname{det} \mathbf{F}=\lambda_{1} \lambda_{2}$, the criterion (79) reduces to

$$
\kappa+2 \mu-\frac{2 \alpha \mu}{3}+3^{1-\alpha} \mu\left(\lambda_{1}^{2}+\lambda_{2}^{2}+1\right)^{(\alpha-1)}-\lambda_{1} \lambda_{2}\left(\kappa+\mu-\frac{2 \alpha \mu}{3}\right)=0
$$

when specialized to the stored-energy function (77).

To monitor the onset of cavitation criterion (80), we make use of a polygonal mesh with $30000 \mathcal{M}_{1}-\mathcal{P}_{0}^{D}$ elements, which are graded so that smaller elements are placed in between the fibers [58]. Figures 21 shows the mesh utilized for the case with $d=0.43 \mathrm{~mm}$. Figures 22

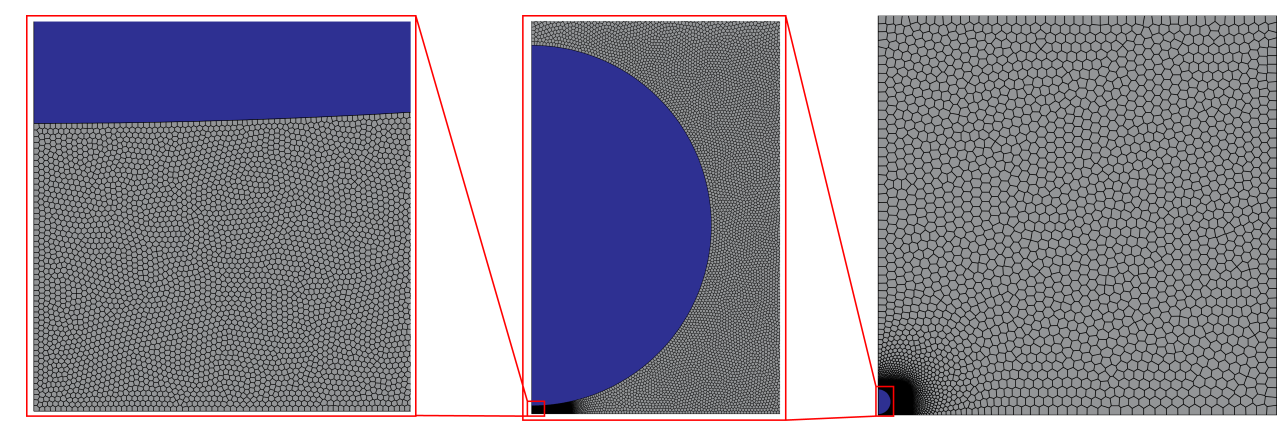

Figure 21: he polygonal mesh used of the model of initial distance $d=0.41 \mathrm{~mm}$, which consists of 30000 polygonal elements.

(a) and (b) depict the deformed states of the region between the two rigid fibers with initial distances $d=0.41 \mathrm{~mm}$ and $0.91 \mathrm{~mm}$ at four levels of the applied macroscopic strain. In the 
figures, those elements whose average in-plane principal stretches satisfy the criterion (80) are plotted in red, indicating the possible onset of cavitation at the material points that they sit on. We emphasize that except for the elements plotted, no other elements are found to satisfy the cavitation criterion during the entire loading process. For both cases of $d=0.41 \mathrm{~mm}$ and 0.91 $\mathrm{mm}$, the criterion is first satisfied at the the rubber/fiber interfaces. As the macroscopic strain increases, the satisfaction of the criterion quickly propagates to the midpoint between the fibers and then continues to grow outward. For the case of larger interfiber distance, $d=0.91 \mathrm{~mm}$, the onset of cavitation at the rubber/fiber interfaces occurs at a significantly larger macroscopic strain, $\bar{\varepsilon} \approx 8.835 \%$, as compared to $\bar{\varepsilon} \approx 5.66 \%$. Similarly, the onset of cavitation at the midpoint between the fiber also occurs at a considerably larger macroscopic strain, $\bar{\varepsilon} \approx 9.635 \%$, for the case of $d=0.91 \mathrm{~mm}$, as compared to $\bar{\varepsilon} \approx 5.86 \%$ for the case of $d=0.43 \mathrm{~mm}$. Qualitatively, these relative values are in fair agreement with the reported experimental data summarized in Table 8 .

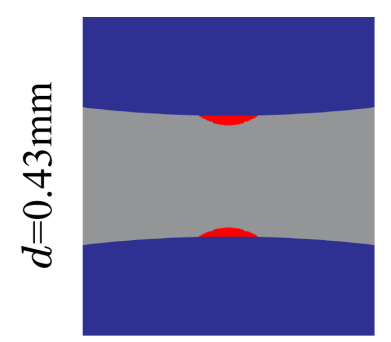

$\bar{\varepsilon}=5.66 \%$

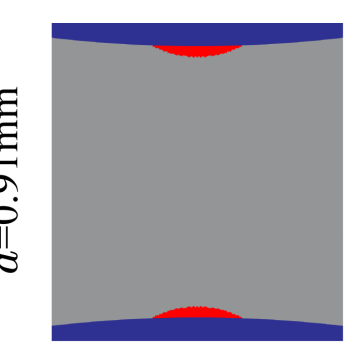

$\bar{\varepsilon}=8.835 \%$

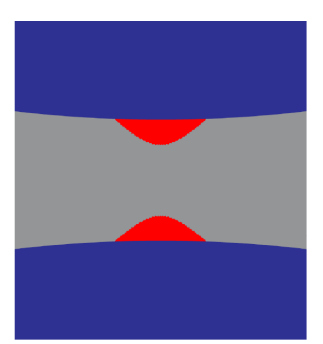

$\bar{\varepsilon}=5.76 \%$

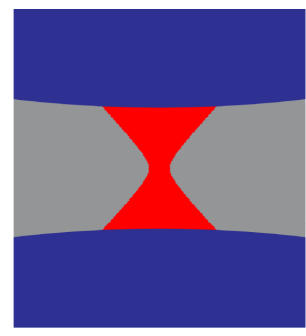

$\bar{\varepsilon}=5.86 \%$

(a)

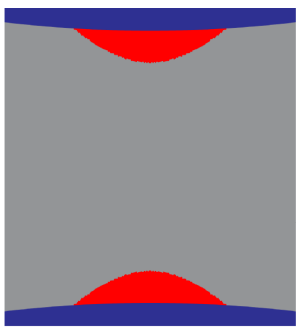

$\bar{\varepsilon}=9.085 \%$

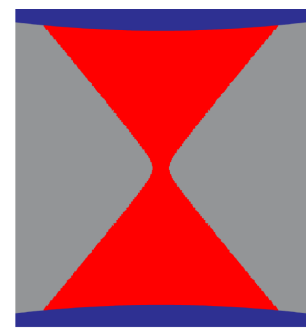

$\bar{\varepsilon}=9.635 \%$

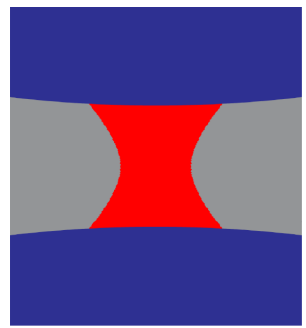

$\bar{\varepsilon}=5.96 \%$

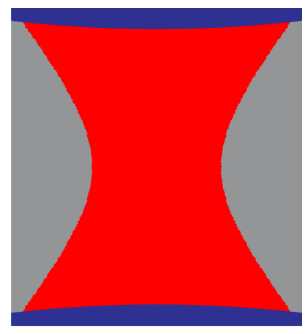

$\bar{\varepsilon}=9.935 \%$

(b)

Figure 22: The deformed configurations of the region between two fibers under four levels of macroscopic strains for cases (a) $d=0.43 \mathrm{~mm}$ and (b) $d=0.91 \mathrm{~mm}$. The material points at which the cavitation criterion (80) are satisfied are plotted in red.

With the knowledge that the onset of cavitation might first occur at the rubber/fiber interfaces on the inner sides of the fibers and then propagates to the midpoint between the fibers, we proceed to introduce pre-existing defects at those locations in the finite element models to study their actual growth and interaction. Thus, three vacuous defects that are circular in 
shape are introduced in each finite element model with an initial radius $R_{c}=2 \mu \mathrm{m}$. Two of them are placed $1 \mu \mathrm{m}$ away from the inner side of each fiber at the rubber/fiber interfaces while the third one is placed at the midpoint between the fibers. Both linear and quadratic polygonal meshes are considered, containing 25000 and 10000 elements respectively. As an example, Fig. 23 (a) shows the linear polygonal mesh utilized in the case with the initial distance $d=0.43 \mathrm{~mm}$ [58]. We also consider structured quadrilateral meshes of ABAQUS CPE4H elements to serve as reference solutions [68], which are structured radially along each defect to accommodate its growth. Figure 23 (b) shows the structured quadrilateral mesh utilized in the case with the initial distance $d=0.43 \mathrm{~mm}$

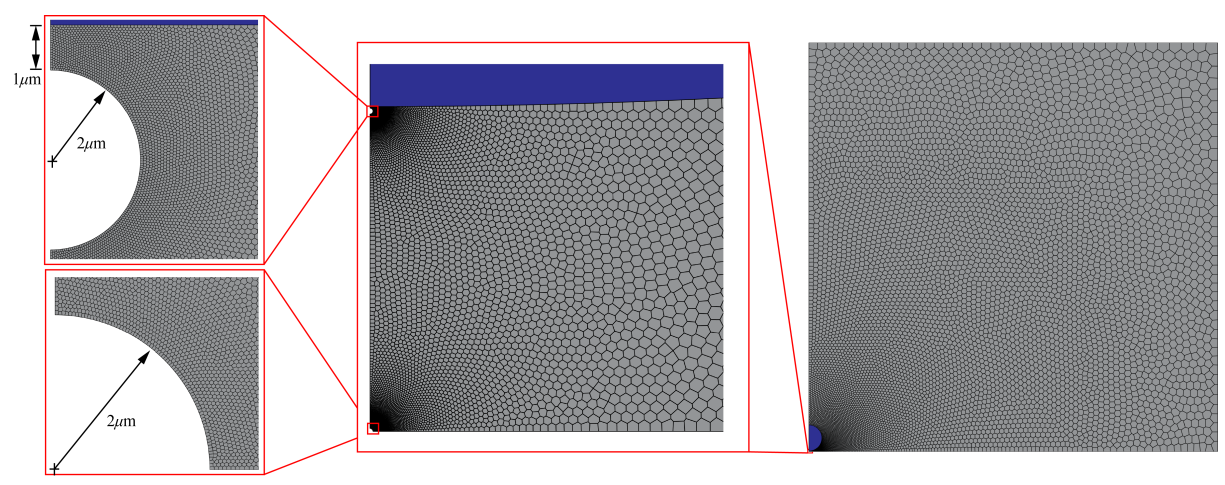

(a)
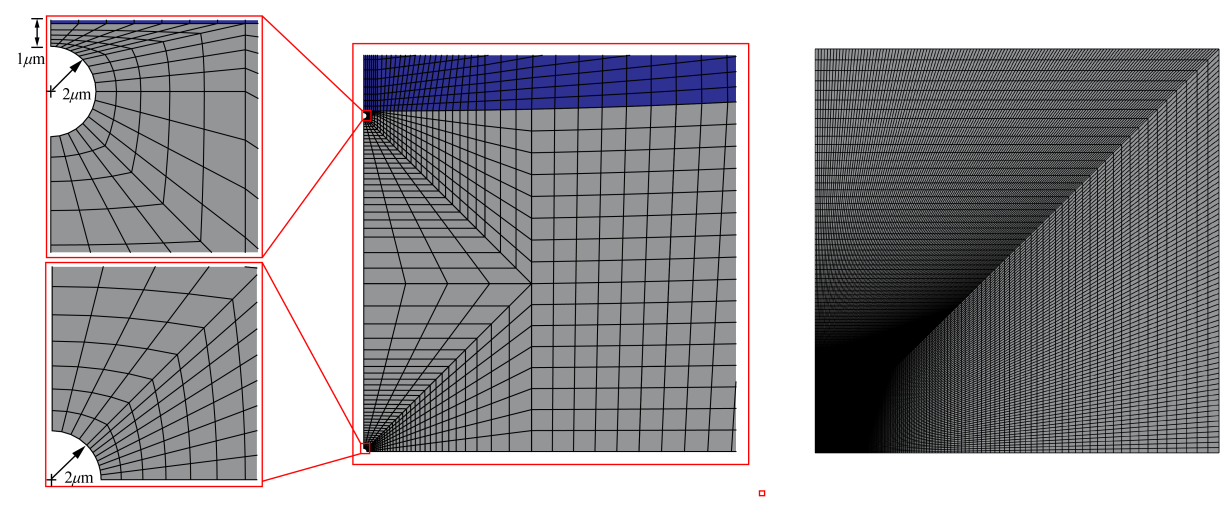

(d)

Figure 23: (a) The linear polygonal mesh used for the model of initial distance $d=0.41 \mathrm{~mm}$ containing three defects. The mesh consists of 25000 elements. (b) The quadrilateral mesh used of the model of initial distance $d=0.41 \mathrm{~mm}$, which consists of 40843 linear quadrilateral elements.

Figures 24 (a) and (b) show snapshots of deformed states of the regions between the fibers obtained by $\mathcal{M}_{1}-\mathcal{P}_{0}^{D}$ elements for cases with initial distances $d=0.43 \mathrm{~mm}$ and $0.91 \mathrm{~mm}$, respectively, at four stages of macroscopic strains, $\bar{\varepsilon}=6.39,9.89,14.89$, and $30.39 \%$. The results obtained by quadratic mixed polygonal elements are identical and therefore are not shown here. To gain more quantitative insight, we also quantify the current-to-undeformed area ratios $a^{i} / A^{i}$ and $a^{m} / A^{m}$ of the defects at the interfaces and midpoints. The results are displayed in Figs. 
25 (a) and (b) as functions of the applied macroscopic strain $\bar{\varepsilon}$. For comparison purposes, these figures include results computed with a structured quadrilateral mesh of ABAQUS CPE4H elements [68]. An immediate observation is that all the mixed polygonal elements, namely, the $\mathcal{M}_{1}-\mathcal{P}_{0}^{D}, \mathcal{M}_{2}-\mathcal{P}_{1}^{D}$, and $\mathcal{M}_{2}-\mathcal{M}_{1}$ elements are able to capture the entire growth history and interactions between the defects and agree with the ABAQUS results. Physically, the results indicate that the defects at the interfaces grow indeed first but that, upon further loading, the defects at the midpoint start to grow as well. Further loading results in the contraction of the interfacial defects and the concentration of all of the growth in the midpoint defects, which eventually become visible cavities. This intricate behavior appears to be in qualitative agreement with the experiments of Cho and Gent, at least for the specimen with initial interfiber separation $d=0.43 \mathrm{~mm}$. The reason behind such a behavior is that the midpoint is the farthest point from the stiff rubber/fiber interface and therefore is the location where the growth of a cavity is the least energetically expensive [68]. Furthermore, if we heuristically define that cavitation occurs whenever a defect grows 1000 times in area say, the specimen with initial fiber distance of $d=0.43 \mathrm{~mm}$ cavitates at a macroscopic strain around $\bar{\varepsilon} \approx 6 \%$ while the one with initial fiber distance of $d=0.91 \mathrm{~mm}$ cavitates at around $\bar{\varepsilon} \approx 10 \%$. These values are in good agreement with the prediction given by the criterion (80) as well as with the experimental observations. We emphasize that such an agreement is remarkable given that the computations carried out here account only for the elastic deformation of the defects, whereas in the experiments inelastic growth of defects by fracture does occur.

\section{Conclusion}

The modeling of nonlinear elastic materials with standard finite elements under finite deformations is a challenging task due to, by and large, the invariably large local deformations induced by their heterogeneity. Recently, lower order polygonal elements have shown potential in modeling such a class of materials. To further examine the capabilities of polygonal elements to model nonlinear elastic materials, this paper has generalized existing displacement-based and mixed polygonal elements to higher order and has provided a study of their performance under finite deformations. One critical aspect of the generalization is the numerical integration scheme. Because polygonal elements contain functions of non-polynomial nature in their finite element spaces, they typically suffer from consistent errors with the existing integrations schemes. As shown by numerical tests here, the consistent errors do not vanish under mesh refinement when a standard integration scheme is used, which lead to non-convergent finite element results. This problems becomes even more severe when using higher order elements. To address this issue, this paper has proposed to employ a gradient correction scheme which can be applied to both displacement-based and mixed polygonal elements of arbitrary order with any numerical integration scheme that satisfy the corresponding minimal order requirement. 

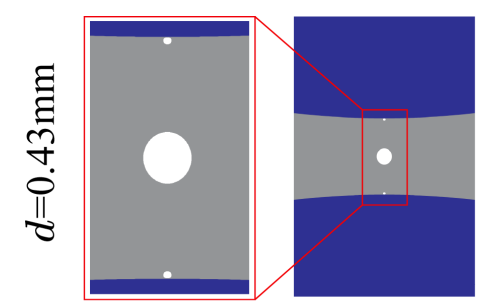

$\bar{\varepsilon}=6.39 \%$

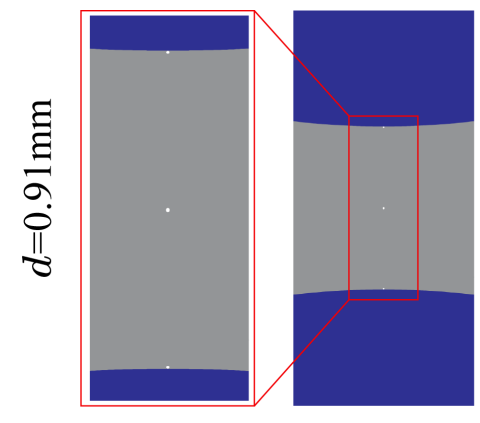

$\bar{\varepsilon}=6.39 \%$ (a)

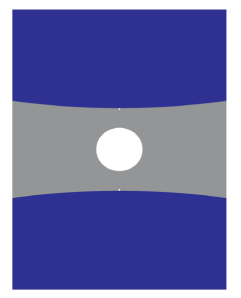

$$
\bar{\varepsilon}=9.89 \%
$$

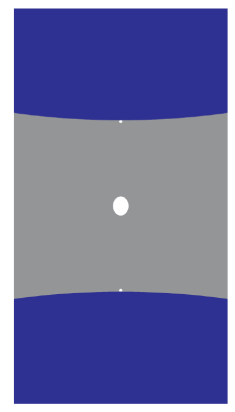

$\bar{\varepsilon}=9.89 \%$

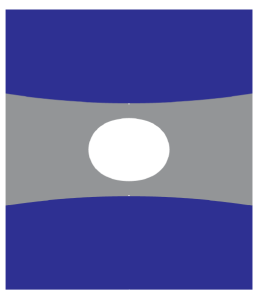

$\bar{\varepsilon}=14.89 \%$

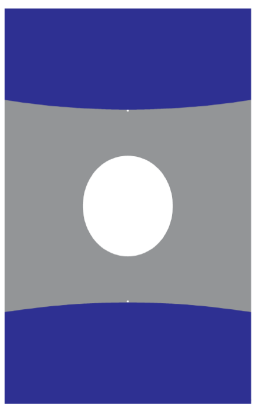

$\bar{\varepsilon}=14.89 \%$

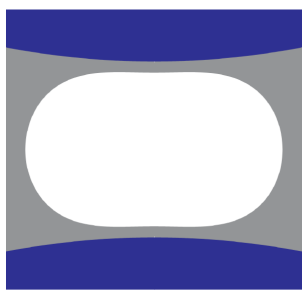

$\bar{\varepsilon}=30.39 \%$

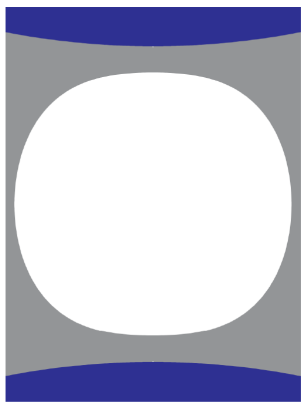

$\bar{\varepsilon}=30.39 \%$

(b)

Figure 24: With three defects introduced in the model, the deformed configurations of the region between two fibers under four levels of macroscopic strains for cases (a) $d=0.43 \mathrm{~mm}$ and (b) $d=0.91 \mathrm{~mm}$.

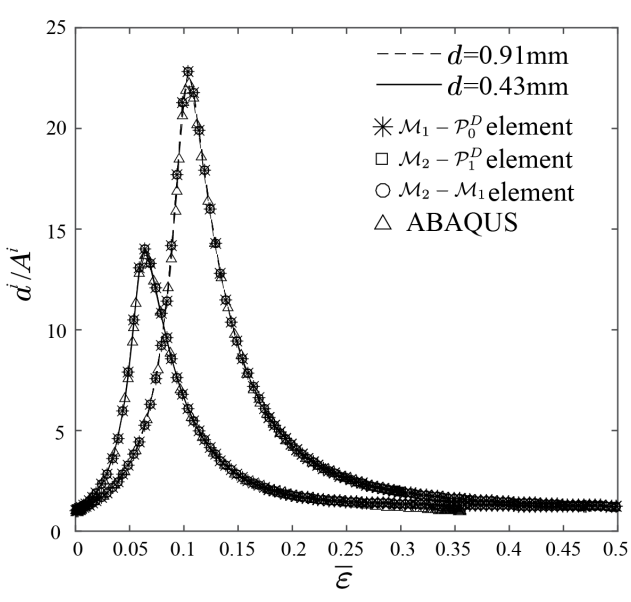

(a)

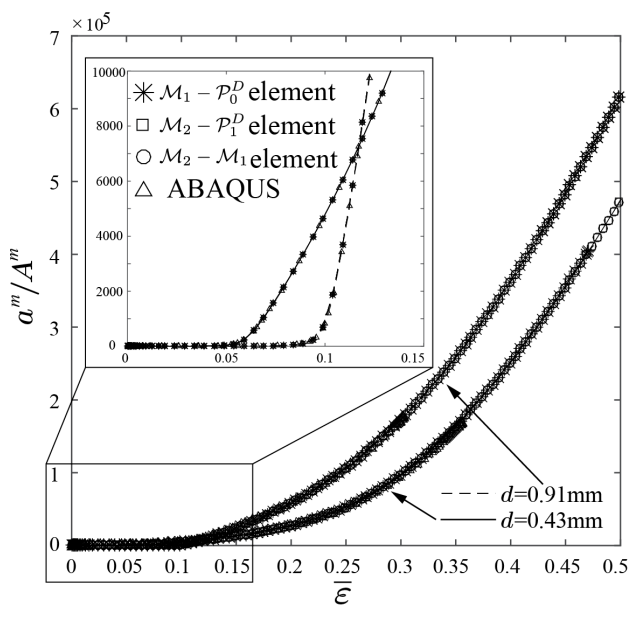

(b)

Figure 25: The growth histories of the three defects quantified by the ratio of their deformed areas to their initial areas as functions of the applied macroscopic strain $\varepsilon$ for both cases of $d=0.43 \mathrm{~mm}$ and $d=0.91 \mathrm{~mm}$ :

(a) the defects on the matrix/fiber interface, and (b) the defect in the middle of the two fibers.

With the correction scheme, both linear and quadratic polygonal elements have been shown to yield optimally convergent results with displacement-based and mixed approximations. Furthermore, two choices of the pressure field approximations have been discussed for the mixed 
polygonal elements, namely, the discontinuous approximation and continuous approximation, and their performances on numerical stability and accuracy have been investigated. Our numerical studies indicate that both families of mixed polygonal elements are numerically stable in linear elasticity problems without any additional stabilization treatments up to quadratic order. The comparison of performance in the accuracy (for instance, errors in satisfaction of incompressibility constraint) between the different types of mixed polygonal element, on the other hand, appears to be problem specific (c.f., the discussions in the examples of bending of a rectangular block into a semicircle and particle reinforced elastomer), and thus needs further study. For demonstration purposes, we have employed the proposed polygonal elements to model the nonlinear response of particle reinforced elastomers with interphases and the onset of cavitation in fiber reinforced elastomers. These challenging physically-based applications have indicated that the proposed polygonal elements are well suited to deal with complex microstructures including particulate microstructures and those involving different length scales, and appears to be more tolerant to large local deformation than the standard finite elements (triangles and quads).

\section{Acknowledgement}

We acknowledge the support from the US National Science Foundation (NSF) through grant CMMI \#1437535. The information presented in this paper is the sole opinion of the authors and does not necessarily reflect the views of the sponsoring agency. 


\section{References}

[1] C. Talischi, G. H. Paulino, Addressing integration error for polygonal finite elements through polynomial projections: A patch test connection, Mathematical Models and Methods in Applied Sciences 24 (08) (2014) 1701-1727.

[2] G.Manzini, A.Russo, N.Sukumar, New perspective on polygonal and polyhedral finite element method, Mathematical Models and Methods in Applied Sciences 24 (08) (2014) 1665-1699.

[3] H. Chi, C. Talischi, O. Lopez-Pamies, G. H. Paulino, Polygonal finite elements for finite elasticity, International journal for numerical methods in engineering 101 (2015) 305-328.

[4] C. Talischi, A. Pereira, I. Menezes, G. H. Paulino, Gradient correction for polygonal and polyhedral finite elements, International Journal for Numerical Methods in Engineering $102(3-4)(2015) 728-747$.

[5] L. Beirão Da Veiga, G. Manzini, A Virtual Element Method with arbitrary regularity, IMA Journal on Numerical Analysis doi: 10.1093/imanum/drt018.

[6] A. L. Gain, C. Talischi, G. H. Paulino, On the virtual element method for three-dimensional linear elasticity problems on arbitrary polyhedral meshes, Computer Methods in Applied Mechanics and Engineering 282 (2014) 132-160.

[7] F. Brezzi, R. Falk, L. Donatella Marini, Basic principles of mixed virtual element methods, ESAIM: Mathematical Modelling and Numerical Analysis 48 (04) (2014) 1227-1240.

[8] Y. Krongauz, T. Belytschko, Consistent pseudo-derivatives in meshless methods, Computer methods in applied mechanics and engineering 146 (3) (1997) 371-386.

[9] J. E. Bishop, Simulating the pervasive fracture of materials and structures using randomly close packed Voronoi tessellations., Computational Mechanics 44 (4) (2009) 455-471.

[10] J. Bishop, A displacement-based finite element formulation for general polyhedra using harmonic shape functions., International Journal for Numerical Methods in Engineering 97 (1) (2014) 1-31.

[11] D. Boffi, F. Brezzi, M. Fortin, Mixed finite element methods and applications, Springer, 2013.

[12] J. Oden, Recent developments in the theory of finite element approximations of boundary value problems in nonlinear elasticity, Computer Methods in Applied Mechanics and Engineering 17 (1979) 183-202. 
[13] P. Le Tallec, Existence and approximation results for nonlinear mixed problems: application to incompressible finite elasticity, Numerische Mathematik 38 (3) (1982) 365-382.

[14] L. Beirão Da Veiga, K. Lipnikov, A mimetic discretization of the Stokes problem with selected edge bubbles, SIAM J Sci Comput 32 (2) (2010) 875-893.

[15] T. J. R. Hughes, The finite element method: linear static and dynamic finite element analysis, Courier Dover Publications, 2012.

[16] D. Chapelle, K. J. Bathe, The inf-sup test, Computers \& structures 47 (4) (1993) 537-545.

[17] J. T. Oden, N. Kikuchi, Finite element methods for constrained problems in elasticity, International Journal for Numerical Methods in Engineering 18 (5) (1982) 701-725.

[18] J. Chen, C. Pan, T. Chang, On the control of pressure oscillation in bilinear-displacement constant-pressure element, Computer methods in applied mechanics and engineering 128 (1) (1995) 137-152.

[19] T. Hughes, L. Franca, M. Balestra, A new finite element formulation for computational fluid dynamics: V. circumventing the babuška-brezzi condition: A stable petrov-galerkin formulation of the stokes problem accommodating equal-order interpolations, Computer Methods in Applied Mechanics and Engineering 59 (1) (1986) 85-99.

[20] F. Brezzi, J. Douglas Jr, Stabilized mixed methods for the stokes problem, Numerische Mathematik 53 (1-2) (1988) 225-235.

[21] C. Talischi, A. Pereira, G. H. Paulino, I. F. M. Menezes, M. S. Carvalho, Polygonal finite elements for incompressible fluid flow, International Journal for Numerical Methods in Fluids 74 (2014) 134-151.

[22] A. Rand, A. Gillette, C. Bajaj, Quadratic serendipity finite elements on polygons using generalized barycentric coordinates, Mathematics of computation 83 (290) (2014) 26912716.

[23] N. Sukumar, Quadratic maximum-entropy serendipity shape functions for arbitrary planar polygons, Computer Methods in Applied Mechanics and Engineering 263 (2013) 27-41.

[24] R. W. Ogden, Nonlinear elastic deformations, Courier Dover Publications, 1997.

[25] J. E. Marsden, T. Hughes, Mathematical foundations of elasticity, Courier Corporation, 1994.

[26] P. Wriggers, Nonlinear finite element methods, Vol. 4, Springer, 2008. 
[27] J. Bonet, R. D. Wood, Nonlinear continuum mechanics for finite element analysis, Cambridge university press, 2008.

[28] L. R. Herrmann, Elasticity equations for incompressible and nearly incompressible materials by a variational theorem., AIAA journal 3 (10) (1965) 1896-1900.

[29] S. N. Atluri, E. Reissner, On the formulation of variational theorems involving volume constraints, Computational Mechanics 5 (5) (1989) 337-344.

[30] J. C. Simo, R. L. Taylor, K. S. Pister, Variational and projection methods for the volume constraint in finite deformation elasto-plasticity, Computer Methods in Applied Mechanics and Engineering 51 (1) (1985) 177-208.

[31] T. Sussman, K. J. Bathe, A finite element formulation for nonlinear incompressible elastic and inelastic analysis, Computers \& Structures 26 (1) (1987) 357-409.

[32] U. Brink, E. Stein, On some mixed finite element methods for incompressible and nearly incompressible finite elasticity, Computational Mechanics 19 (1) (1996) 105-119.

[33] T. Y. P. Chang, A. F. Saleeb, G. Li, Large strain analysis of rubber-like materials based on a perturbed lagrangian variational principle, Computational mechanics 8 (4) (1991) 221-233.

[34] J. S. Chen, W. Han, C. T. Wu, W. Duan, On the perturbed lagrangian formulation for nearly incompressible and incompressible hyperelasticity, Computer methods in applied mechanics and engineering 142 (3) (1997) 335-351.

[35] M. Arroyo, M. Ortiz, Local maximum-entropy approximation schemes: a seamless bridge between finite elements and meshfree methods., International Journal for Numerical Methods in Engineering 65 (13) (2006) 2167-2202.

[36] V. V. Belikov, V. D. Ivanov, V. K. Kontorovich, S. A. Korytnik, A. Y. Semenov, The non-Sibsonian interpolation: A new method of interpolation of the values of a function on an arbitrary set of points., Computational Mathematics and Mathematical Physics 37 (1) (1997) 9-15.

[37] M. S. Floater, K. Hormann, G. Kòs, A general construction of barycentric coordinates over convex polygons., Advances in Computational Mathematics 24 (1-4) (2004) 311-331.

[38] H. Hiyoshi, K. Sugihara, Two generalizations of an interpolant based on Voronoi diagrams., International Journal of Shape Modeling 5 (2) (1999) 219-231. 
[39] P. Joshi, M. Meyer, T. DeRose, B. Green, T. Sanocki, Harmonic coordinates for character articulation, in: ACM Transactions on Graphics (TOG), Vol. 26, ACM, 2007, p. 71.

[40] E. A. Malsch, J. J. Lin, G. Dasgupta, Smooth two dimensional interpolants: a recipe for all polygons, Journal of Graphics Tools 10 (2005) 2.

[41] M. Meyer, A. Barr, H. Lee, M. Desbrun, Generalized barycentric coordinates on irregular polygons, J Graphics Tools 7 (2002) 13-22.

[42] N. Sukumar, Construction of polygonal interpolants: a maximum entropy approach., International Journal of Numerical Methods in Engineering 61 (12) (2004) 2159-2181.

[43] M. S. Floater, Mean value coordinates., Computer Aided Geometric Design 20 (1) (2003) 19-27.

[44] M. Floater, Wachspress and mean value coordinates, in: Approximation Theory XIV: San Antonio 2013, Springer, 2014, pp. 81-102.

[45] M. Floater, Generalized barycentric coordinates and applications, Acta Numerica 24 (2015) 161-214.

[46] A. Rand, A. Gillette, C. Bajaj, Interpolation error estimates for mean value coordinates over convex polygons, Advances in computational mathematics 39 (2) (2013) 327-347.

[47] K. Hormann, M. Floater, Mean value coordinates for arbitrary planar polygons, ACM Transactions on Graphics (TOG) 25 (4) (2006) 1424-1441.

[48] M. Crouzeix, P. Raviart, Conforming and nonconforming finite element methods for solving the stationary stokes equation, Rev Fr Autom Inf Rech Oper 7 (1973) 33-76.

[49] C. Taylor, P. Hood, A numerical solution of the navier-stokes equations using the finite element technique, Computers \& Fluids 1 (1) (1973) 73-100.

[50] N. Sukumar, A. Tabarraei, Conforming polygonal finite elements., International Journal of Numerical Methods in Engineering 61 (12) (2004) 2045-2066.

[51] D. Dunavant, High degree efficient symmetrical gaussian quadrature rules for the triangle, International journal for numerical methods in engineering 21 (6) (1985) 1129-1148.

[52] S. E. Mousavi, H. Xiao, N. Sukumar, Generalized Gaussian quadrature rules on arbitrary polygons., International Journal for Numerical Methods in Engineering 82 (1) (2010) 99113. 
[53] S. Mousavi, N. Sukumar, Numerical integration of polynomials and discontinuous functions on irregular convex polygons and polyhedrons, Computational Mechanics 47 (5) (2011) $535-554$.

[54] E. Chin, J. Lasserre, N. Sukumar, Numerical integration of homogeneous functions on convex and nonconvex polygons and polyhedra, Compuational Mechanics 56 (6) (2015) 967-981.

[55] G. Strang, G. Fix, An Analysis of the Finite Element Method, 2nd Edition, WellesleyCambridge, 2008.

[56] P. G. Ciarlet, The Finite Element Method for Elliptic Problems, 2nd Edition, Society for Industrial and Applied Mathematics, 2002.

[57] L. B. Da Veiga, F. Brezzi, L. D. Marini, Virtual elements for linear elasticity problems, SIAM Journal on Numerical Analysis 51 (2) (2013) 794-812.

[58] C. Talischi, G. H. Paulino, A. Pereira, I. F. M. Menezes, Polymesher: A general-purpose mesh generator for polygonal elements written in Matlab., Structural and Multidisciplinary Optimization 45 (3) (2012) 309-328.

[59] M. Biot, Surface instability of rubber in compression, Applied Scientific Research, Section A 12 (2) (1963) 168-182.

[60] H. Chi, O. Lopez-Pamies, G. Paulino, A variational formulation with rigid-body constraints for finite elasticity: Theory, finite element implementation, and applications, Compuational MechanicsSubmitted.

[61] J. Leblanc, Filled Polymers: Science and Industrial Applications, CRC Press, 2010.

[62] O. Lopez-Pamies, T. Goudarzi, K. Danas, The nonlinear elastic response of suspensions of rigid inclusions in rubber: II - a simple explicit approximation for finite-concentration suspensions, Journal of the Mechanics and Physics of Solids 61 (2013) 19-37.

[63] T. Goudarzi, D. Spring, G. Paulino, O. Lopez-Pamies, Filled elastomers: A theory of filler reinforcement based on hydrodynamic and interphasial effects, Journal of the Mechanics and Physics of Solids 80 (2015) 37-67.

[64] O. Lopez-Pamies, An exact result for the macroscopic response of particle-reinforced neohookean solids, Journal of Applied Mechanics 77 (2) (2010) 021016. 
[65] J. Moraleda, J. Segurado, J. LLorca, Finite deformation of incompressible fiber-reinforced elastomers: a computational micromechanics approach, Journal of the Mechanics and Physics of Solids 57 (9) (2009) 1596-1613.

[66] A. Gent, Cavitation in rubber: a cautionary tale, Rubber Chemistry and Technology 63 (3) (1990) 49-53.

[67] K. Cho, A. N. Gent, Cavitation in model elastomeric composites, Journal of materials science 23 (1) (1988) 141-144.

[68] V. Lefevre, K. Ravi-Chandar, O. Lopez-Pamies, Cavitation in rubber: an elastic instability or a fracture phenomenon?, International Journal of Fracture (2014) 1-23.

[69] O. Lopez-Pamies, A new $I_{1}$-based hyperelastic model for rubber elastic materials, Comptes Rendus Mecanique 338 (1) (2010) 3-11.

[70] J. M. Ball, Discontinuous equilibrium solutions and cavitation in nonlinear elasticity, Philosophical Transactions of the Royal Society of London. Series A, Mathematical and Physical Sciences 306 (1496) (1982) 557-611.

[71] X. Xu, D. Henao, An efficient numerical method for cavitation in nonlinear elasticity, Mathematical Models and Methods in Applied Sciences 21 (08) (2011) 1733-1760.

[72] T. Nakamura, O. Lopez-Pamies, A finite element approach to study cavitation instabilities in non-linear elastic solids under general loading conditions, International Journal of NonLinear Mechanics 47 (2) (2012) 331-340.

[73] O. Lopez-Pamies, T. Nakamura, M. I. Idiart, Cavitation in elastomeric solids: I - a defect-growth theory, Journal of the Mechanics and Physics of Solids 59 (2011) 1464-1487.

[74] O. Lopez-Pamies, T. Nakamura, M. I. Idiart, Cavitation in elastomeric solids: II — onsetof-cavitation surfaces for Neo-Hookean materials, Journal of the Mechanics and Physics of Solids 59 (2011) 1488-1505.

[75] O. Lopez-Pamies, Onset of cavitation in compressible, isotropic, hyperelastic solids, Journal of elasticity 94 (2) (2009) 115-145. 